\title{
Aldgamycins J-O, 16-Membered Macrolides with a Branched Octose Unit from Streptomycetes sp. and Their Antibacterial Activities
}

Chuan-Xi Wang, ${ }^{\dagger, \perp}$ Rong Ding, ${ }^{\dagger, \perp}$ Shu-Tai Jiang, ${ }^{\dagger}$ Jin-Shan Tang, ${ }^{\dagger}$ Dan Hu, ${ }^{\dagger}$ Guo-Dong Chen, ${ }^{\dagger}$ Feng Lin,${ }^{\S}$ Kui Hong, ${ }^{* \star}$ Xin-Sheng Yao, ${ }^{* \dagger}$ Hao Gao ${ }^{*}$,

${ }^{\dagger}$ Institute of Traditional Chinese Medicine and Natural Products, College of Pharmacy, Jinan University, Guangzhou 510632, People’s Republic of China

${ }^{\ddagger}$ Key Laboratory of Combinatorial Biosynthesis and Drug Discovery (Wuhan University), Ministry of Education, and Wuhan University School of Pharmaceutical Sciences, Wuhan 430071, People's Republic of China

${ }^{\S}$ Fujian key Laboratory of Screening for Novel Microbial Products, Fujian Institute of Microbiology, Fuzhou 350007, People's Republic of China

*To whom correspondence should be addressed. Telephone/Fax: +86-20-85228369. E-mail: (H.G.) tghao@jnu.edu.cn; (X.Y.) tyaoxs@jnu.edu.cn; (K.H.) kuihong31@ whu.edu.cn. 


\section{List of Supporting Information}

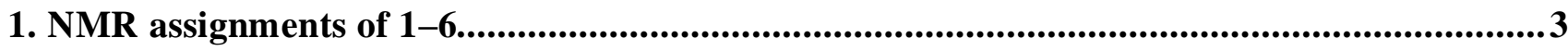

Table S1. NMR (400 MHz, $\left.\mathrm{CDCl}_{3}\right)$ assignments for 1 ......................................................... 3

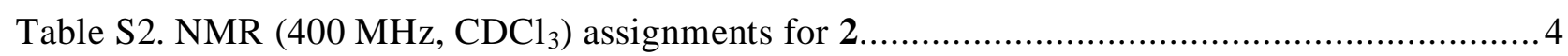

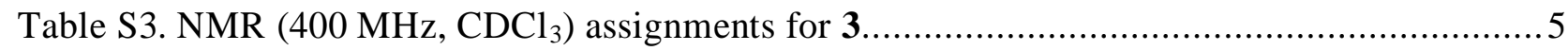

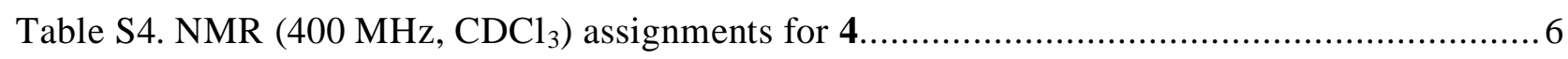

Table S5. NMR (400 MHz, $\mathrm{CDCl}_{3}$ ) assignments for 5 ........................................................

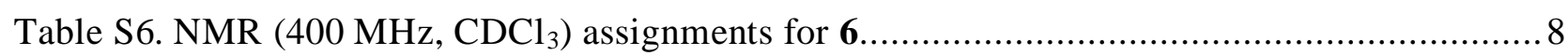

2. Acid hydrolysis of aldgamycin $J(1)$................................................................................................9

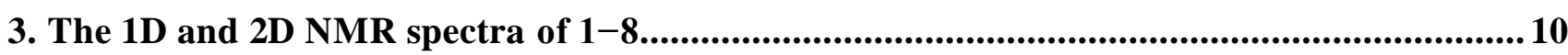

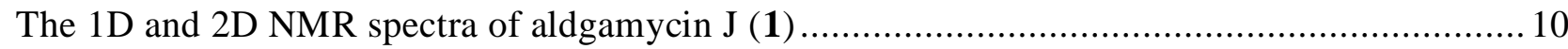

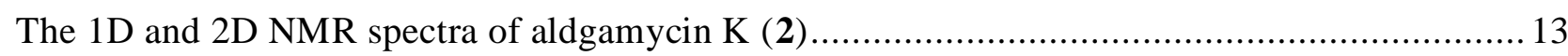

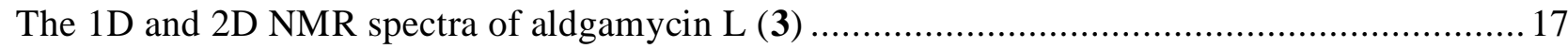

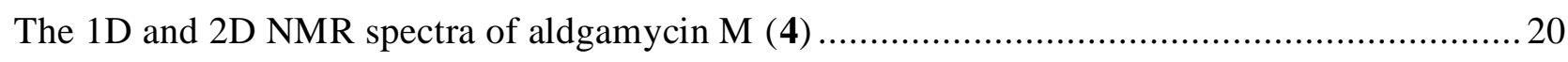

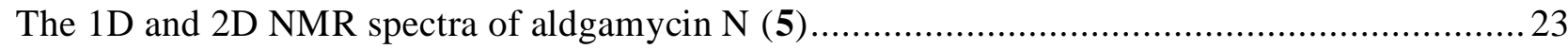

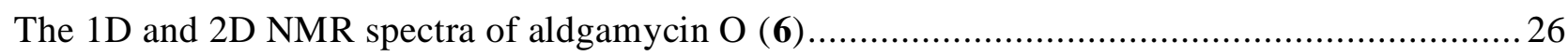

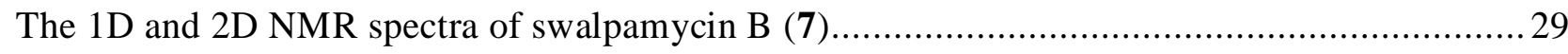

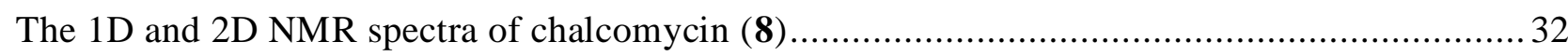




\section{NMR assignments of 1-6}

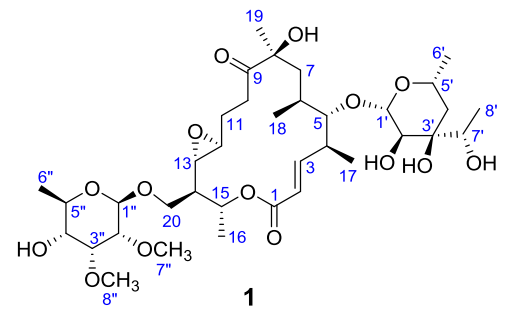

Table S1. NMR (400 MHz, $\left.\mathrm{CDCl}_{3}\right)$ assignments for $\mathbf{1}$.

\begin{tabular}{|c|c|c|c|c|c|}
\hline Position & $\delta_{\mathrm{C}}$, mult. & $\delta_{\mathrm{H}}(J \text { in } \mathrm{Hz})^{*}$ & ${ }^{1} \mathrm{H},{ }^{1} \mathrm{H}-\mathrm{COSY}$ & HMBC & ROESY \\
\hline \multicolumn{6}{|c|}{ aglycone } \\
\hline 1 & $165.5, \mathrm{C}$ & - & & & \\
\hline 2 & $121.2, \mathrm{CH}$ & $5.86 \mathrm{~d}(15.5)$ & 3 & 1,4 & 4 \\
\hline 3 & $151.3, \mathrm{CH}$ & $6.74 \mathrm{dd}(15.5,10.6)$ & 2,4 & 1 & $5,6,17$ \\
\hline 4 & $41.8, \mathrm{CH}$ & 2.71 & $3,5,17$ & 5 & $2,6,7 \mathrm{a}, 7 \mathrm{~b}$ \\
\hline 5 & $86.7, \mathrm{CH}$ & 3.32 br. d (9.7) & 4,6 & $4,7,18,1^{\prime}$ & $3,6,17,18,1^{\prime}$ \\
\hline 6 & $34.2, \mathrm{CH}$ & 1.35 & $5,7,18$ & 7,8 & $3,4,5,7 \mathrm{a}, 10 \mathrm{a}$ \\
\hline \multirow[t]{2}{*}{7} & $37.0, \mathrm{CH}_{2}$ & $1.89, \mathrm{Ha}$ & 6 & 8,9 & $4,6,10 \mathrm{a}, 19$ \\
\hline & & $1.86, \mathrm{Hb}$ & 6 & 8,9 & $4,18,19$ \\
\hline 8 & 79.6, C & - & & & \\
\hline 9 & $212.5, \mathrm{C}$ & - & & & \\
\hline \multirow[t]{2}{*}{10} & $32.6, \mathrm{CH}_{2}$ & 2.70, Нa & $10 b, 11 a, 11 b$ & 11 & $6,7 \mathrm{a}$ \\
\hline & & $2.16, \mathrm{Hb}$ & $10 \mathrm{a}, 11 \mathrm{a}, 11 \mathrm{~b}$ & & \\
\hline \multirow[t]{2}{*}{11} & 27.2, $\mathrm{CH}_{2}$ & $2.00, \mathrm{Ha}$ & $10 \mathrm{a}, 10 \mathrm{~b}, 11 \mathrm{~b}, 12$ & 10,13 & \\
\hline & & $1.55, \mathrm{Hb}$ & $10 \mathrm{a}, 10 \mathrm{~b}, 11 \mathrm{a}, 12$ & 12 & 13 \\
\hline 12 & $59.3, \mathrm{CH}$ & 2.73 & $11 \mathrm{a}, 11 \mathrm{~b}, 13$ & 11 & 14,15 \\
\hline 13 & $58.0, \mathrm{CH}$ & $2.82 \mathrm{dd}(8.9,2.0)$ & 12,14 & 14 & $11 b, 20 a, 15$ \\
\hline 14 & $48.6, \mathrm{CH}$ & 1.37 & $13,15,20 a, 20 b$ & $13,15,16$ & 12 \\
\hline 15 & $69.9, \mathrm{CH}$ & 5.32 dq. $(10.2,6.3)$ & 14,16 & 1 & $12,13,20 \mathrm{~b}$ \\
\hline 16 & $18.6, \mathrm{CH}_{3}$ & $1.35 \mathrm{~d}(6.3)$ & 15 & 14,15 & $20 \mathrm{a}$ \\
\hline 17 & $20.7, \mathrm{CH}_{3}$ & $1.20 \mathrm{~d}(6.6)$ & 4 & $3,4,5$ & $3,5,1^{\prime}$ \\
\hline 18 & $18.7, \mathrm{CH}_{3}$ & $0.99 \mathrm{~d}(6.8)$ & 6 & $5,6,7$ & $5,7 \mathrm{~b}$ \\
\hline 19 & $28.2, \mathrm{CH}_{3}$ & $1.38 \mathrm{~s}$ & & $7,8,9$ & $7 \mathrm{a}, 7 \mathrm{~b}$ \\
\hline \multirow[t]{2}{*}{20} & $67.2, \mathrm{CH}_{2}$ & $4.15 \mathrm{dd}(10.1,3.5), \mathrm{Ha}$ & $14,20 \mathrm{~b}$ & 15 & $13,16,1 "$ \\
\hline & & $3.63 \mathrm{dd}(10.1,3.5), \mathrm{Hb}$ & $14,20 \mathrm{a}$ & 13,15 & 15 \\
\hline \multicolumn{6}{|c|}{$\beta$-D-decarboxylated aldgarose } \\
\hline $1^{\prime}$ & $101.3, \mathrm{CH}$ & $4.63 \mathrm{~d}(7.6)$ & $2^{\prime}$ & 5 & $5,17,5^{\prime}$ \\
\hline $2^{\prime}$ & $72.6, \mathrm{CH}$ & $3.62 \mathrm{~m}^{c}$ & $1^{\prime}$ & $1^{\prime}$ & $4^{\prime} \mathrm{b}, 8^{\prime}$ \\
\hline $3^{\prime}$ & 73.9, C & - & & & \\
\hline \multirow[t]{2}{*}{$4^{\prime}$} & $39.5, \mathrm{CH}_{2}$ & 1.50 br.d (13.7), Ha & $4^{\prime} \mathrm{b}, 5^{\prime}$ & $2^{\prime}, 3^{\prime}, 7^{\prime}$ & $7^{\prime}$ \\
\hline & & $1.35, \mathrm{Hb}$ & $4^{\prime} \mathrm{a}, 5^{\prime}$ & $3^{\prime}, 5^{\prime}, 6^{\prime}, 7^{\prime}$ & $2^{\prime}$ \\
\hline $5^{\prime}$ & $66.8, \mathrm{CH}$ & 3.93 & $6^{\prime}, 4^{\prime} \mathrm{a}, 4^{\prime} \mathrm{b}$ & & $1^{\prime}$ \\
\hline $6^{\prime}$ & $20.8, \mathrm{CH}_{3}$ & $1.16 \mathrm{~d}(6.3)$ & $5^{\prime}$ & $4^{\prime}, 5^{\prime}$ & \\
\hline $7^{\prime}$ & $73.9, \mathrm{CH}$ & $3.66 \mathrm{q}(6.6)$ & $8^{\prime}$ & $3^{\prime}$ & $4^{\prime} \mathrm{a}$ \\
\hline $8^{\prime}$ & $18.2, \mathrm{CH}_{3}$ & $1.29 \mathrm{~d}(6.6)$ & $7^{\prime}$ & $3^{\prime}, 7^{\prime}$ & $2^{\prime}$ \\
\hline \multicolumn{6}{|c|}{$\beta$-D-mycinose } \\
\hline $1 "$ & $100.9, \mathrm{CH}$ & $4.57 \mathrm{~d}(7.7)$ & $2^{\prime \prime}$ & 20 & 20a, 5", 7" \\
\hline $2^{\prime \prime}$ & $81.9, \mathrm{CH}$ & $3.08 \mathrm{dd}(7.7,2.8)$ & 1", 3" & 1", 7" & 4", 7" \\
\hline $3^{\prime \prime}$ & $79.6, \mathrm{CH}$ & $3.77 \mathrm{t}(2.8)$ & 2", 4" & 1", 4", 5", 8" & \\
\hline $4 "$ & $72.7, \mathrm{CH}$ & $3.20 \mathrm{dd}(9.0,2.8)$ & 3", 5" & & $2 ", 6 "$ \\
\hline $5^{\prime \prime}$ & $70.8, \mathrm{CH}$ & 3.54 & 4", 6" & $3^{\prime \prime}$ & $1 "$ \\
\hline $6^{\prime \prime}$ & $17.8, \mathrm{CH}_{3}$ & $1.26 \mathrm{~d}(6.2)$ & $5^{\prime \prime}$ & $4 ", 5 "$ & $4 "$ \\
\hline $7 "$ & $59.6, \mathrm{CH}_{3}$ & $3.56 \mathrm{~s}$ & & $2^{\prime \prime}$ & 1", 2" \\
\hline $8^{\prime \prime}$ & $61.7, \mathrm{CH}_{3}$ & $3.62 \mathrm{~s}$ & & $3^{\prime \prime}$ & \\
\hline
\end{tabular}

* Indiscernible signals owing to overlapping or having complex multiplicity are reported without designating multiplicity. 


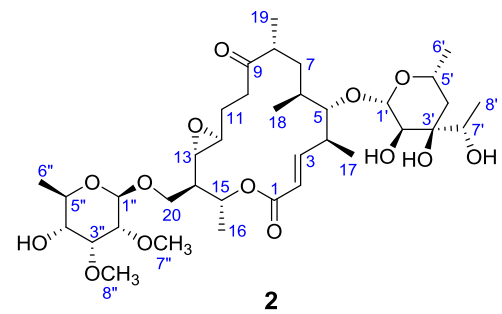

Table S2. NMR (400 MHz, $\mathrm{CDCl}_{3}$ ) assignments for 2.

\begin{tabular}{|c|c|c|c|c|c|}
\hline Position & $\delta_{\mathrm{C}}$, mult. & $\delta_{\mathrm{H}}(J \text { in } \mathrm{Hz})^{*}$ & ${ }^{1} \mathrm{H},{ }^{1} \mathrm{H}-\mathrm{COSY}$ & HMBC & ROESY \\
\hline \multicolumn{6}{|c|}{ aglycone } \\
\hline 1 & $165.6, \mathrm{C}$ & - & & & \\
\hline 2 & $121.2, \mathrm{CH}$ & $5.86 \mathrm{~d}(15.5)$ & 3 & 1,4 & 4 \\
\hline 3 & $151.1, \mathrm{CH}$ & $6.75 \mathrm{dd}(15.5,10.5)$ & 2,4 & 1 & $5,6,17$ \\
\hline 4 & $41.3, \mathrm{CH}$ & 2.74 & $3,5,17$ & & $2,6,7 \mathrm{a}$ \\
\hline 5 & $85.5, \mathrm{CH}$ & 3.42 br d (10.0) & 4,6 & $7,17,18,1^{\prime}$ & $3,6,7 \mathrm{~b}, 8,17,18,1^{\prime}$ \\
\hline 6 & $34.8, \mathrm{CH}$ & 1.41 & $5,7 \mathrm{a}, 7 \mathrm{~b}, 18$ & 18 & $3,4,5,7 \mathrm{a}, 8,10 \mathrm{~b}$ \\
\hline \multirow[t]{2}{*}{7} & $32.5, \mathrm{CH}_{2}$ & $1.71 \mathrm{ddd}(13.8,9.7,3.2), \mathrm{Ha}$ & $6,7 b, 8$ & & $4,6,10 \mathrm{a}, 19$ \\
\hline & & $1.38, \mathrm{Hb}$ & $6,7 a, 8$ & & 5,18 \\
\hline 8 & $45.5, \mathrm{CH}$ & 2.52 & $7 a, 7 b, 19$ & & $5,6,18,19$ \\
\hline 9 & $213.3, \mathrm{C}$ & - & & & \\
\hline \multirow[t]{2}{*}{10} & $33.4, \mathrm{CH}_{2}$ & $2.54, \mathrm{Ha}$ & $10 \mathrm{~b}, 11 \mathrm{a}, 11 \mathrm{~b}$ & & $7 \mathrm{a}, 12$ \\
\hline & & $2.06, \mathrm{Hb}$ & $10 a, 11 a, 11 b$ & & 6 \\
\hline \multirow[t]{2}{*}{11} & $26.8, \mathrm{CH}_{2}$ & $2.02, \mathrm{Ha}$ & $10 \mathrm{a}, 10 \mathrm{~b}, 11 \mathrm{~b}, 12$ & & \\
\hline & & $1.43, \mathrm{Hb}$ & $10 \mathrm{a}, 10 \mathrm{~b}, 11 \mathrm{a}, 12$ & & 13 \\
\hline 12 & $59.5, \mathrm{CH}$ & $2.83 \mathrm{dd}(8.2,2.5)$ & $11 \mathrm{a}, 11 \mathrm{~b}, 13$ & 11 & 10a, 14 \\
\hline 13 & $58.0, \mathrm{CH}$ & $2.73 \mathrm{dd}(8.2,2.2)$ & 12,14 & 14 & $15,20 \mathrm{a}, 11 \mathrm{~b}$ \\
\hline 14 & $48.5, \mathrm{CH}$ & 1.36 & $13,15,20 \mathrm{a}, 20 \mathrm{~b}$ & 15,16 & 12 \\
\hline 15 & $69.8, \mathrm{CH}$ & 5.32 dq. $(9.6,6.3)$ & 14,16 & & $13,20 b$ \\
\hline 16 & $18.5, \mathrm{CH}_{3}$ & $1.35 \mathrm{~d}(6.3)$ & 15 & 14,15 & $20 \mathrm{a}, 20 \mathrm{~b}$ \\
\hline 17 & $18.2, \mathrm{CH}_{3}$ & $1.21 \mathrm{~d}(6.6)$ & 4 & $3,4,5$ & $3,5,1^{\prime}$ \\
\hline 18 & $17.0, \mathrm{CH}_{3}$ & $0.98 \mathrm{~d}(6.4)$ & 6 & $5,6,7$ & $5,7 \mathrm{~b}, 8$ \\
\hline 19 & $17.8, \mathrm{CH}_{3}$ & $1.11 \mathrm{~d}(6.9)$ & 8 & $7,8,9$ & $7 \mathrm{a}, 8$ \\
\hline \multirow[t]{2}{*}{20} & $67.3, \mathrm{CH}_{2}$ & $4.12 \mathrm{dd}(10.2,3.6), \mathrm{Ha}$ & $14,20 \mathrm{~b}$ & 15 & $13,16,1 "$ \\
\hline & & $3.62, \mathrm{Hb}$ & $14,20 \mathrm{a}$ & 13 & 15,16 \\
\hline \multicolumn{6}{|c|}{$\beta$-D-decarboxylated aldgarose } \\
\hline $1^{\prime}$ & 101.7, $\mathrm{CH}$ & $4.66 \mathrm{~d}(7.7)$ & $2^{\prime}$ & 5 & $5,17,5^{\prime}$ \\
\hline $2^{\prime}$ & $72.7, \mathrm{CH}$ & 3.61 & $1^{\prime}$ & $1^{\prime}$ & $4^{\prime} \mathrm{b}, 8^{\prime}$ \\
\hline $3^{\prime}$ & 73.9, C & - & & & \\
\hline \multirow[t]{2}{*}{$4^{\prime}$} & $39.4, \mathrm{CH}_{2}$ & $1.51, \mathrm{Ha}$ & $4^{\prime} \mathrm{b}, 5^{\prime}$ & $2^{\prime}, 3^{\prime}, 7^{\prime}$ & $7^{\prime}$ \\
\hline & & $1.38, \mathrm{Hb}$ & $4^{\prime} \mathrm{a}, 5^{\prime}$ & & $2^{\prime}$ \\
\hline $5^{\prime}$ & $67.0, \mathrm{CH}$ & 3.96 & $6^{\prime}, 4^{\prime} \mathrm{a}, 4^{\prime} \mathrm{b}$ & & $1^{\prime}$ \\
\hline $6^{\prime}$ & $20.8, \mathrm{CH}_{3}$ & $1.18 \mathrm{~d}(6.1)$ & $5^{\prime}$ & $4^{\prime}, 5^{\prime}$ & \\
\hline $7^{\prime}$ & $73.9, \mathrm{CH}$ & $3.66 \mathrm{q}(6.6)$ & $8^{\prime}$ & & 4'a \\
\hline $8^{\prime}$ & $18.3, \mathrm{CH}_{3}$ & $1.29 \mathrm{~d}(6.6)$ & $7^{\prime}$ & $3^{\prime}, 7^{\prime}$ & $2^{\prime}$ \\
\hline \multicolumn{6}{|c|}{$\beta$-D-mycinose } \\
\hline $1 "$ & $100.8, \mathrm{CH}$ & $4.56 \mathrm{~d}(7.7)$ & $2^{\prime \prime}$ & 20 & 20a, 5", 7" \\
\hline $2^{\prime \prime}$ & $81.9, \mathrm{CH}$ & $3.08 \mathrm{dd}(7.7,3.0)$ & 1", 3" & 1", 7" & 7", \\
\hline $3^{\prime \prime}$ & $79.6, \mathrm{CH}$ & $3.76 \mathrm{t}(3.0)$ & 2", 4" & 1", 4", 5", 8" & \\
\hline $4 "$ & $72.7, \mathrm{CH}$ & $3.19 \mathrm{dd}(9.1,3.0)$ & 3", 5" & & $6^{\prime \prime}$ \\
\hline $5^{\prime \prime}$ & $70.8, \mathrm{CH}$ & 3.52 & 4", 6" & 3" & $1 "$ \\
\hline $6^{\prime \prime}$ & $17.8, \mathrm{CH}_{3}$ & $1.25 \mathrm{~d}(6.2)$ & $5^{\prime \prime}$ & 4", 5" & $4 "$ \\
\hline $7 "$ & $59.6, \mathrm{CH}_{3}$ & $3.55 \mathrm{~s}$ & & $2^{\prime \prime}$ & 1", 2" \\
\hline $8^{\prime \prime}$ & $61.6, \mathrm{CH}_{3}$ & $3.61 \mathrm{~s}$ & & 3" & \\
\hline
\end{tabular}

${ }^{*}$ Indiscernible signals owing to overlapping or having complex multiplicity are reported without designating multiplicity. 


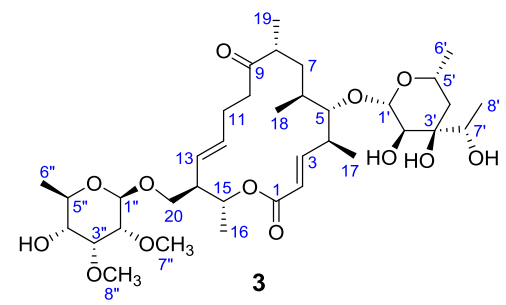

Table S3. NMR (400 MHz, $\left.\mathrm{CDCl}_{3}\right)$ assignments for 3.

\begin{tabular}{|c|c|c|c|c|c|}
\hline Position & $\delta_{\mathrm{C}}$, mult. & $\delta_{\mathrm{H}}(J \text { in } \mathrm{Hz})^{*}$ & ${ }^{1} \mathrm{H},{ }^{1} \mathrm{H}-\mathrm{COSY}$ & HMBC & ROESY \\
\hline \multicolumn{6}{|c|}{ aglycone } \\
\hline 1 & $165.6, \mathrm{C}$ & - & & & \\
\hline 2 & 121.7, $\mathrm{CH}$ & $5.80 \mathrm{~d}(15.5)$ & 3 & 1,4 & 4,17 \\
\hline 3 & $150.3, \mathrm{CH}$ & $6.71 \mathrm{dd}(15.5,9.9)$ & 2,4 & 1 & 5,617 \\
\hline 4 & $40.7, \mathrm{CH}$ & 2.72 & $3,5,17$ & $2,3,5$ & $2,6,7 \mathrm{a}$ \\
\hline 5 & $86.2, \mathrm{CH}$ & 3.39 br. d (9.6) & 4,6 & $4,7,17,18,1^{\prime}$ & $3,6,8,17,18,1^{\prime}$ \\
\hline 6 & $34.7, \mathrm{CH}$ & 1.54 & $5,7 \mathrm{a}, 7 \mathrm{~b}, 18$ & & $3,4,5,8$ \\
\hline 7 & 33.0, $\mathrm{CH}_{2}$ & $\begin{array}{c}1.68 \text { ddd }(13.6,9.4,3.5), \mathrm{Ha} \\
1.33, \mathrm{Hb}\end{array}$ & $\begin{array}{c}6,8,7 \mathrm{~b} \\
6,7 \mathrm{a}\end{array}$ & 6,18 & $\begin{array}{c}4,10 \mathrm{a}, 19 \\
18\end{array}$ \\
\hline 8 & $44.8, \mathrm{CH}$ & 2.54 & $7 \mathrm{a}, 19$ & 9 & $5,6,18,19$ \\
\hline 9 & $213.6, \mathrm{C}$ & - & & & \\
\hline \multirow[t]{2}{*}{10} & $38.9, \mathrm{CH}_{2}$ & $2.51, \mathrm{Ha}$ & $10 \mathrm{~b}, 11 \mathrm{a}, 11 \mathrm{~b}$ & 9,11 & $7 \mathrm{a}, 12,13$ \\
\hline & & $2.21, \mathrm{Hb}$ & $10 \mathrm{a}, 11 \mathrm{a}, 11 \mathrm{~b}$ & 9,11 & \\
\hline \multirow[t]{2}{*}{11} & 26.1, $\mathrm{CH}_{2}$ & $2.19, \mathrm{Ha}$ & $10 \mathrm{a}, 10 \mathrm{~b}, 12$ & & 13 \\
\hline & & $2.16, \mathrm{Hb}$ & $10 \mathrm{a}, 10 \mathrm{~b}, 12$ & $9,10,12$ & 13 \\
\hline 12 & $132.7, \mathrm{CH}$ & 5.52 ddd $(15.3,9.4,3.5)$ & $11 \mathrm{a}, 11 \mathrm{~b}, 13$ & 11 & 10a, 14 \\
\hline 13 & $128.6, \mathrm{CH}$ & $5.30 \mathrm{dd}(15.3,9.2)$ & 12,14 & $11,12,15$ & $10 \mathrm{a}, 11 \mathrm{a}, 11 \mathrm{~b}, 14,15,20 \mathrm{~b}$ \\
\hline 14 & $50.2, \mathrm{CH}$ & 2.36 & $13,15,20 \mathrm{a}, 20 \mathrm{~b}$ & 15 & $12,13,16$ \\
\hline 15 & $69.9, \mathrm{CH}$ & 5.07 dq. $(9.0,6.0)$ & 14,16 & 1,13 & $13,20 \mathrm{a}, 20 \mathrm{~b}$ \\
\hline 16 & $18.2, \mathrm{CH}_{3}$ & $1.29 \mathrm{~d}(6.0)$ & 15 & 14,15 & $14,20 \mathrm{a}, 20 \mathrm{~b}$ \\
\hline 17 & $18.8, \mathrm{CH}_{3}$ & $1.19 \mathrm{~d}(6.6)$ & 4 & $3,4,5$ & $2,3,5,1^{\prime}$ \\
\hline 18 & $17.1, \mathrm{CH}_{3}$ & $0.98 \mathrm{~d}(6.7)$ & 6 & $5,6,7$ & $5,7 \mathrm{~b}, 8$ \\
\hline 19 & $18.6, \mathrm{CH}_{3}$ & $1.10 \mathrm{~d}(6.9)$ & 8 & $7,8,9$ & $7 \mathrm{a}, 8$ \\
\hline \multirow[t]{3}{*}{20} & $70.0, \mathrm{CH}_{2}$ & $3.93 \mathrm{dd}(9.6,5.0), \mathrm{Ha}$ & $14,20 \mathrm{~b}$ & 15 & $15,16,1 "$ \\
\hline & & $3.45 \mathrm{dd}(9.6,5.0), \mathrm{Hb}$ & $14,20 \mathrm{a}$ & $13,14,15$ & $13,15,16$ \\
\hline & \multicolumn{5}{|c|}{$\beta$-D-decarboxylated aldgarose } \\
\hline $1^{\prime}$ & $101.7, \mathrm{CH}$ & $4.66 \mathrm{~d}(7.6)$ & $2^{\prime}$ & 5 & $5,17,5^{\prime}$ \\
\hline $2^{\prime}$ & $72.6, \mathrm{CH}$ & 3.63 & $1^{\prime}$ & $1^{\prime}$ & $4^{\prime} b, 8^{\prime}$ \\
\hline $3^{\prime}$ & 73.9, C & - & & & \\
\hline \multirow[t]{2}{*}{$4^{\prime}$} & $39.4, \mathrm{CH}_{2}$ & 1.53 br. d (13.6), Ha & $4^{\prime} \mathrm{b}, 5^{\prime}$ & $2^{\prime}, 3^{\prime}, 7^{\prime}$ & $7^{\prime}$ \\
\hline & & $1.40, \mathrm{Hb}$ & $4^{\prime} \mathrm{a}, 5^{\prime}$ & & $2^{\prime}$ \\
\hline $5^{\prime}$ & $67.0, \mathrm{CH}$ & 3.97 & $6^{\prime}, 4^{\prime} \mathrm{a}, 4^{\prime} \mathrm{b}$ & & $1^{\prime}$ \\
\hline $6^{\prime}$ & $20.8, \mathrm{CH}_{3}$ & $1.18 \mathrm{~d}(6.1)$ & $5^{\prime}$ & $4^{\prime}, 5^{\prime}$ & \\
\hline $7^{\prime}$ & 73.7, $\mathrm{CH}$ & $3.66 \mathrm{q}(6.1)$ & $8^{\prime}$ & $3^{\prime}$ & 4'a \\
\hline $8^{\prime}$ & $18.2, \mathrm{CH}_{3}$ & $1.28 \mathrm{~d}(6.1)$ & $7^{\prime}$ & $3^{\prime}, 7^{\prime}$ & $2^{\prime}$ \\
\hline \multicolumn{6}{|c|}{$\beta$-D-mycinose } \\
\hline $1 "$ & 101.0, CH & $4.56 \mathrm{~d}(7.7)$ & $2^{\prime \prime}$ & 20 & 20a, 5", 7" \\
\hline $2^{\prime \prime}$ & $81.9, \mathrm{CH}$ & $3.04 \mathrm{dd}(7.7,2.8)$ & 1", 3" & 1", 7" & 4", 7" \\
\hline 3" & 79.9, CH & $3.74 \mathrm{t}(2.8)$ & 2", 4" & 1", 2", 4", 5", 8" & \\
\hline 4" & 72.7, $\mathrm{CH}$ & $3.19 \mathrm{dd}(9.4,2.8)$ & 3", 5" & & 2", 6" \\
\hline $5^{\prime \prime}$ & $70.7, \mathrm{CH}$ & 3.51 & 4", 6" & $3 "$ & $1 "$ \\
\hline $6^{\prime \prime}$ & $17.8, \mathrm{CH}_{3}$ & $1.25 \mathrm{~d}(6.2)$ & $5^{\prime \prime}$ & 4", 5" & $4 "$ \\
\hline $7 "$ & $59.8, \mathrm{CH}_{3}$ & $3.53 \mathrm{~s}$ & & $2^{\prime \prime}$ & 1", 2" \\
\hline $8^{\prime \prime}$ & $61.7, \mathrm{CH}_{3}$ & $3.61 \mathrm{~s}$ & & $3 "$ & \\
\hline
\end{tabular}

${ }^{*}$ Indiscernible signals owing to overlapping or having complex multiplicity are reported without designating multiplicity. 


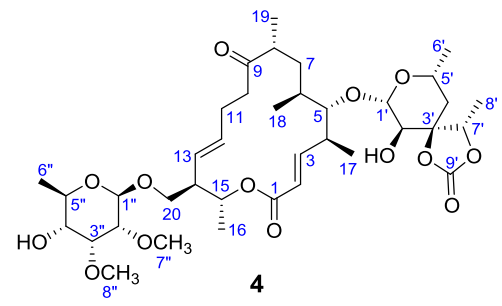

Table S4. NMR (400 MHz, $\left.\mathrm{CDCl}_{3}\right)$ assignments for 4.

\begin{tabular}{|c|c|c|c|c|c|}
\hline Position & $\delta_{\mathrm{C}}$, mult. & $\delta_{\mathrm{H}}(\mathrm{J} \text { in } \mathrm{Hz})^{*}$ & ${ }^{1} \mathrm{H},{ }^{1} \mathrm{H}-\mathrm{COS} \mathrm{Y}$ & HMBC & ROESY \\
\hline \multicolumn{6}{|c|}{ aglycone } \\
\hline 1 & $165.4, \mathrm{C}$ & - & & & \\
\hline 2 & $121.9, \mathrm{CH}$ & $5.80 \mathrm{~d}(15.5)$ & 3 & 1,4 & 4,17 \\
\hline 3 & $149.7, \mathrm{CH}$ & $6.69 \mathrm{dd}(15.5,9.6)$ & 2,4 & 1 & 5,17 \\
\hline 4 & $40.6, \mathrm{CH}$ & 2.72 & $3,5,17$ & $2,3,5,17$ & $2,7 \mathrm{a}$ \\
\hline 5 & $86.3, \mathrm{CH}$ & 3.41 br. d (10.1) & 4,6 & $4,7,17,18,1^{\prime}$ & $3,17,18,1^{\prime}$ \\
\hline 6 & $34.5, \mathrm{CH}$ & 1.52 & $5,7 \mathrm{a}, 7 \mathrm{~b}, 18$ & & \\
\hline 7 & $32.9, \mathrm{CH}_{2}$ & $\begin{array}{c}1.68 \text { ddd }(14.1,10.0,4.2), \mathrm{Ha} \\
1.29, \mathrm{Hb}\end{array}$ & $\begin{array}{c}6,8,7 \mathrm{~b} \\
6,7 \mathrm{a}\end{array}$ & 6,18 & 4 \\
\hline 8 & $44.7, \mathrm{CH}$ & 2.53 & $7 a, 19$ & 9 & 18 \\
\hline 9 & $213.4, \mathrm{C}$ & - & & & \\
\hline \multirow[t]{2}{*}{10} & $39.0, \mathrm{CH}_{2}$ & $2.53, \mathrm{Ha}$ & $10 \mathrm{~b}, 11 \mathrm{a}, 11 \mathrm{~b}$ & 9,11 & $11 b, 12$ \\
\hline & & $2.20, \mathrm{Hb}$ & $10 a, 11 a, 11 b$ & 9,11 & \\
\hline \multirow[t]{2}{*}{11} & 26.1, $\mathrm{CH}_{2}$ & $2.19, \mathrm{Ha}$ & $10 \mathrm{a}, 10 \mathrm{~b}, 12$ & $9,12,13$ & \\
\hline & & $2.15, \mathrm{Hb}$ & $10 \mathrm{a}, 10 \mathrm{~b}, 12$ & $9,10,12,13$ & $10 \mathrm{a}, 12,13$ \\
\hline 12 & $132.6, \mathrm{CH}$ & 5.51 ddd $(14.9,9.4,4.6)$ & $11 \mathrm{a}, 11 \mathrm{~b}, 13$ & $10,11,14$ & $10 \mathrm{a}, 11 \mathrm{~b}, 14$ \\
\hline 13 & $128.7, \mathrm{CH}$ & $5.30 \mathrm{dd}(14.9,9.1)$ & 12,14 & $11,12,14,15$ & $11 b, 20 b$ \\
\hline 14 & $50.2, \mathrm{CH}$ & 2.35 & $13,15,20 \mathrm{a}, 20 \mathrm{~b}$ & $12,13,15$ & 12,16 \\
\hline 15 & $69.9, \mathrm{CH}$ & 5.07 dq. $(9.1,6.1)$ & 14,16 & $1,13,14$ & $20 \mathrm{a}, 20 \mathrm{~b}$ \\
\hline 16 & $18.8, \mathrm{CH}_{3}$ & $1.30 \mathrm{~d}(6.1)$ & 15 & 14,15 & 14 \\
\hline 17 & $18.8, \mathrm{CH}_{3}$ & $1.17 \mathrm{~d}(6.7)$ & 4 & $3,4,5$ & $1^{\prime}, 2,3,5$ \\
\hline 18 & 17.1, $\mathrm{CH}_{3}$ & $0.97 \mathrm{~d}(6.7)$ & 6 & $5,6,7$ & 5,8 \\
\hline 19 & $18.5, \mathrm{CH}_{3}$ & $1.10 \mathrm{~d}(7.0)$ & 8 & $7,8,9$ & \\
\hline \multirow[t]{3}{*}{20} & $69.9, \mathrm{CH}_{2}$ & $3.93 \mathrm{dd}(9.8,4.7), \mathrm{Ha}$ & $14,20 \mathrm{~b}$ & $13,14,15,1^{\prime}$ & $15,1^{\prime \prime}$ \\
\hline & \multirow{2}{*}{\multicolumn{5}{|c|}{$\begin{array}{c}3.45 \mathrm{dd}(9.8,4.7), \mathrm{Hb} \quad 14,20 \mathrm{a} \\
\beta \text {-D-aldgarose }\end{array}$}} \\
\hline & & & & & \\
\hline $1^{\prime}$ & $101.2, \mathrm{CH}$ & $4.65 \mathrm{~d}(7.6)$ & $2^{\prime}$ & $5,5^{\prime}$ & $5,5^{\prime}, 17$ \\
\hline $2^{\prime}$ & 71.7, $\mathrm{CH}$ & 3.48 & $1^{\prime}$ & $1^{\prime}$ & $4^{\prime} \mathrm{b}, 8^{\prime}$ \\
\hline $3^{\prime}$ & $84.6, \mathrm{C}$ & - & & & \\
\hline \multirow[t]{2}{*}{$4^{\prime}$} & $41.2, \mathrm{CH}_{2}$ & 1.85 br. d (13.7), Нa & $4^{\prime} \mathrm{b}, 5^{\prime}$ & $2^{\prime}, 3^{\prime}, 7^{\prime}$ & $7^{\prime}$ \\
\hline & & $1.55, \mathrm{Hb}$ & $4^{\prime} \mathrm{a}, 5^{\prime}$ & $5^{\prime}, 6^{\prime}$ & $2^{\prime}, 7^{\prime}$ \\
\hline $5^{\prime}$ & $67.1, \mathrm{CH}$ & 3.90 & $6^{\prime}, 4^{\prime} \mathrm{a}, 4^{\prime} \mathrm{b}$ & $1^{\prime}$ & $1^{\prime}$ \\
\hline $6^{\prime}$ & $20.5, \mathrm{CH}_{3}$ & $1.23 \mathrm{~d}(6.4)$ & $5^{\prime}$ & $4^{\prime}, 5^{\prime}$ & \\
\hline $7^{\prime}$ & $81.3, \mathrm{CH}$ & $4.38 \mathrm{q}(6.4)$ & $8^{\prime}$ & $2^{\prime}, 3^{\prime}, 4^{\prime}$ & $4^{\prime} \mathrm{a}, 4^{\prime} \mathrm{b}$ \\
\hline $8^{\prime}$ & $13.6, \mathrm{CH}_{3}$ & $1.58 \mathrm{~d}(6.4)$ & $7^{\prime}$ & $3^{\prime}, 7^{\prime}$ & $2^{\prime}$ \\
\hline $9^{\prime}$ & $153.8, \mathrm{C}$ & - & & & \\
\hline \multicolumn{6}{|c|}{$\beta$-D-mycinose } \\
\hline $1 "$ & $101.0, \mathrm{CH}$ & $4.55 \mathrm{~d}(7.9)$ & $2^{\prime \prime}$ & $20,3^{\prime \prime}$ & 20a, 5", 7" \\
\hline $2^{\prime \prime}$ & $81.9, \mathrm{CH}$ & $3.04 \mathrm{dd}(7.9,2.6)$ & 1", 3" & 1", 7" & 4", 7" \\
\hline $3^{\prime \prime}$ & $79.9, \mathrm{CH}$ & $3.74 \mathrm{t}(2.6)$ & 2", 4" & 1", 2", 4", 5", 8" & \\
\hline $4 "$ & $72.7, \mathrm{CH}$ & $3.19 \mathrm{dd}(9.0,2.6)$ & 3", 5" & & 2", 6" \\
\hline $5^{\prime \prime}$ & $70.6, \mathrm{CH}$ & 3.51 & 4", 6" & $3^{\prime \prime}$ & $1 "$ \\
\hline $6^{\prime \prime}$ & $17.8, \mathrm{CH}_{3}$ & $1.25 \mathrm{~d}(6.1)$ & $5 "$ & 4", 5" & $4 "$ \\
\hline $7 "$ & $59.8, \mathrm{CH}_{3}$ & $3.53 \mathrm{~s}$ & & $2^{\prime \prime}$ & 1", 2" \\
\hline $8^{\prime \prime}$ & $61.7, \mathrm{CH}_{3}$ & $3.61 \mathrm{~s}$ & & $3 "$ & \\
\hline
\end{tabular}

* Indiscernible signals owing to overlapping or having complex multiplicity are reported without designating multiplicity. 


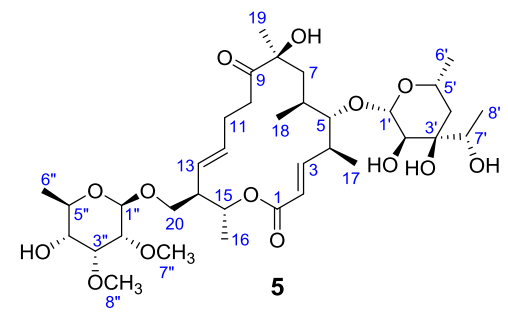

Table S5. NMR (400 MHz, $\left.\mathrm{CDCl}_{3}\right)$ assignments for 5.

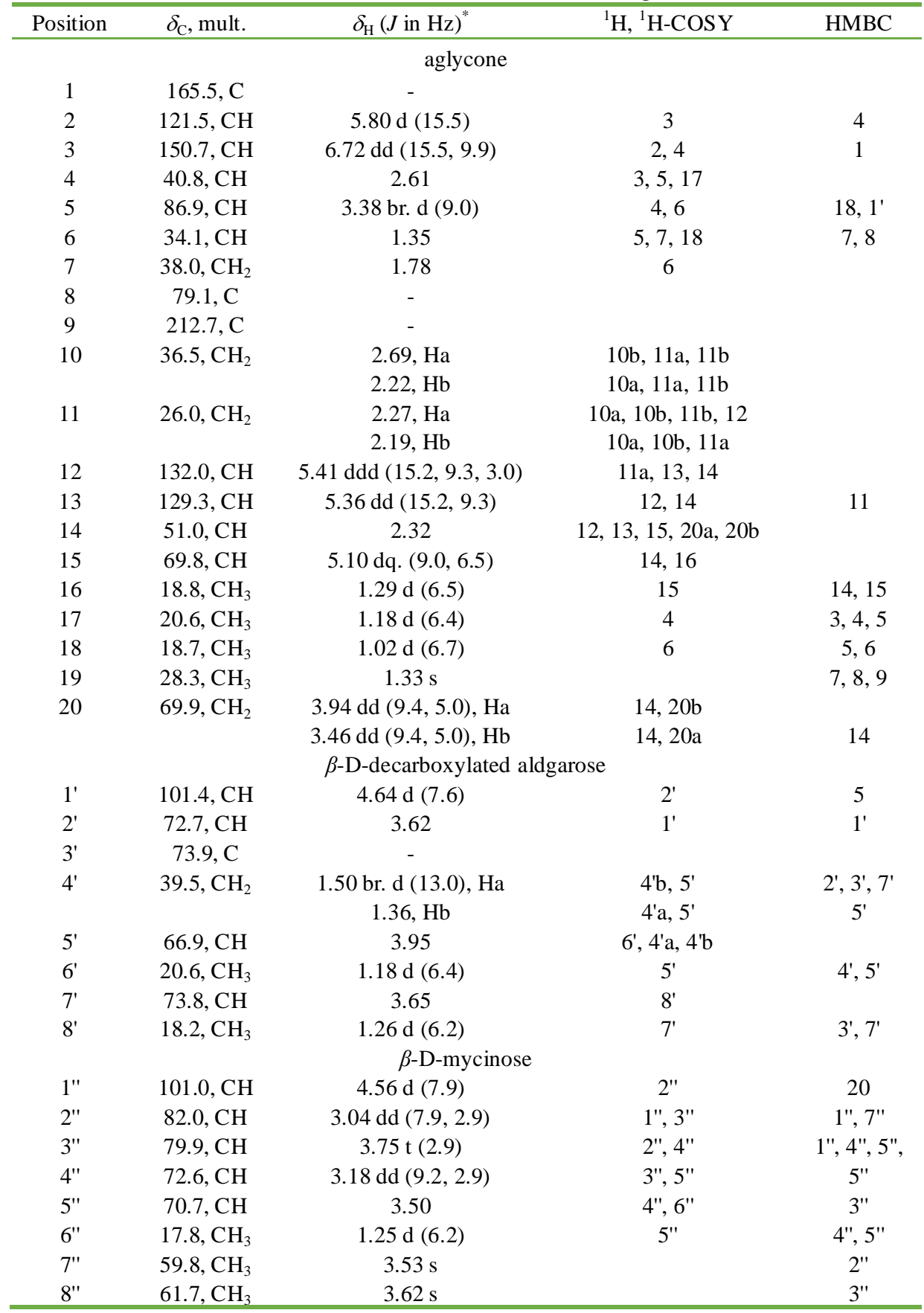

* Indiscernible signals owing to overlapping or having complex multiplicity are reported without designating multiplicity. 


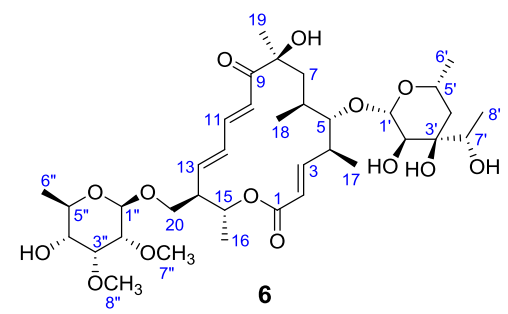

Table S6. NMR (400 MHz, $\left.\mathrm{CDCl}_{3}\right)$ assignments for 6.

\begin{tabular}{|c|c|c|c|c|}
\hline Position & $\delta_{\mathrm{C}}$, mult. & $\delta_{\mathrm{H}}(J \text { in } \mathrm{Hz})^{*}$ & ${ }^{1} \mathrm{H},{ }^{1} \mathrm{H}-\mathrm{COS} \mathrm{Y}$ & HMBC \\
\hline \multicolumn{5}{|c|}{ aglycone } \\
\hline 1 & $165.6, \mathrm{C}$ & - & & \\
\hline 2 & $121.5, \mathrm{CH}$ & $5.74 \mathrm{~d}(15.9)$ & 3 & 1,4 \\
\hline 3 & $151.6, \mathrm{CH}$ & $6.62 \mathrm{dd}(15.9,10.0)$ & 2,4 & 1 \\
\hline 4 & $40.9, \mathrm{CH}$ & 2.61 & $3,5,17$ & \\
\hline 5 & $87.5, \mathrm{CH}$ & 3.24 br. d (9.0) & 4,6 & $18,1^{\prime}$ \\
\hline 6 & $33.9, \mathrm{CH}$ & 1.34 & $5,7,18$ & 7,8 \\
\hline \multirow[t]{2}{*}{7} & $37.6, \mathrm{CH}_{2}$ & $1.87, \mathrm{Ha}$ & 6 & 8,9 \\
\hline & & $1.79, \mathrm{Hb}$ & & 6,18 \\
\hline 8 & 78.2, C & - & & \\
\hline 9 & $201.8, \mathrm{C}$ & - & & \\
\hline 10 & $122.0, \mathrm{CH}$ & $6.19 \mathrm{~d}(15.0)$ & 11 & 9,12 \\
\hline 11 & $144.1, \mathrm{CH}$ & $7.31 \mathrm{dd}(15.0,10.3)$ & 10,12 & 12,13 \\
\hline 12 & $133.0, \mathrm{CH}$ & $6.16 \mathrm{dd}(14.0,10.3)$ & 11,13 & $10,11,14$ \\
\hline 13 & $143.3, \mathrm{CH}$ & $6.15 \mathrm{dd}(14.0,9.0)$ & 12,14 & $11,12,14$ \\
\hline 14 & $51.3, \mathrm{CH}$ & 2.48 & $13,15,20 a, 20 b$ & \\
\hline 15 & $69.2, \mathrm{CH}$ & 5.06 dq. $(10.1,6.4)$ & 14,16 & 1 \\
\hline 16 & $18.6, \mathrm{CH}_{3}$ & $1.37 \mathrm{~d}(6.4)$ & 15 & 14,15 \\
\hline 17 & $20.7, \mathrm{CH}_{3}$ & $1.15 \mathrm{~d}(6.9)$ & 4 & $3,4,5$ \\
\hline 18 & $19.3, \mathrm{CH}_{3}$ & $1.00 \mathrm{~d}(6.9)$ & 6 & $5,6,7$ \\
\hline 19 & $28.0, \mathrm{CH}_{3}$ & $1.39 \mathrm{~s}$ & & $7,8,9$ \\
\hline \multirow[t]{3}{*}{20} & $68.4, \mathrm{CH}_{2}$ & $4.04 \mathrm{dd}(9.5,3.6), \mathrm{Ha}$ & $14,20 \mathrm{~b}$ & 13,15 \\
\hline & & $3.57 \mathrm{dd}(9.5,3.6), \mathrm{Hb}$ & $14,20 \mathrm{a}$ & $13,14,15$ \\
\hline & \multicolumn{4}{|c|}{$\beta$-D-decarboxylated aldgarose } \\
\hline $1^{\prime}$ & $101.1, \mathrm{CH}$ & $4.58 \mathrm{~d}(7.8)$ & $2^{\prime}$ & 5 \\
\hline $2^{\prime}$ & $72.7, \mathrm{CH}$ & 3.61 & $1^{\prime}$ & $1^{\prime}$ \\
\hline $3^{\prime}$ & 73.9, C & - & & \\
\hline \multirow[t]{2}{*}{$4^{\prime}$} & $39.6, \mathrm{CH}_{2}$ & $1.50 \mathrm{dd}(13.5,2.1), \mathrm{Ha}$ & $4^{\prime} b, 5^{\prime}$ & $2^{\prime}, 3^{\prime}, 7^{\prime}$ \\
\hline & & $1.34, \mathrm{Hb}$ & $4^{\prime} \mathrm{a}, 5^{\prime}$ & $5^{\prime}$ \\
\hline $5^{\prime}$ & $66.8, \mathrm{CH}$ & 3.93 & $6^{\prime}, 4^{\prime} \mathrm{a}, 4^{\prime} \mathrm{b}$ & \\
\hline $6^{\prime}$ & $20.7, \mathrm{CH}_{3}$ & $1.17 \mathrm{~d}(6.2)$ & $5^{\prime}$ & $4^{\prime}, 5^{\prime}$ \\
\hline $7^{\prime}$ & $73.9, \mathrm{CH}$ & 3.64 & $8^{\prime}$ & $3^{\prime}$ \\
\hline \multirow[t]{2}{*}{$8^{\prime}$} & $18.2, \mathrm{CH}_{3}$ & $1.28 \mathrm{~d}(6.5)$ & $7^{\prime}$ & $3^{\prime}, 7^{\prime}$ \\
\hline & & $\beta$-D-mycinose & & \\
\hline $1^{\prime \prime}$ & $101.1, \mathrm{CH}$ & $4.56 \mathrm{~d}(7.8)$ & $2^{\prime \prime}$ & 20 \\
\hline $2^{\prime \prime}$ & $82.0, \mathrm{CH}$ & $3.04 \mathrm{dd}(7.8,2.8)$ & 1", 3" & 1", 7" \\
\hline $3 "$ & $79.9, \mathrm{CH}$ & $3.76 \mathrm{t}(2.8)$ & 2", 4" & $1 ", 4 ", 5 ", 8^{\prime \prime}$ \\
\hline $4 "$ & $72.6, \mathrm{CH}$ & $3.18 \mathrm{dd}(9.2,2.8)$ & 3", 5" & \\
\hline $5^{\prime \prime}$ & $70.7, \mathrm{CH}$ & 3.51 & 4", 6" & $1 "$ \\
\hline $6^{\prime \prime}$ & $17.8, \mathrm{CH}_{3}$ & $1.27 \mathrm{~d}(6.6)$ & $5 "$ & $4 ", 5 "$ \\
\hline $7 "$ & $59.8, \mathrm{CH}_{3}$ & $3.52 \mathrm{~s}$ & & $2^{\prime \prime}$ \\
\hline $8^{\prime \prime}$ & $61.7, \mathrm{CH}_{3}$ & $3.62 \mathrm{~s}$ & & $3^{\prime \prime}$ \\
\hline
\end{tabular}

* Indiscernible signals owing to overlapping or having complex multiplicity are reported without designating multiplicity. 


\section{Acid hydrolysis of aldgamycin $\mathbf{J}$ (1)}

A solution of $1(7 \mathrm{mg})$ in $\mathrm{CH}_{3} \mathrm{OH}(1 \mathrm{ml})$ was added to $0.5 \mathrm{M} \mathrm{H}_{2} \mathrm{SO}_{4}(2 \mathrm{ml})$, and the solution was kept at $80{ }^{\circ} \mathrm{C}$ for 2 hours (Figure S1). Then the solution was neutralized with $\mathrm{Ba}(\mathrm{OH})_{2}$ saturated with $\mathrm{H}_{2} \mathrm{O}$ and the precipitate was filtered off. The filtrate was partitioned with EtOAc $(3 \times 3 \mathrm{ml})$. The aqueous layer was concentrated and then dissolved in $\mathrm{H}_{2} \mathrm{O}$. The solution was analyzed using HPLC (Shimadzu Co. Ltd., Kyoto, Japan; Phenomenex 5 $\mu$-NH $4.6 \times 250 \mathrm{~mm}, \mathrm{CH}_{3} \mathrm{CN}: \mathrm{H}_{2} \mathrm{O}=85: 15,1 \mathrm{ml} / \mathrm{min}$ ) with a ELSD detector (Grace Co. Ltd., Columbia, USA; tube temp: $80^{\circ} \mathrm{C}$, Gas: $1.2 \mathrm{ml} / \mathrm{min}$ ) (Figure S2). Then the solution was prepared using HPLC (Shimadzu Co. Ltd., Kyoto, Japan; Phenomenex $5 \mu-\mathrm{NH}_{2}, 4.6 \times 250 \mathrm{~mm}, \mathrm{CH}_{3} \mathrm{CN}: \mathrm{H}_{2} \mathrm{O}=85: 15,1 \mathrm{ml} / \mathrm{min}$ ) with RI detector (Shimadzu Co. Ltd., Kyoto, Japan) to yield $\mathbf{1 a}$ and $\mathbf{1 b}$. Compounds $\mathbf{1 a}$ and $\mathbf{1 b}$ were identified as decarboxylated aldgarose and mycinose respectively on the basis of HR-MS and ${ }^{1} \mathrm{H}$ NMR data. The optical rotations of $\mathbf{1 a}$ and $\mathbf{1 b}$ were $[\alpha]_{\mathrm{D}}^{30}-20.3(c 0.3$, $\left.\mathrm{H}_{2} \mathrm{O}\right)$ and $[\alpha]_{\mathrm{D}}^{30}-26.0\left(c 0.2, \mathrm{H}_{2} \mathrm{O}\right)$, respectively.

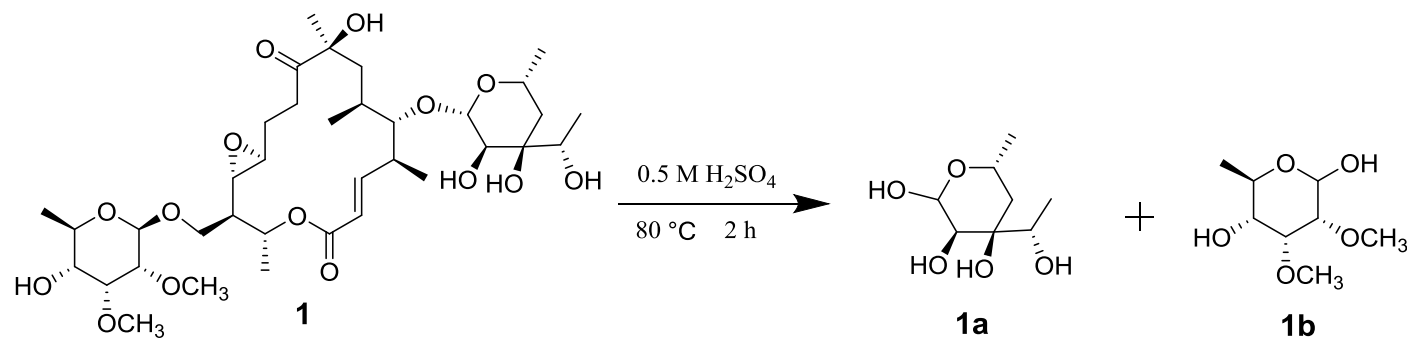

Figure S1. The process of acid hydrolysis of aldgamycin J (1)

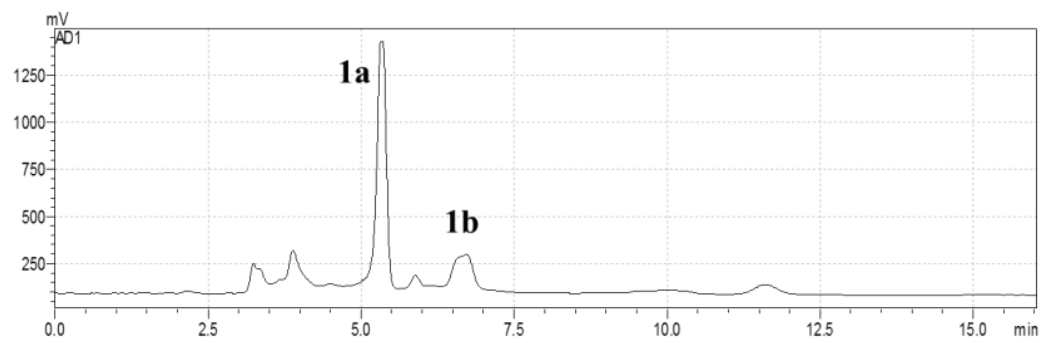

Figure S2. The aqueous layer of acid hydrolysis product of $\mathbf{1}$ was analyzed by HPLC with ELSD detector 


\section{The 1D and 2D NMR spectra of 1-8}

The 1D and 2D NMR spectra of aldgamycin J (1)

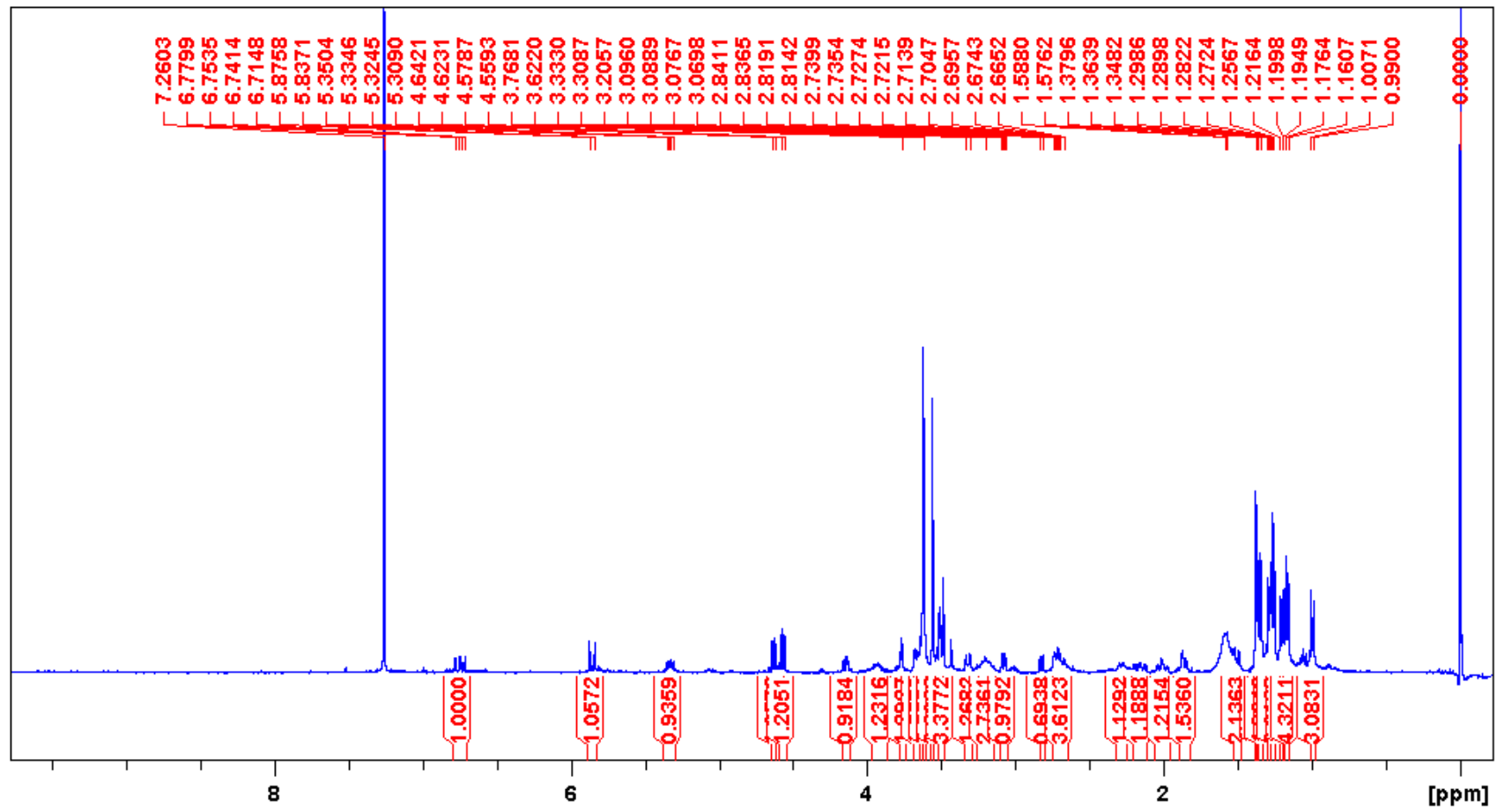

Figure S3. ${ }^{1} \mathrm{H}$ NMR $\left(400 \mathrm{MHz}, \mathrm{CDCl}_{3}\right)$ spectrum for aldgamycin J (1)

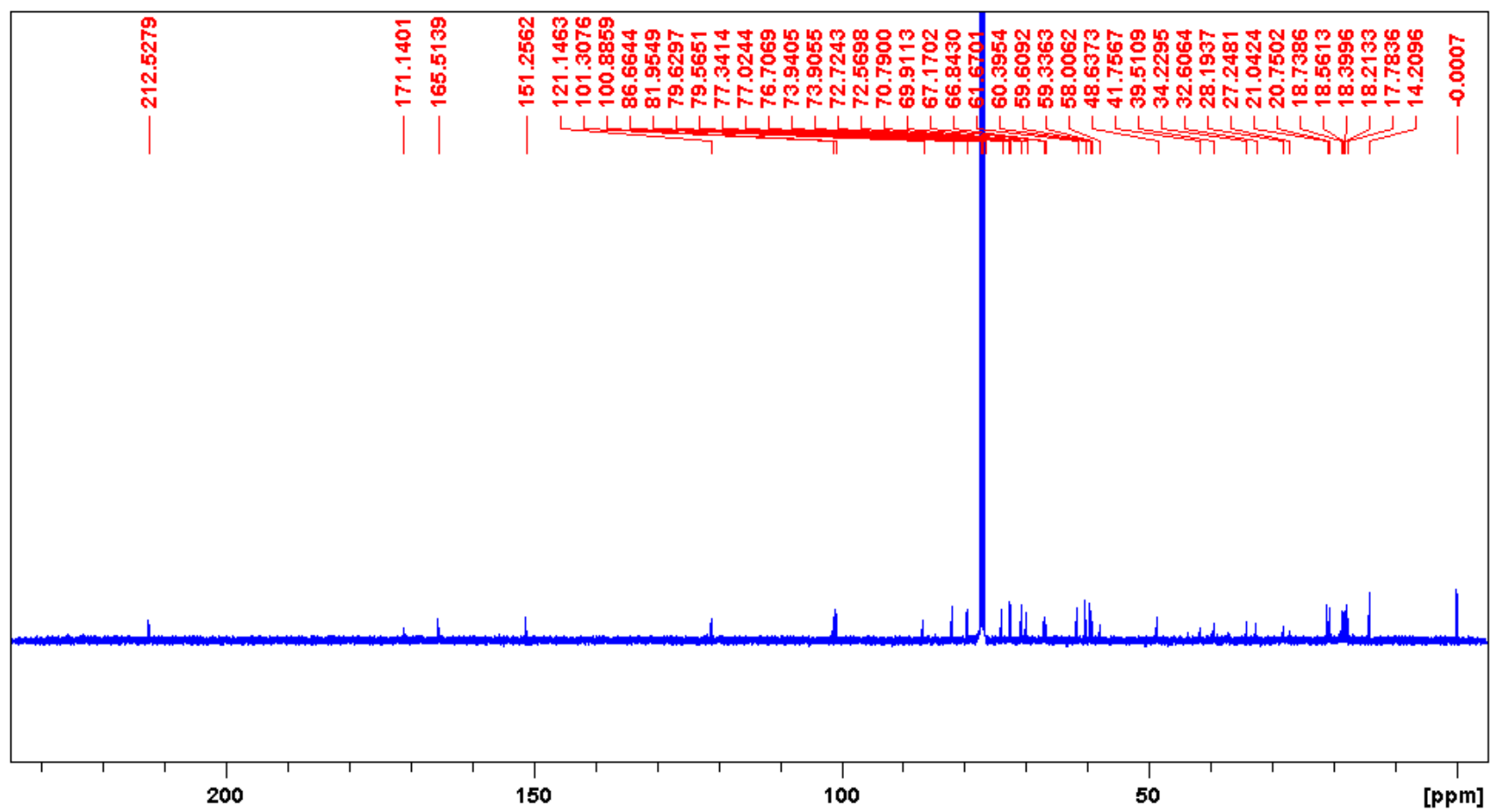

Figure S4. ${ }^{13} \mathrm{C}$ NMR $\left(100 \mathrm{MHz}, \mathrm{CDCl}_{3}\right)$ spectrum for aldgamycin J (1) 


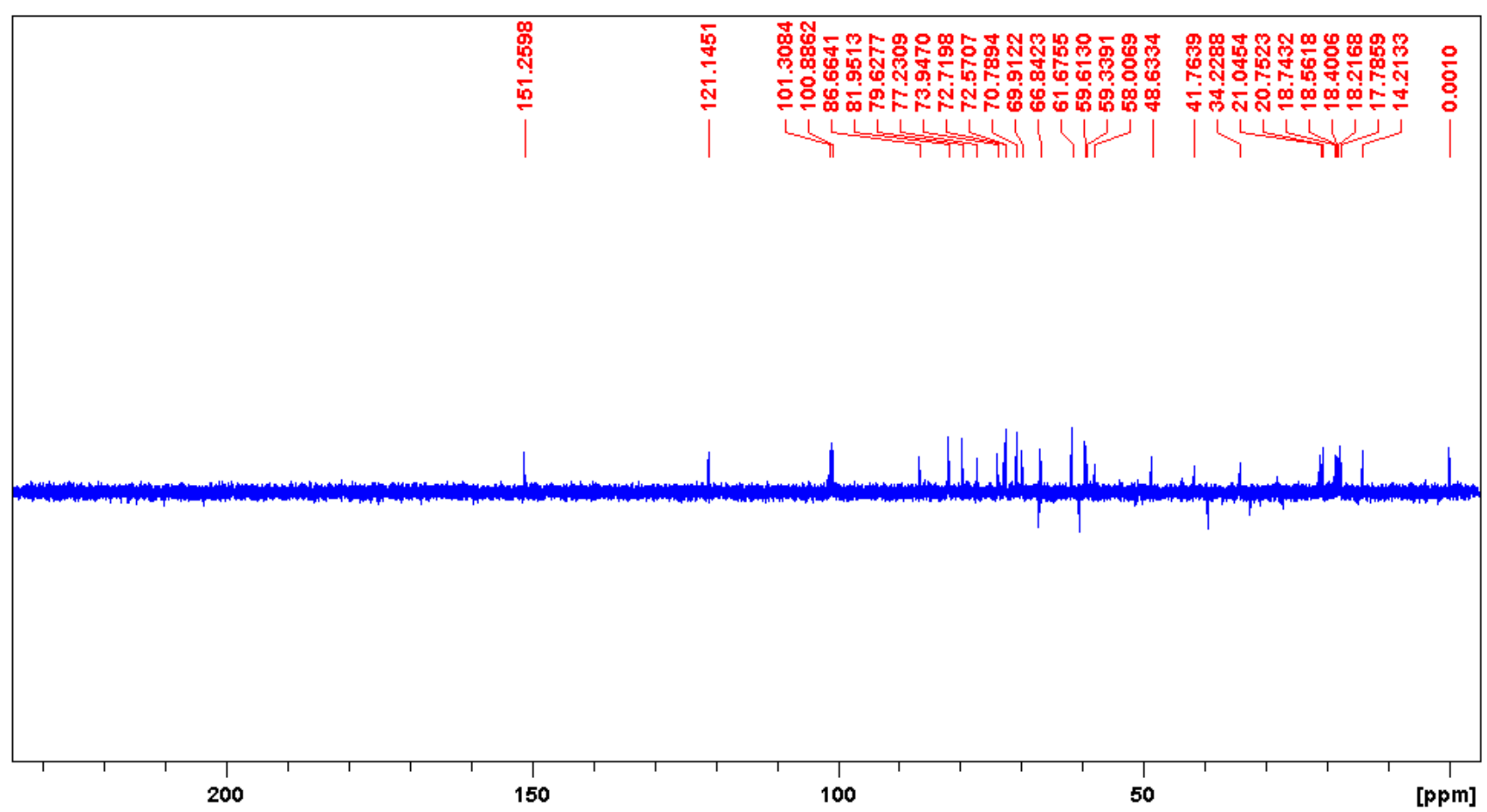

Figure S5. DEPT135 (100 MHz, $\left.\mathrm{CDCl}_{3}\right)$ spectrum for aldgamycin J (1)

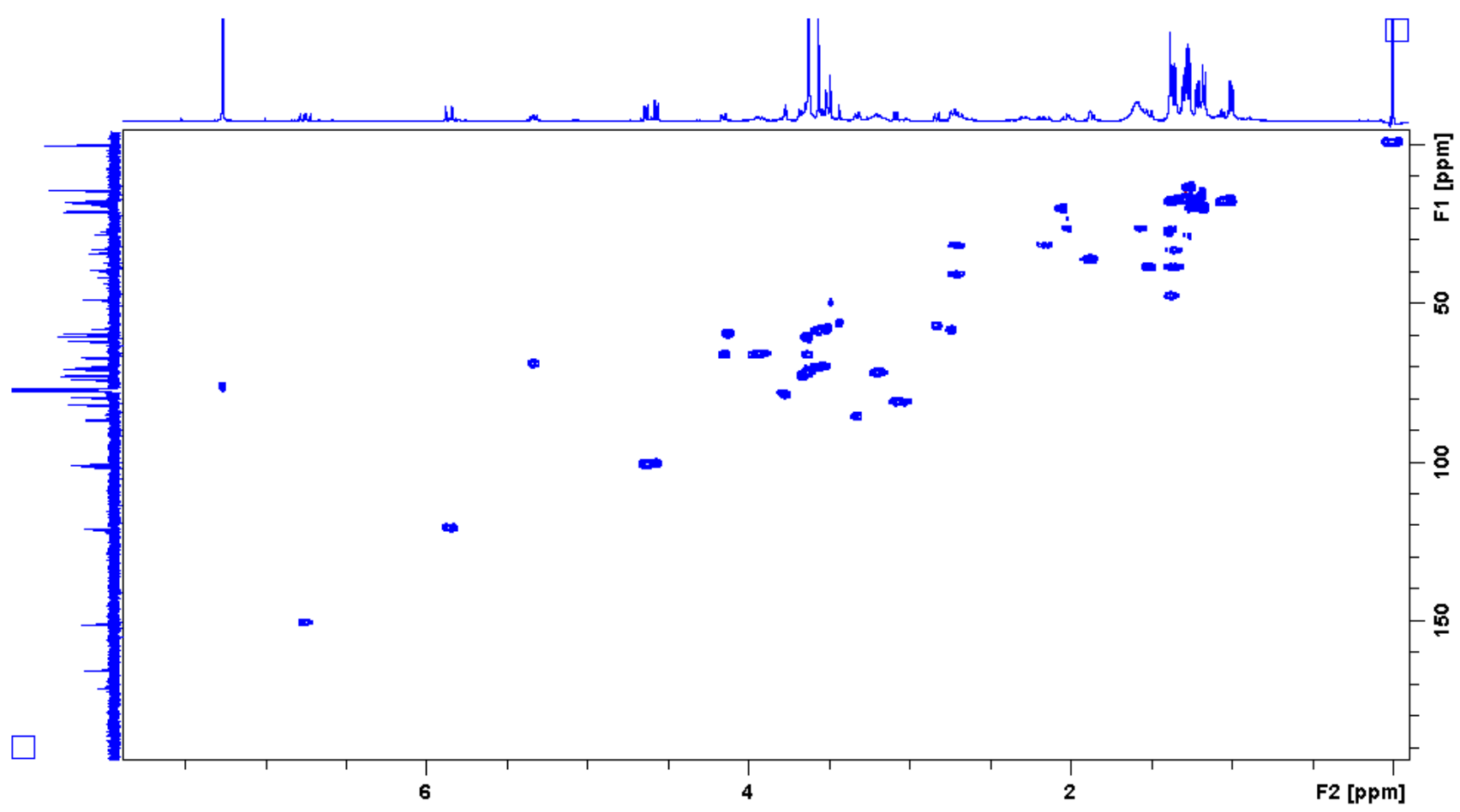

Figure S6. HSQC $\left(\mathrm{CDCl}_{3}\right)$ spectrum for aldgamycin J (1) 


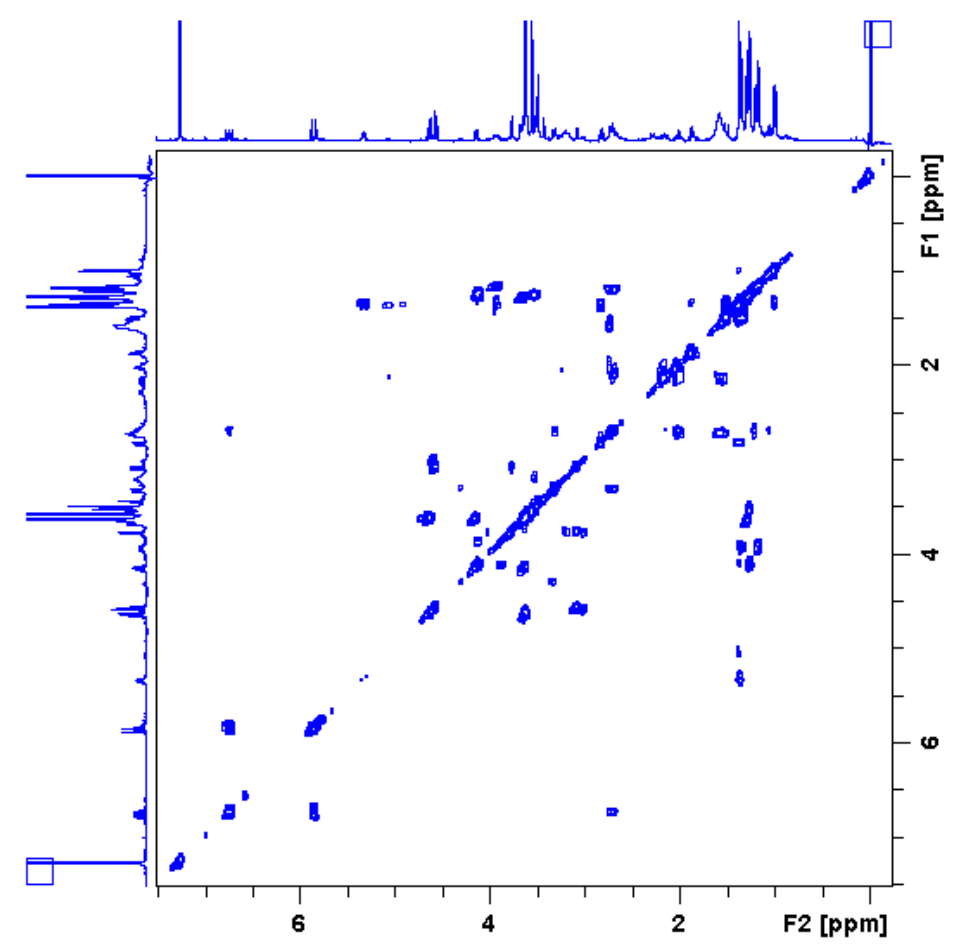

Figure S7. ${ }^{1} \mathrm{H}-{ }^{1} \mathrm{H} \operatorname{COSY}\left(\mathrm{CDCl}_{3}\right)$ spectrum for aldgamycin J (1)

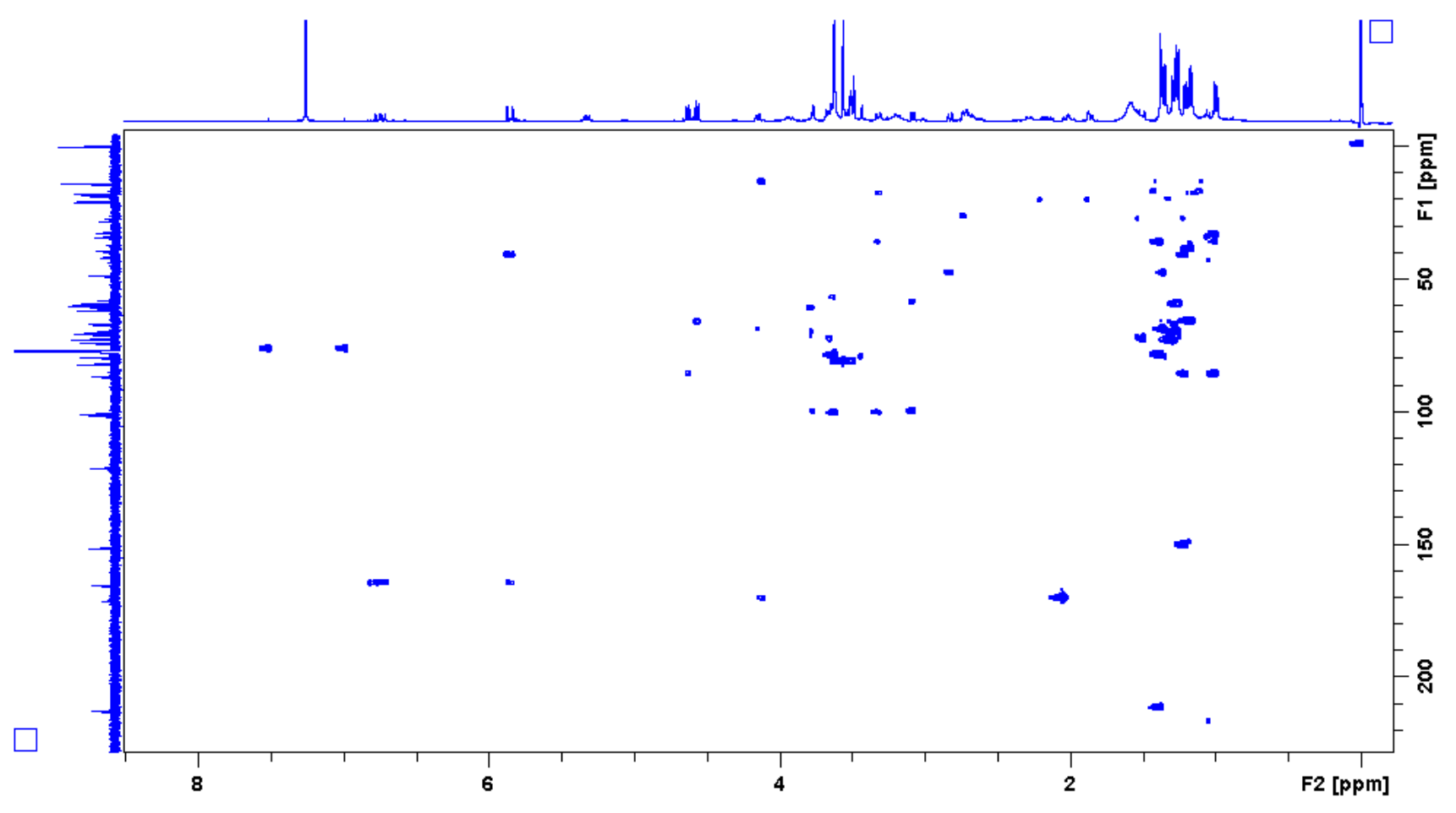

Figure S8. HMBC $\left(\mathrm{CDCl}_{3}\right)$ spectrum for aldgamycin J (1) 


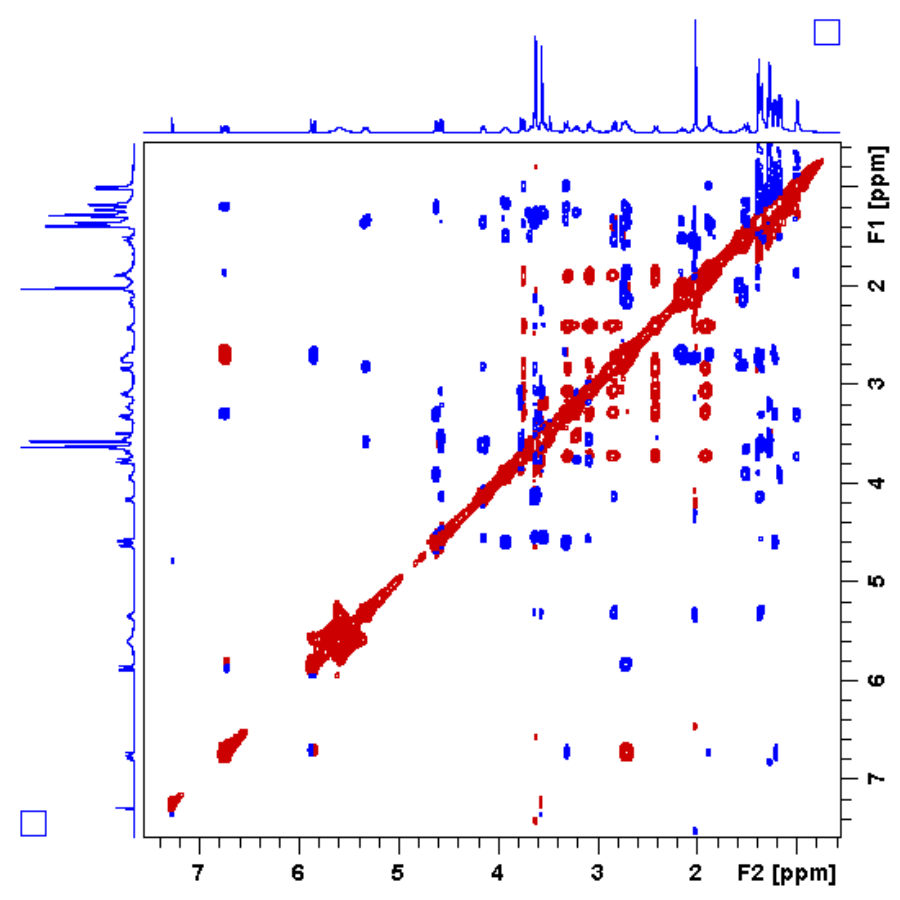

Figure S9. ROESY $\left(\mathrm{CDCl}_{3}\right)$ spectrum for aldgamycin J (1)

The 1D and 2D NMR spectra of aldgamycin K (2)

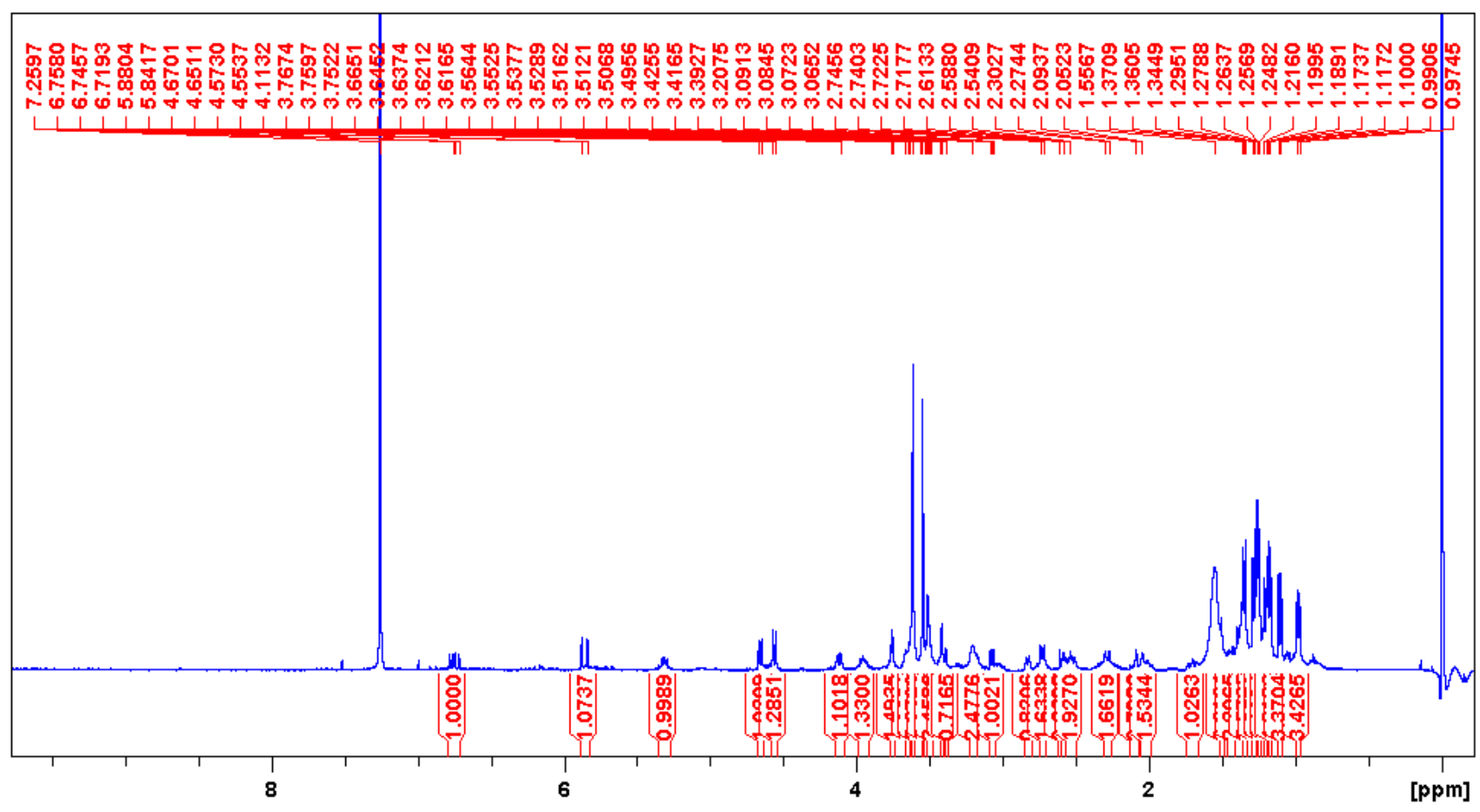

Figure S10. ${ }^{1} \mathrm{H}$ NMR $\left(400 \mathrm{MHz}, \mathrm{CDCl}_{3}\right)$ spectrum for aldgamycin $\mathrm{K}$ (2) 


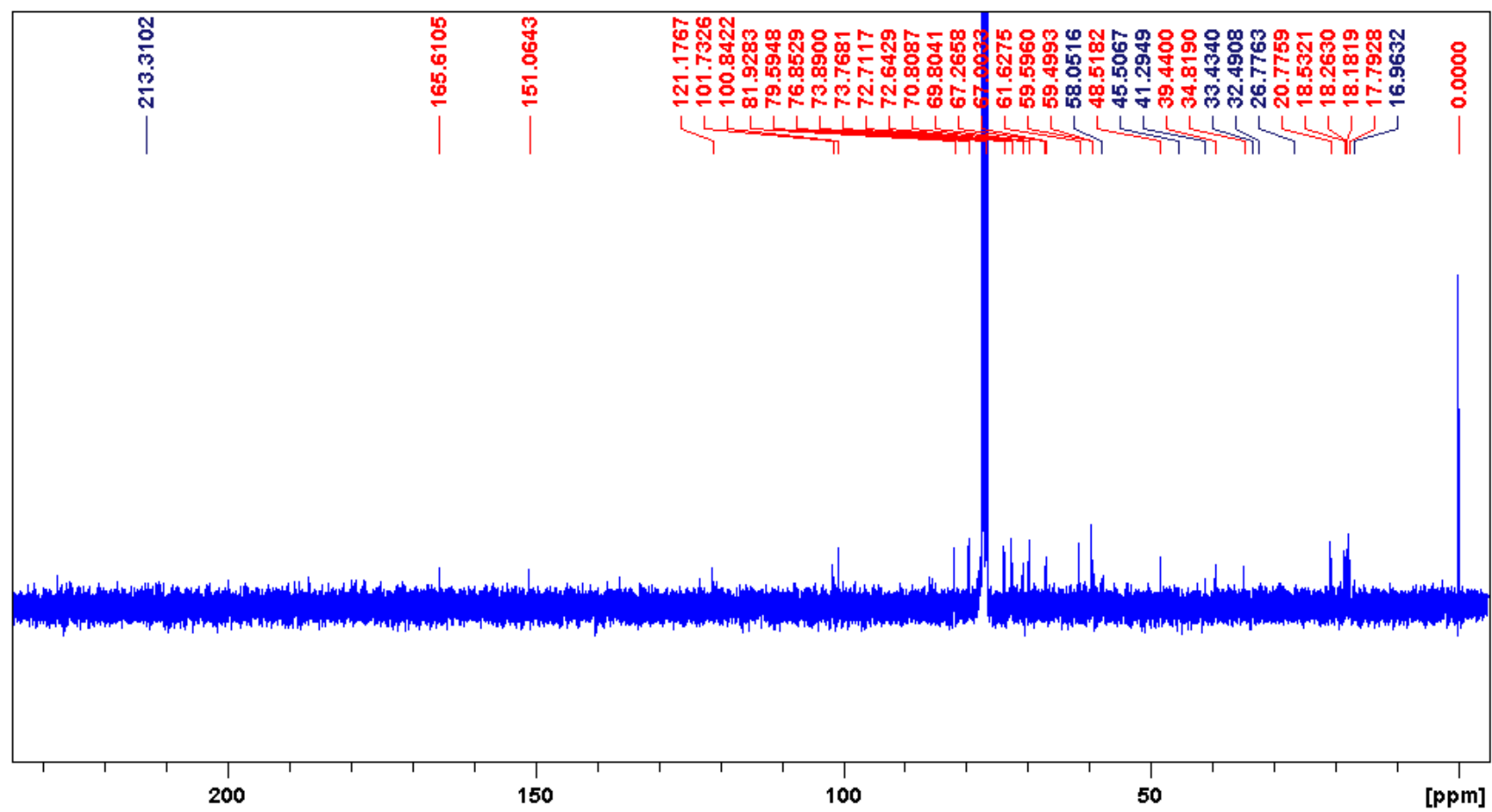

Figure S11. ${ }^{13} \mathrm{C}$ NMR $\left(100 \mathrm{MHz}, \mathrm{CDCl}_{3}\right)$ spectrum for aldgamycin $\mathrm{K}$ (2)

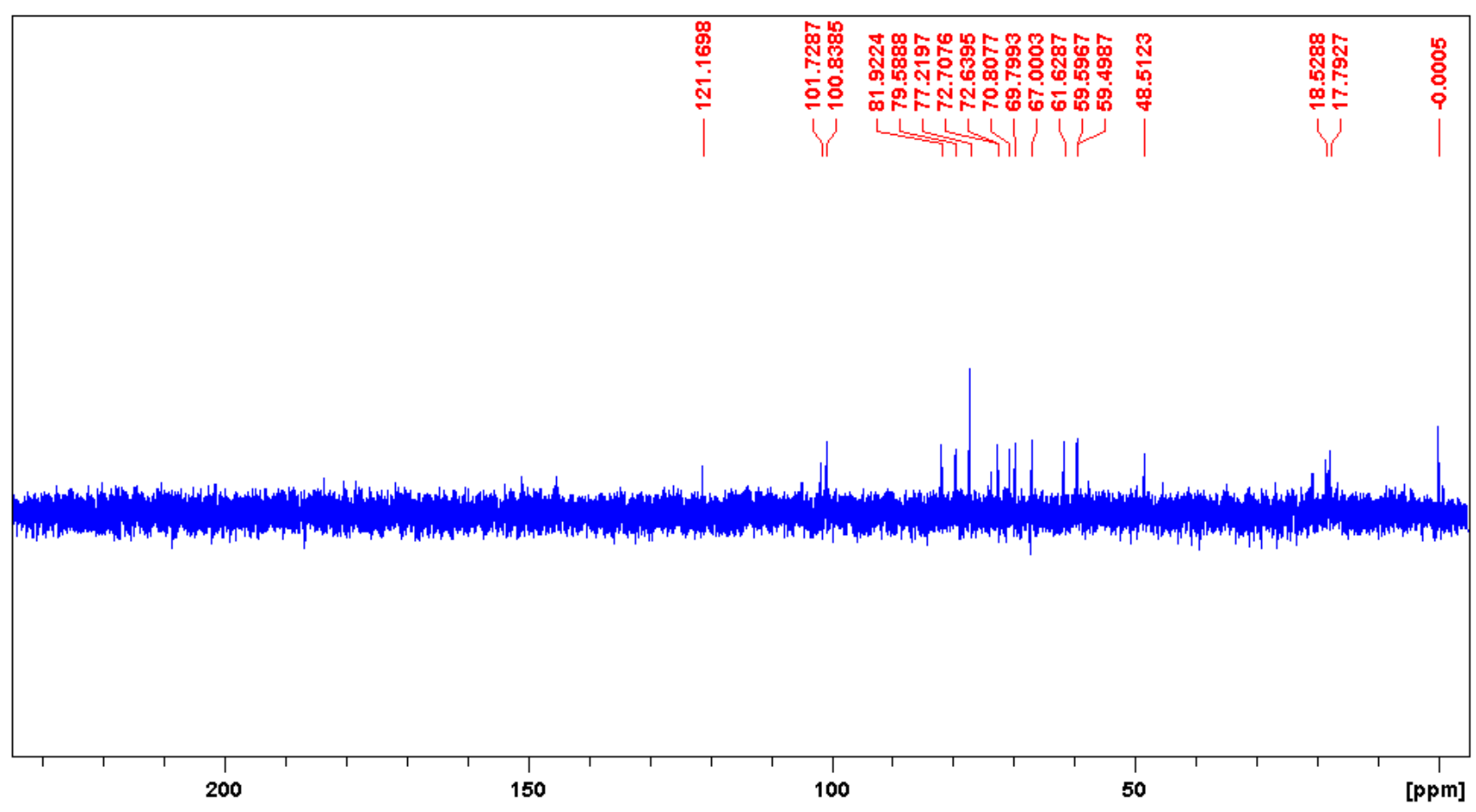

Figure S12. DEPT135 (100 MHz, $\left.\mathrm{CDCl}_{3}\right)$ spectrum for aldgamycin $\mathrm{K}(\mathbf{2})$ 


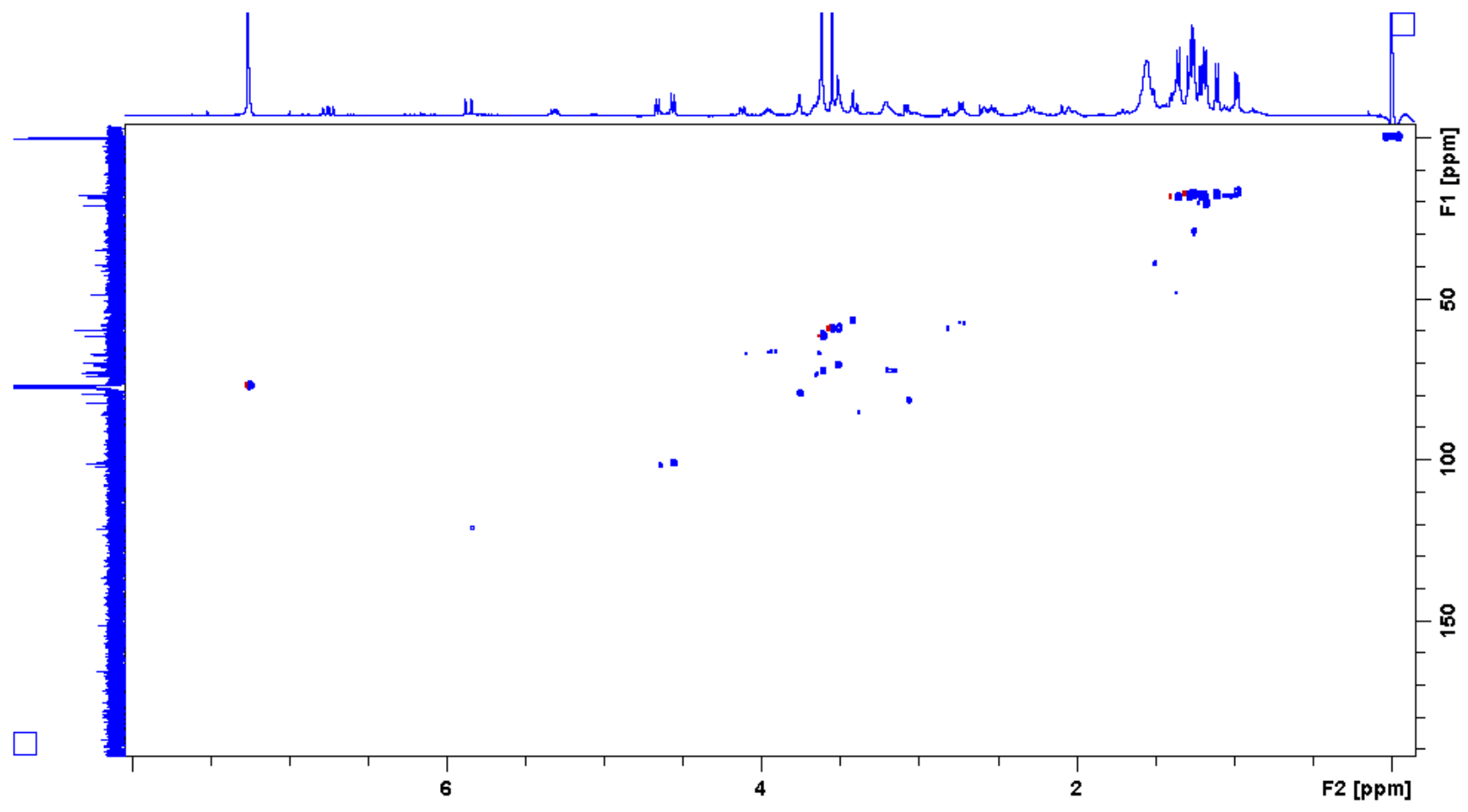

Figure S13. HSQC $\left(\mathrm{CDCl}_{3}\right)$ spectrum for aldgamycin $\mathrm{K}(\mathbf{2})$

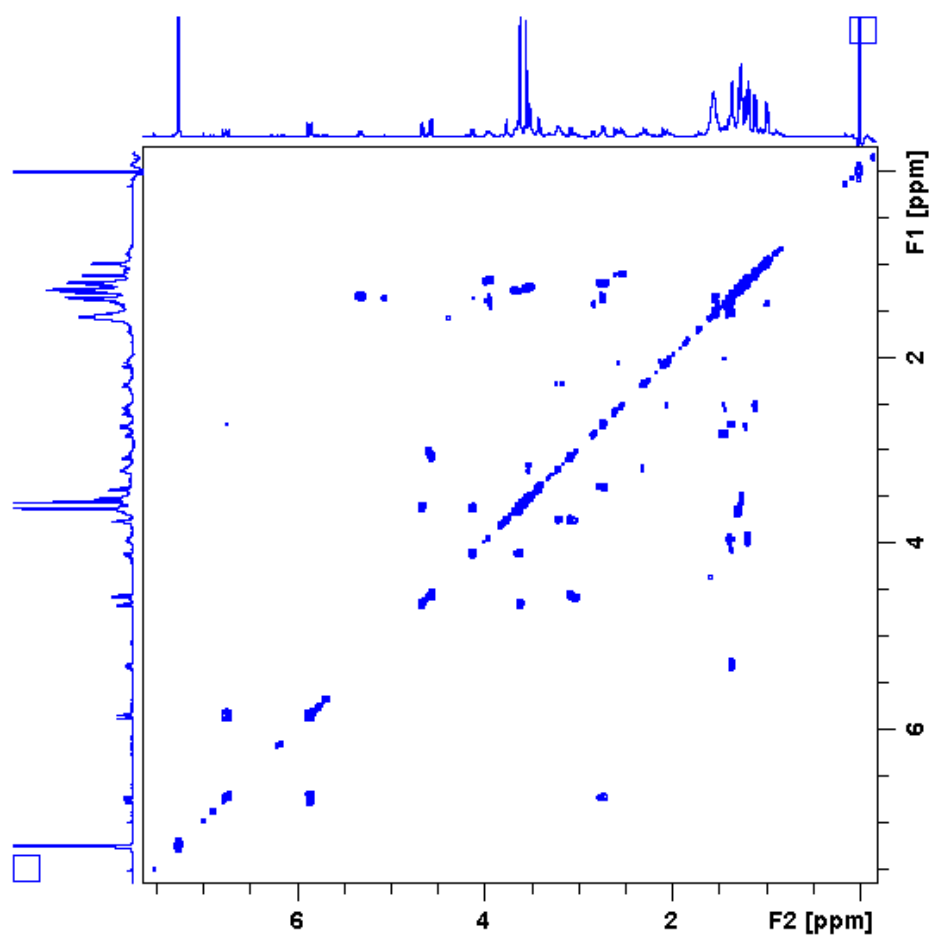

Figure S14. ${ }^{1} \mathrm{H}-{ }^{1} \mathrm{H} \operatorname{COSY}\left(\mathrm{CDCl}_{3}\right)$ spectrum for aldgamycin $\mathrm{K}(\mathbf{2})$ 


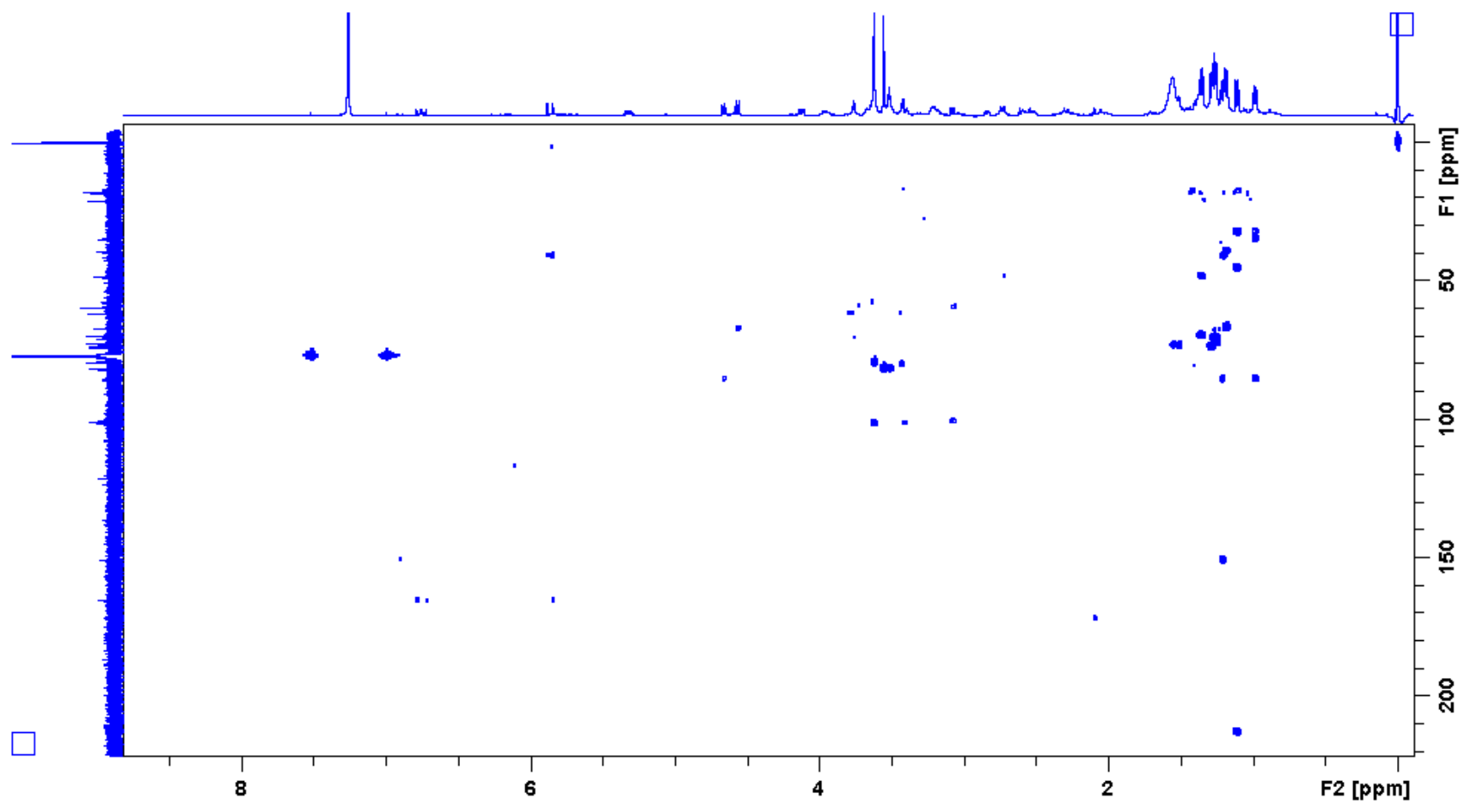

Figure S15. $\mathrm{HMBC}\left(\mathrm{CDCl}_{3}\right)$ spectrum for aldgamycin $\mathrm{K}(\mathbf{2})$

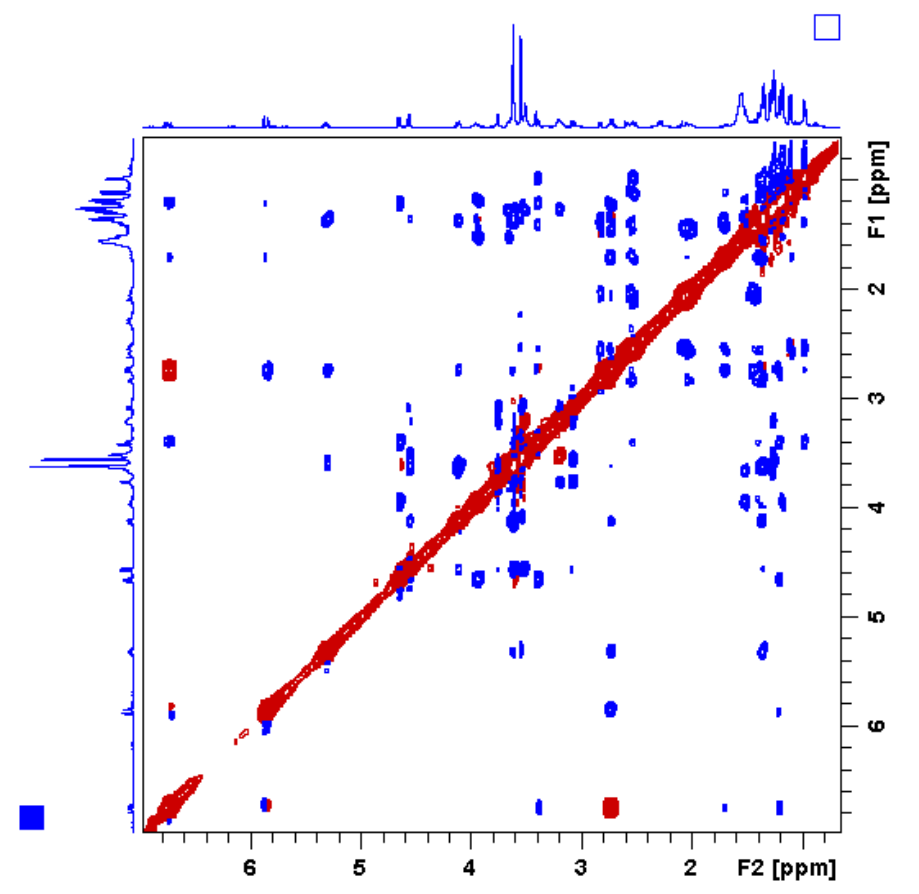

Figure S16. ROESY $\left(\mathrm{CDCl}_{3}\right)$ spectrum for aldgamycin $\mathrm{K}(\mathbf{2})$ 
The 1D and 2D NMR spectra of aldgamycin L (3)

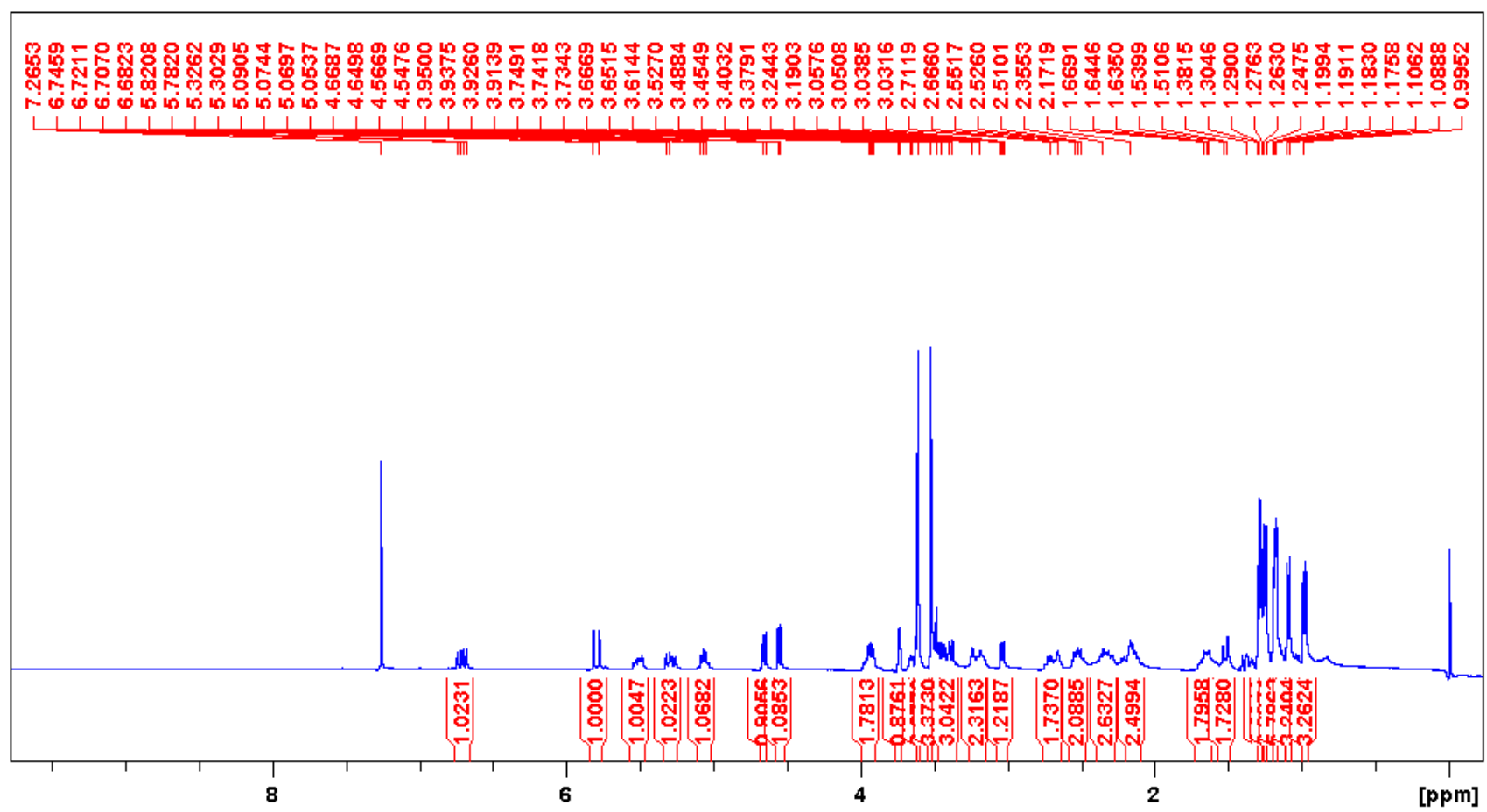

Figure S17. ${ }^{1} \mathrm{H}$ NMR $\left(400 \mathrm{MHz}, \mathrm{CDCl}_{3}\right)$ spectrum for aldgamycin L (3)

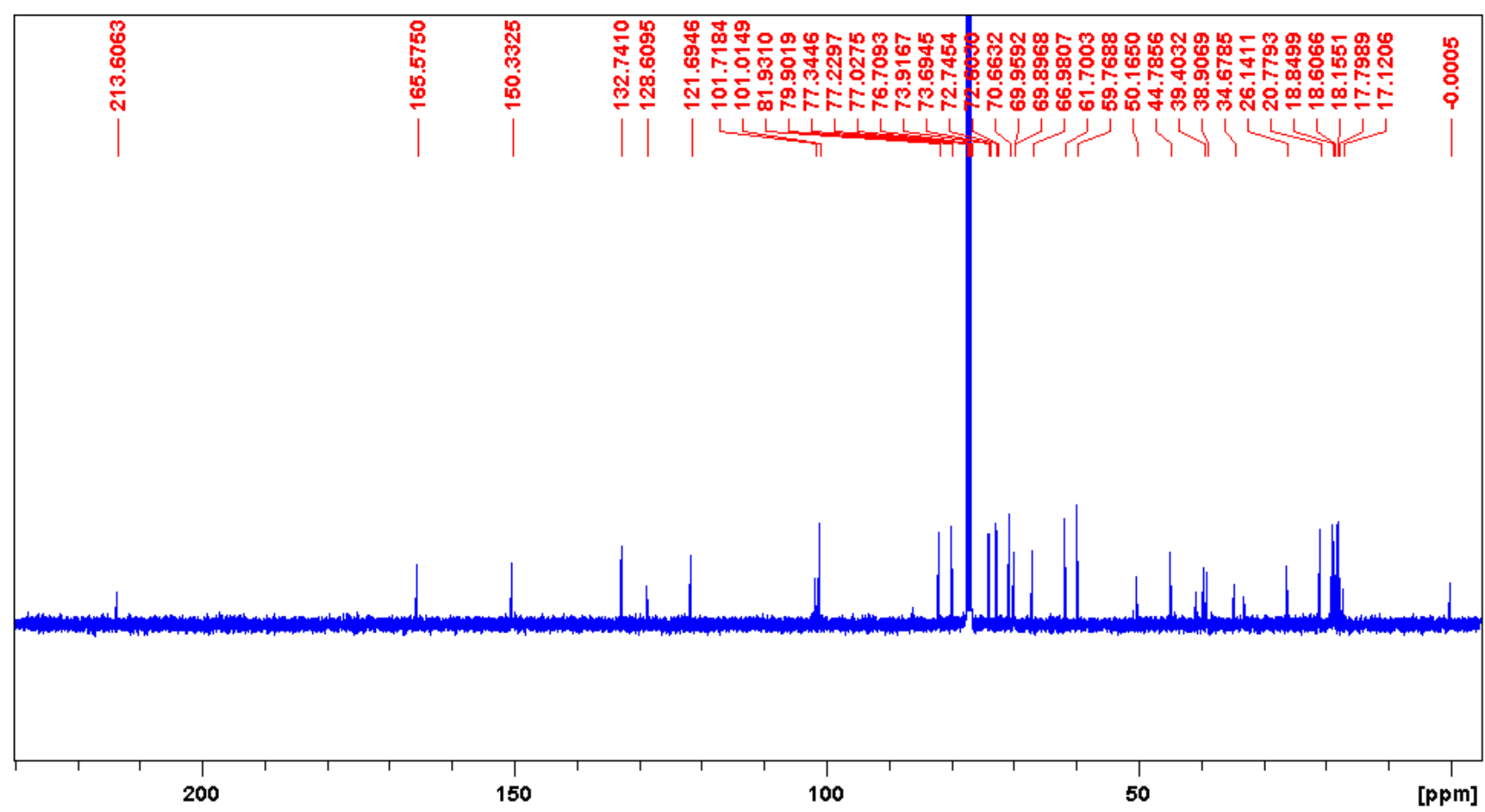

Figure S18. ${ }^{13} \mathrm{C}$ NMR (100 MHz, $\left.\mathrm{CDCl}_{3}\right)$ spectrum for aldgamycin $\mathrm{L}(\mathbf{3})$ 


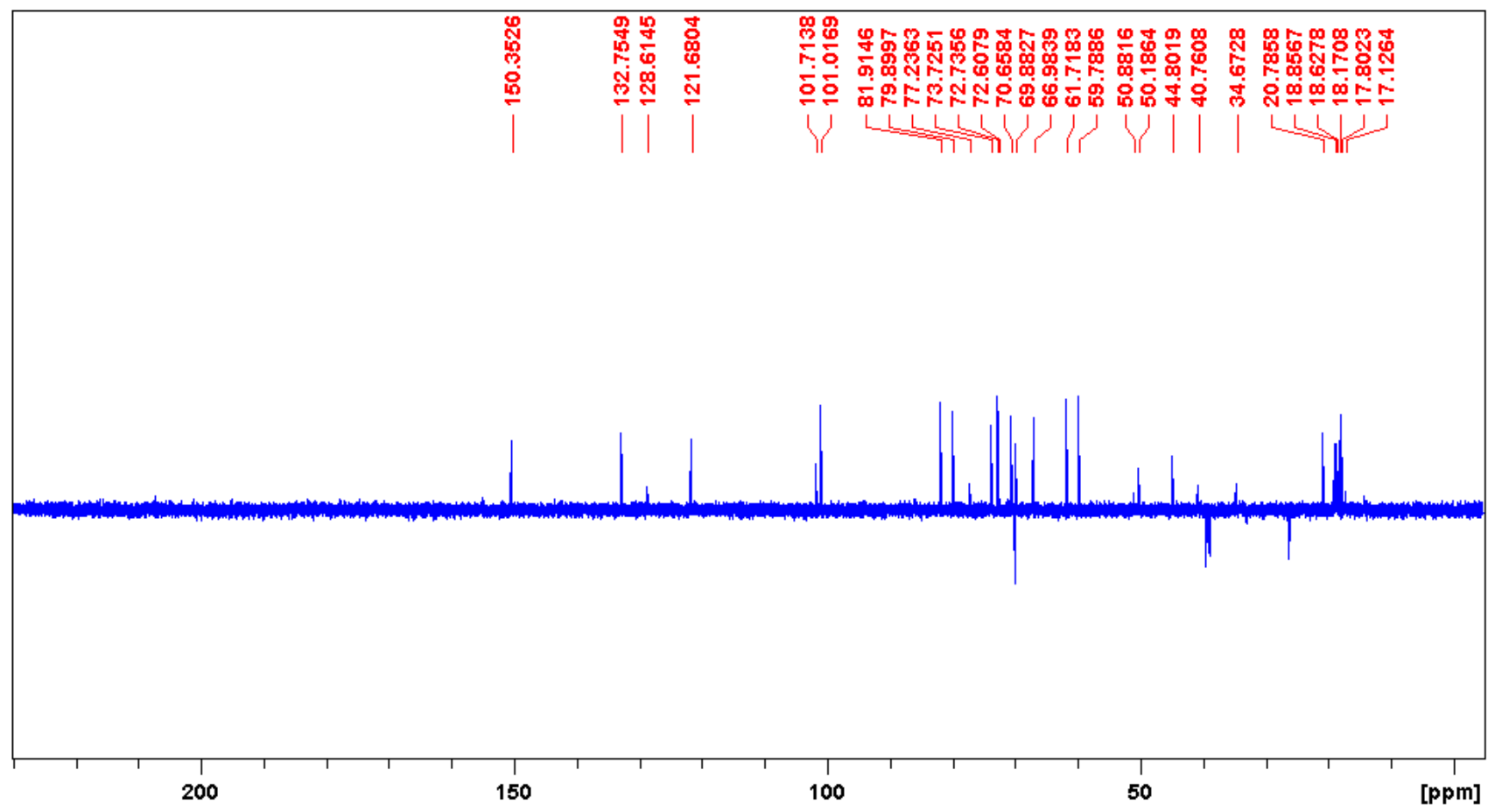

Figure S19 DEPT135 (100 MHz, $\left.\mathrm{CDCl}_{3}\right)$ spectrum for aldgamycin L (3)

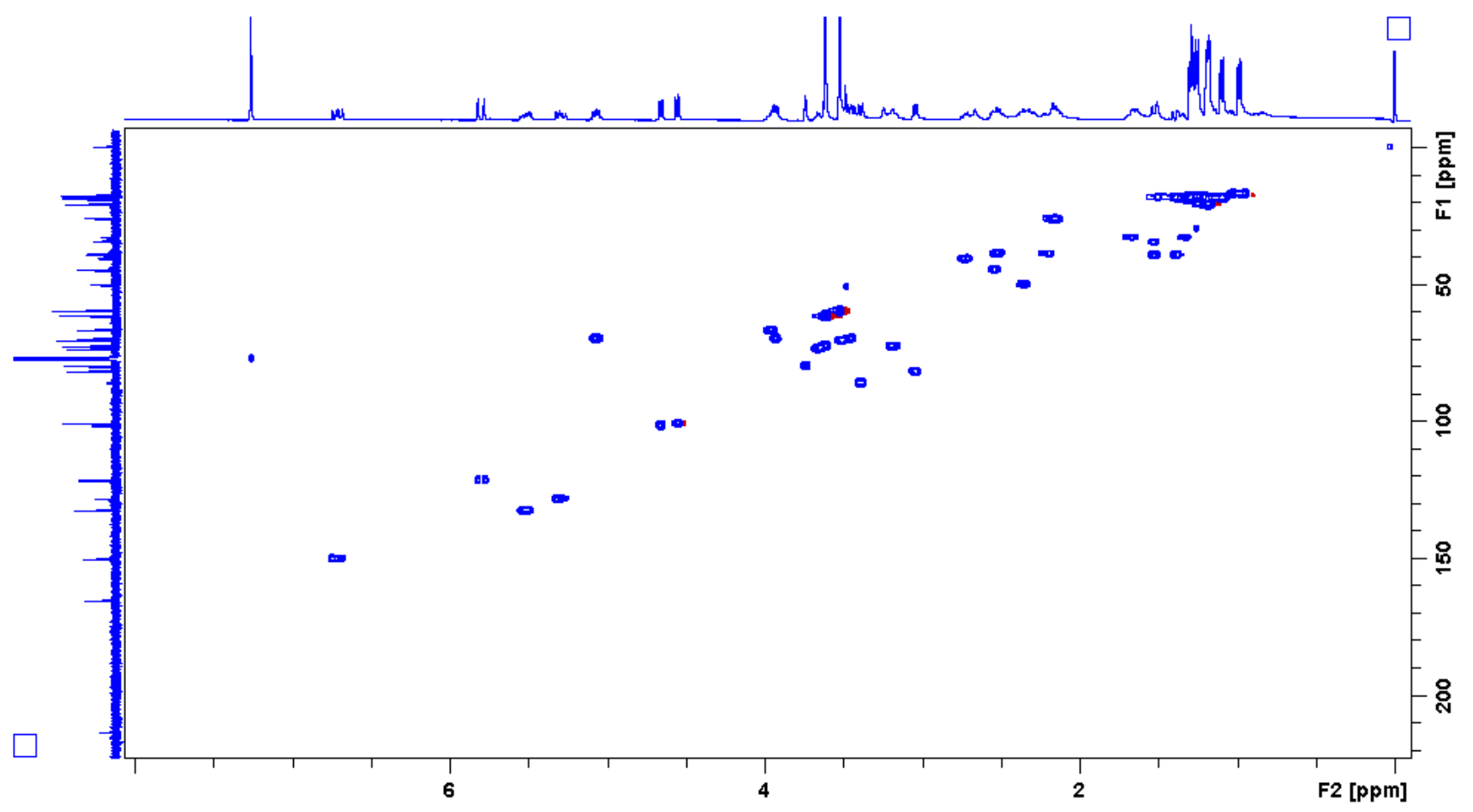

Figure S20. HSQC $\left(\mathrm{CDCl}_{3}\right)$ spectrum for aldgamycin $\mathrm{L}(\mathbf{3})$ 


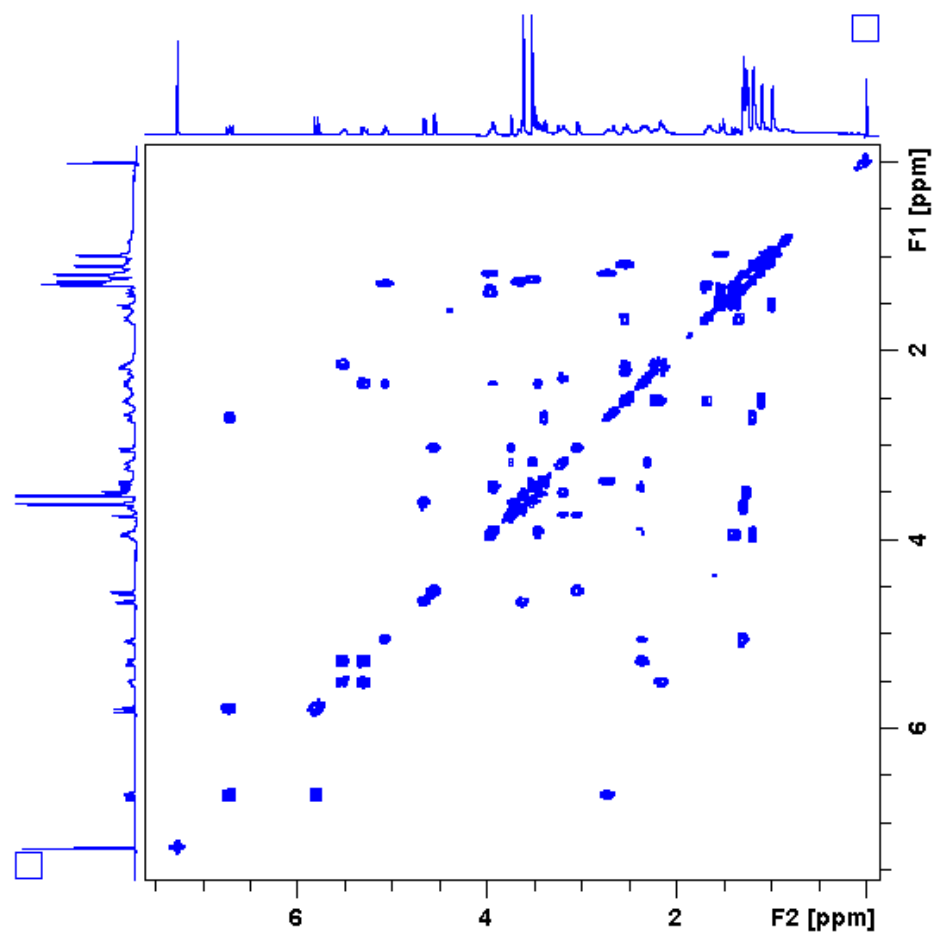

Figure S21. ${ }^{1} \mathrm{H}-{ }^{1} \mathrm{H} \operatorname{COSY}\left(\mathrm{CDCl}_{3}\right)$ spectrum for aldgamycin $\mathrm{L}(\mathbf{3})$

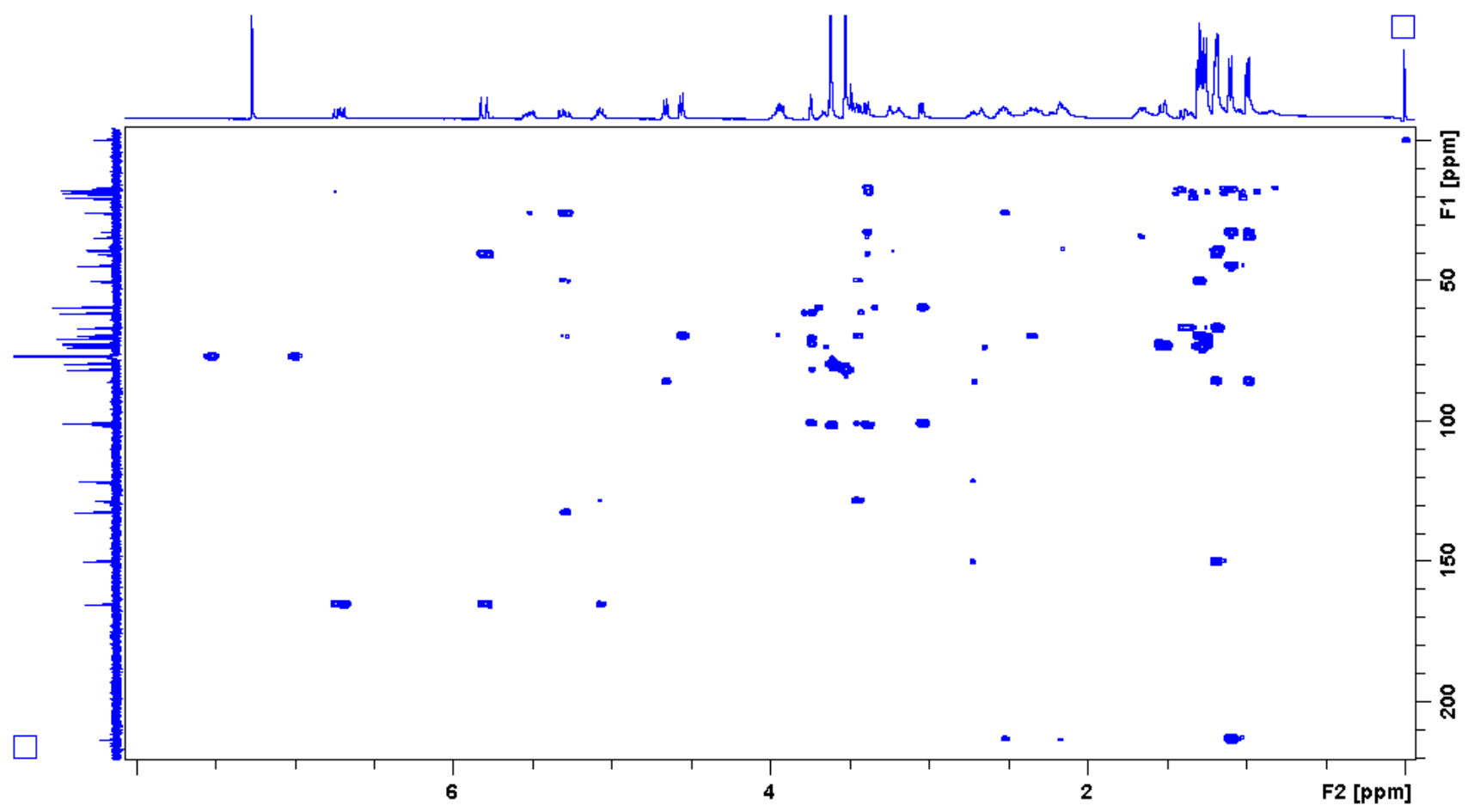

Figure S22. $\mathrm{HMBC}\left(\mathrm{CDCl}_{3}\right)$ spectrum for aldgamycin L (3) 


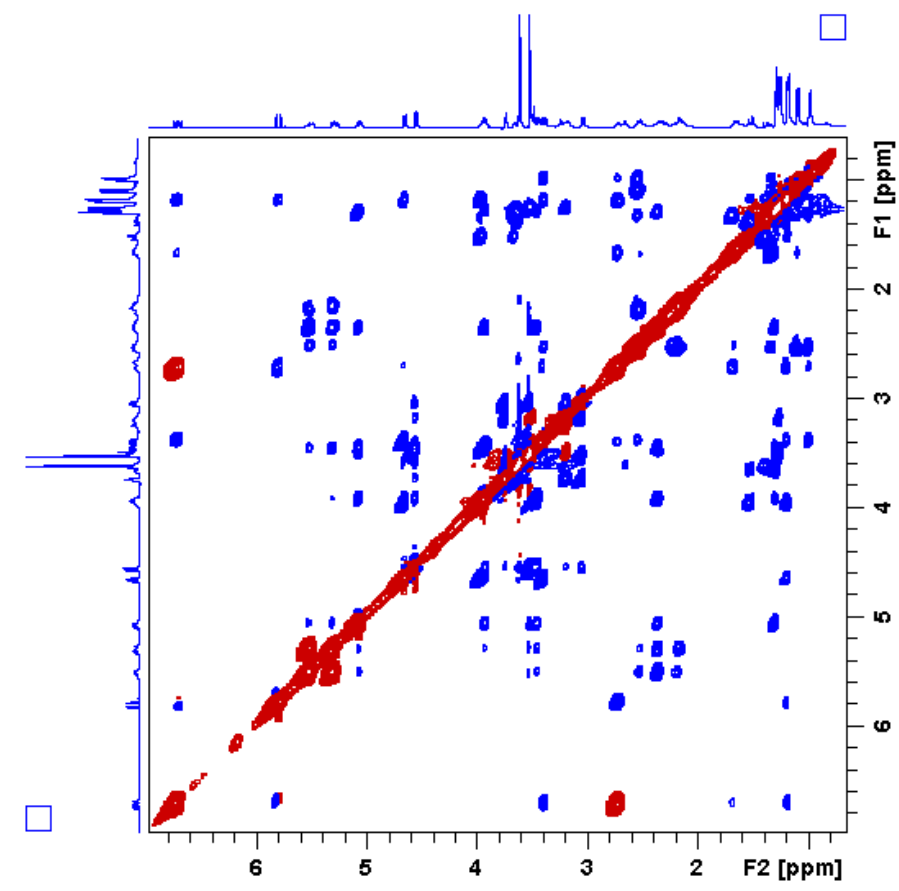

Figure S23. ROESY $\left(\mathrm{CDCl}_{3}\right)$ spectrum for aldgamycin $\mathrm{L}(\mathbf{3})$

The 1D and 2D NMR spectra of aldgamycin M (4)

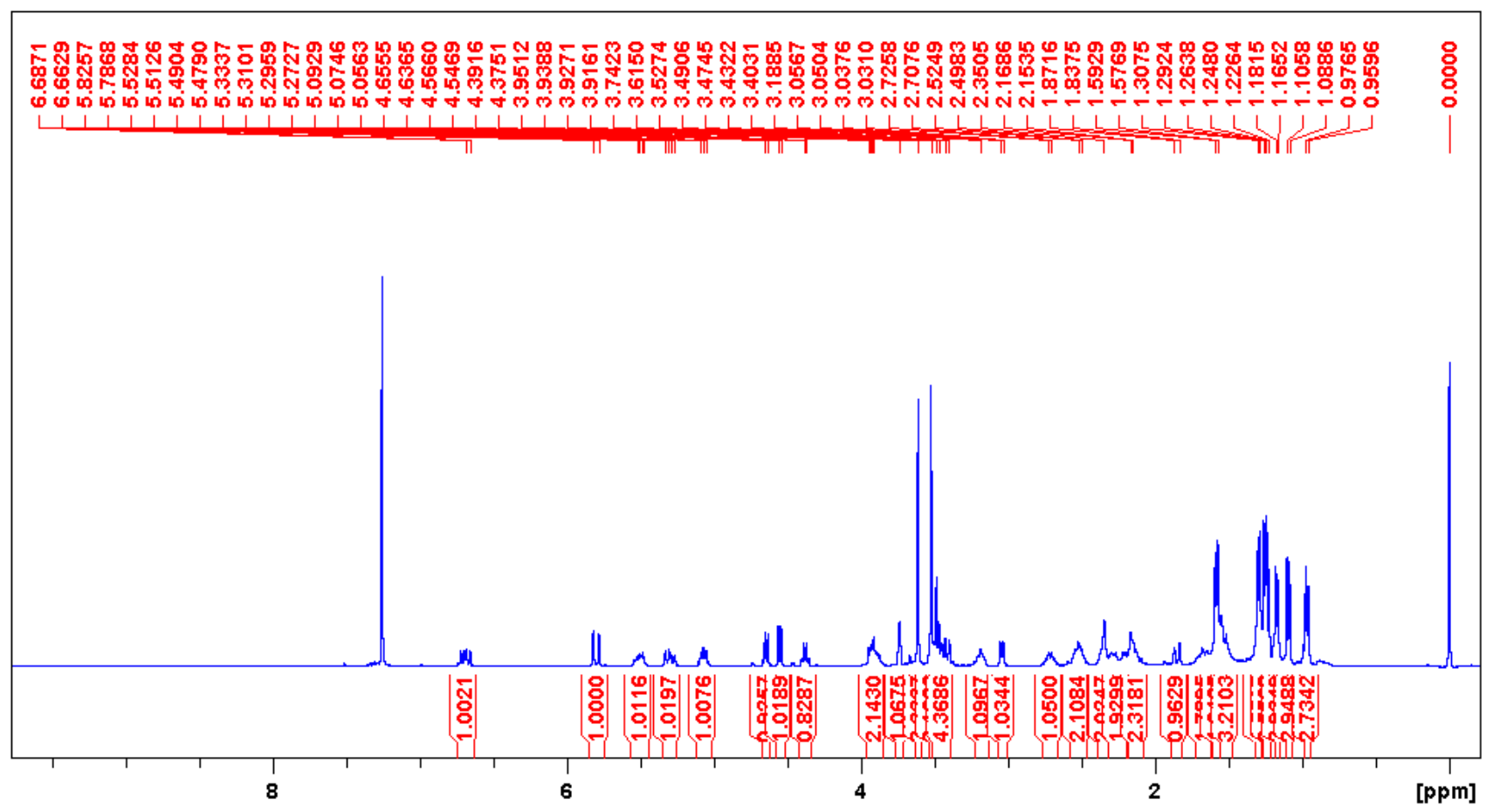

Figure S24. ${ }^{1} \mathrm{H}$ NMR (400 MHz, $\left.\mathrm{CDCl}_{3}\right)$ spectrum for aldgamycin M (4) 


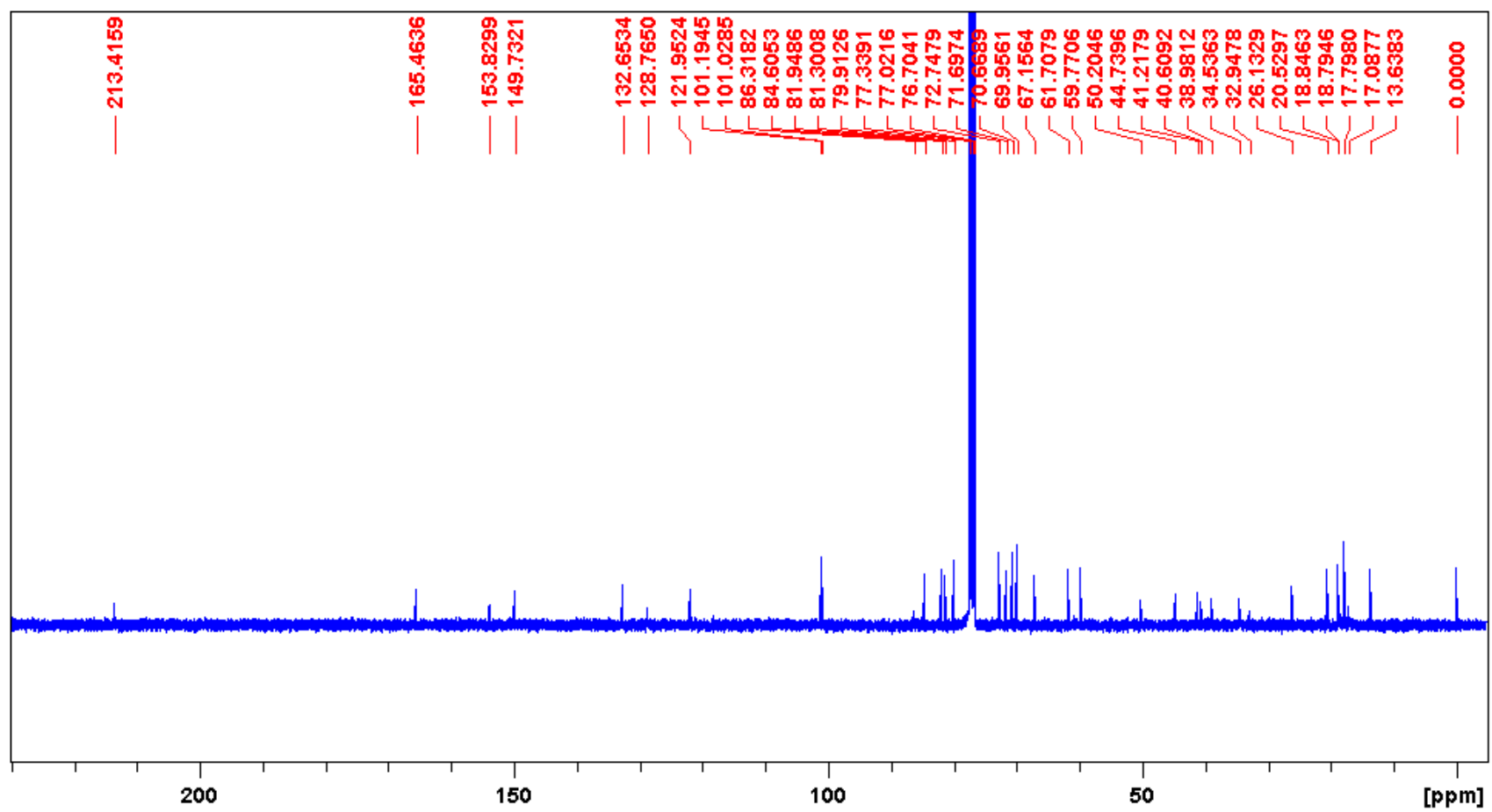

Figure S25. ${ }^{13} \mathrm{C}$ NMR (100 MHz, $\left.\mathrm{CDCl}_{3}\right)$ spectrum for aldgamycin $\mathrm{M}(4)$

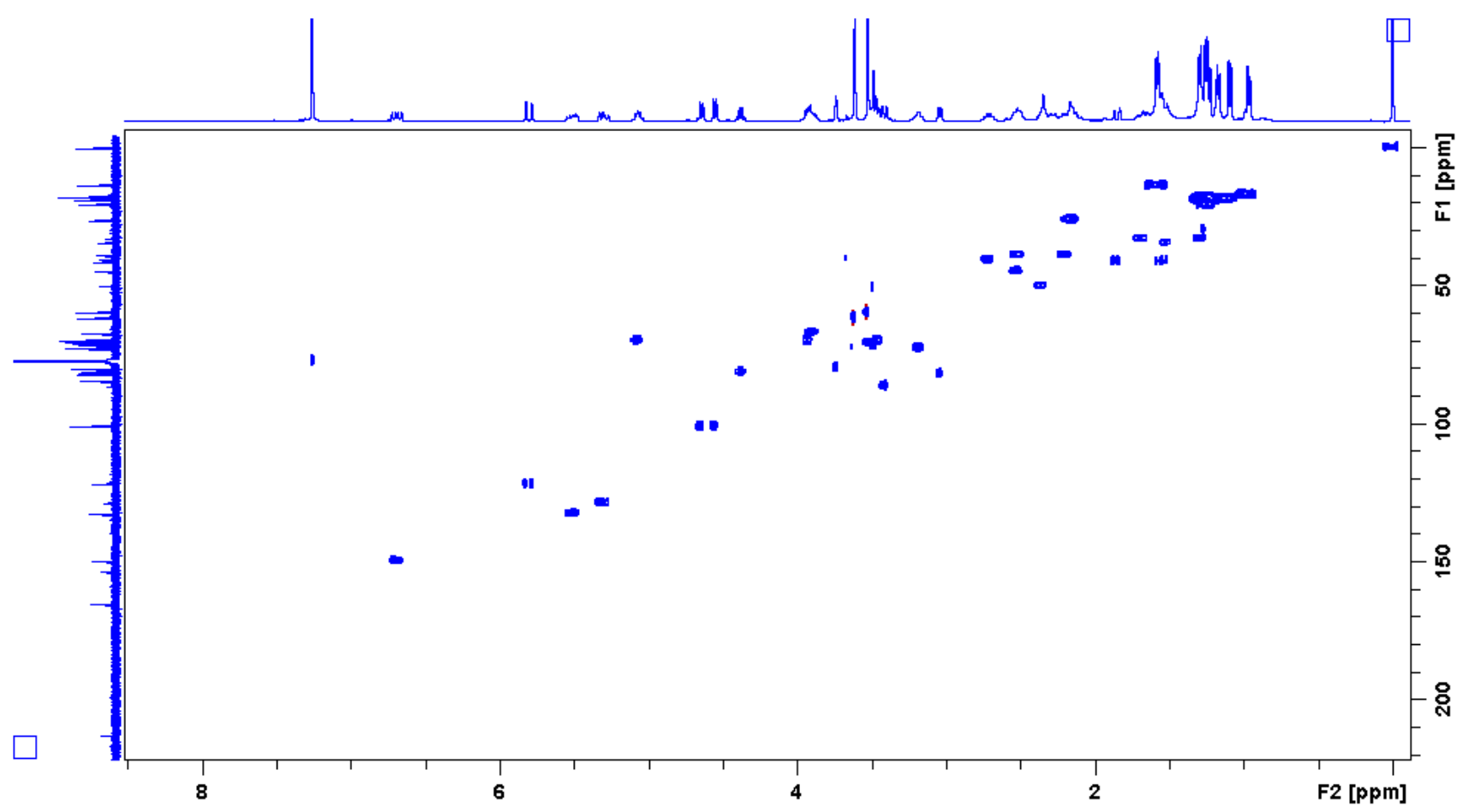

Figure S26. $\mathrm{HSQC}\left(\mathrm{CDCl}_{3}\right)$ spectrum for aldgamycin M (4) 


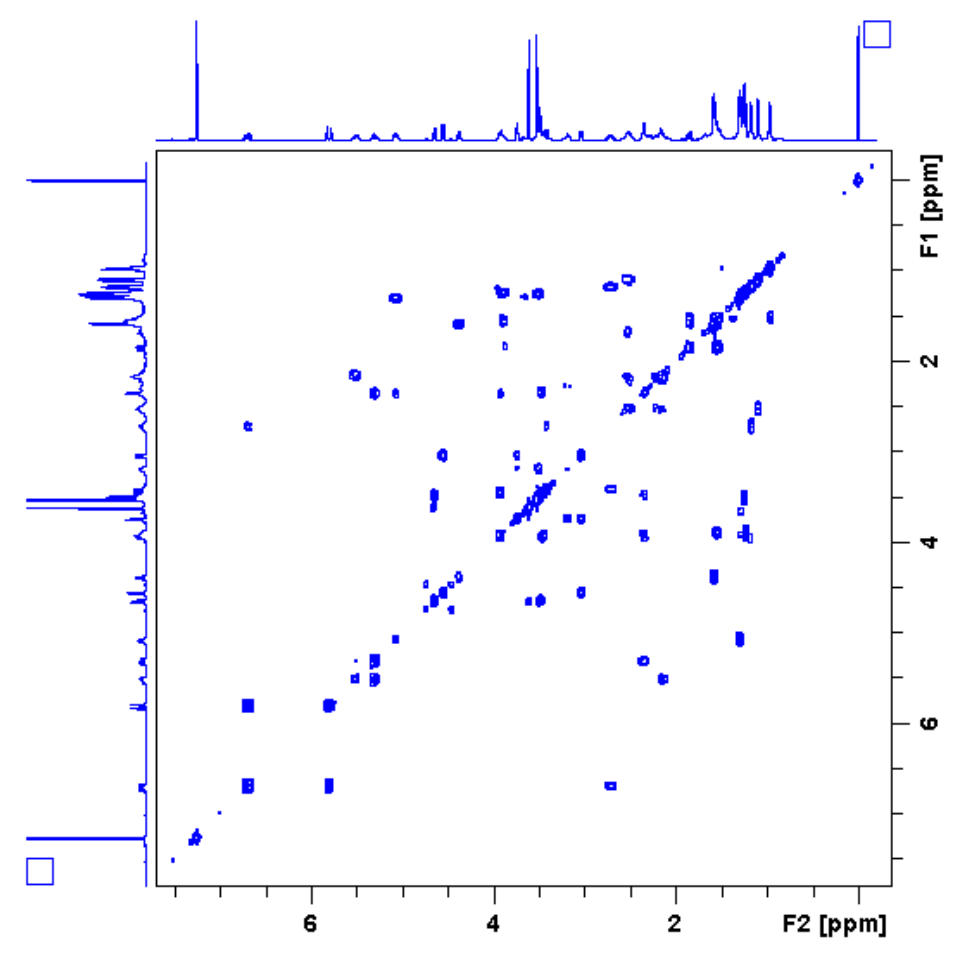

Figure S27. ${ }^{1} \mathrm{H}-{ }^{1} \mathrm{H} \operatorname{COSY}\left(\mathrm{CDCl}_{3}\right)$ spectrum for aldgamycin $\mathrm{M}$ (4)

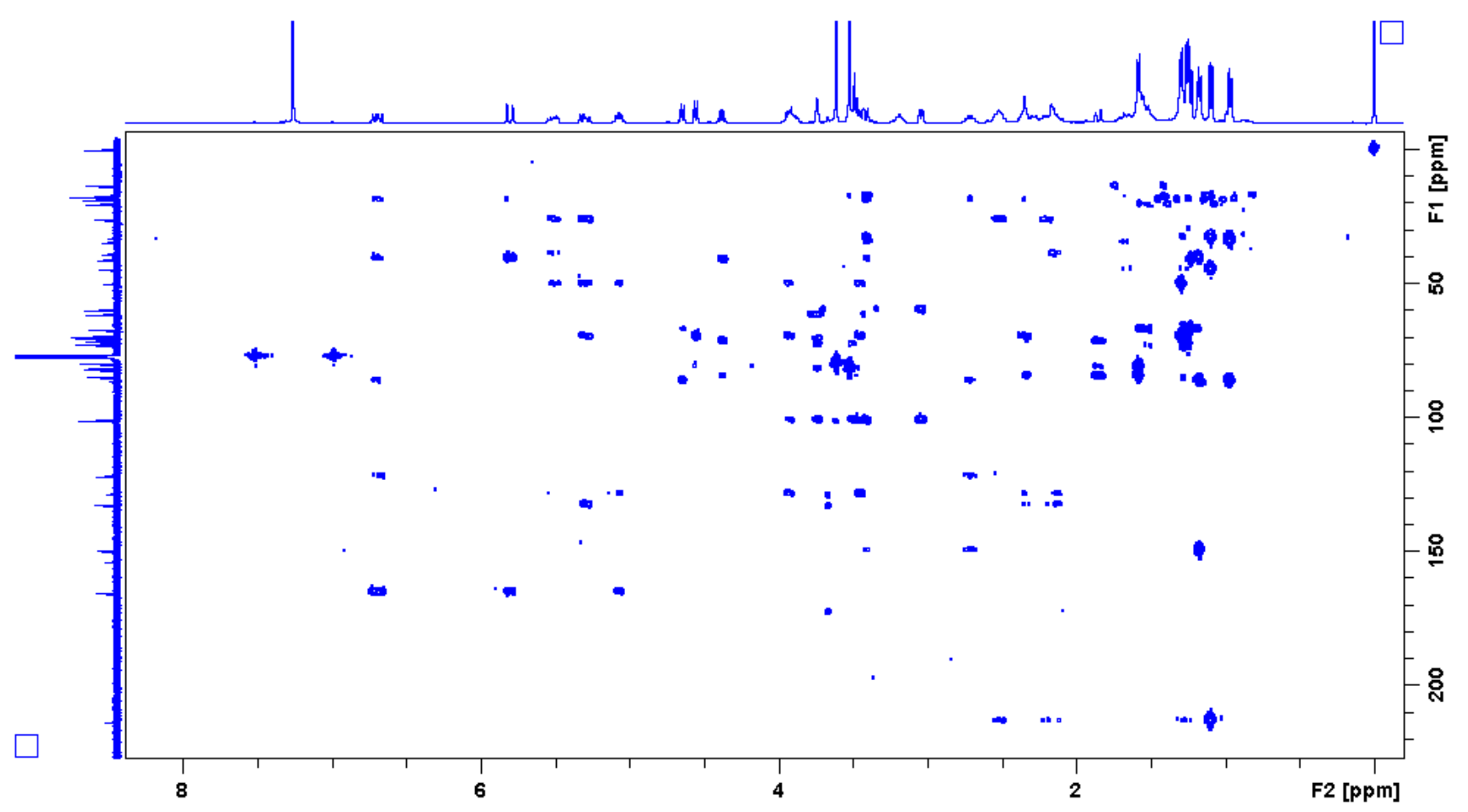

Figure S28. HMBC $\left(\mathrm{CDCl}_{3}\right)$ spectrum for aldgamycin $\mathrm{M}(\mathbf{4})$ 


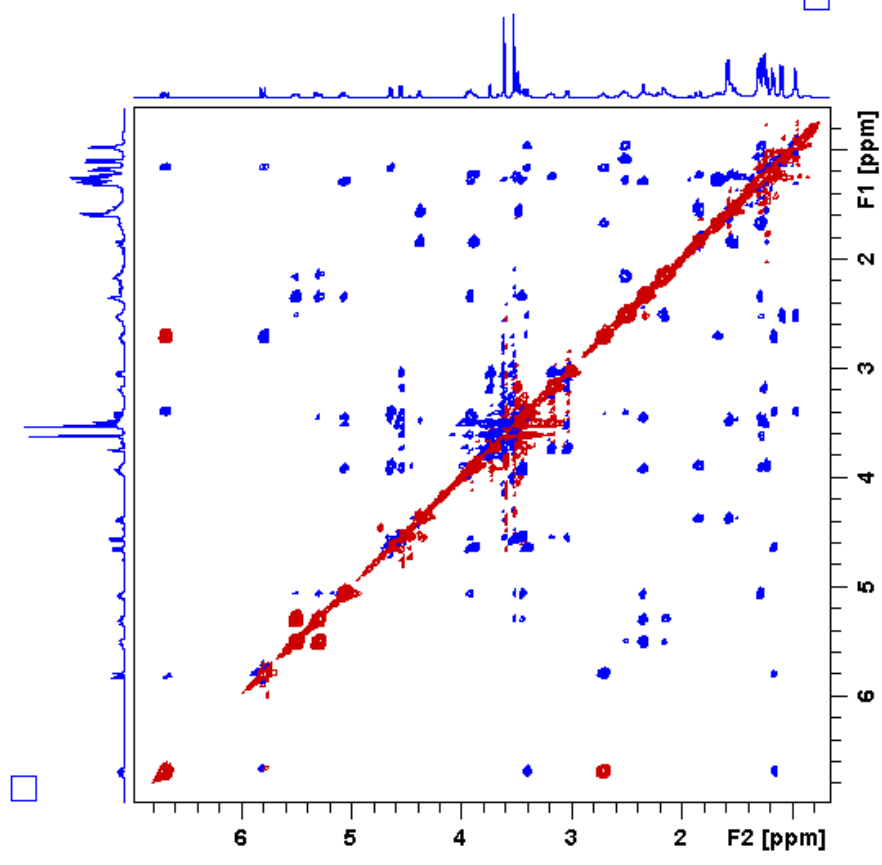

Figure S29. ROESY $\left(\mathrm{CDCl}_{3}\right)$ spectrum for aldgamycin M (4)

The 1D and 2D NMR spectra of aldgamycin N (5)

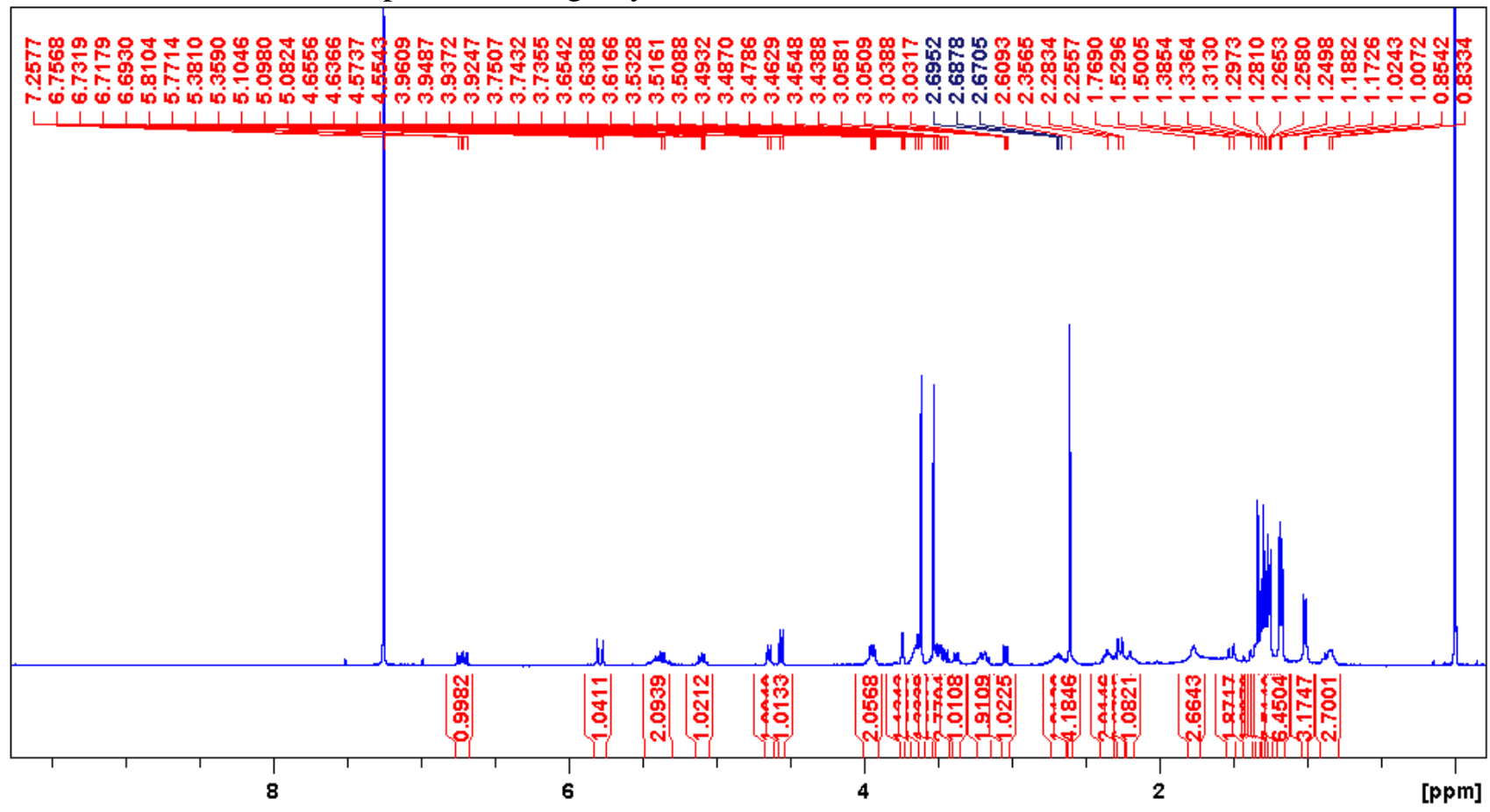

Figure S30. ${ }^{1} \mathrm{H}$ NMR $\left(400 \mathrm{MHz}, \mathrm{CDCl}_{3}\right)$ spectrum for aldgamycin N (5) 


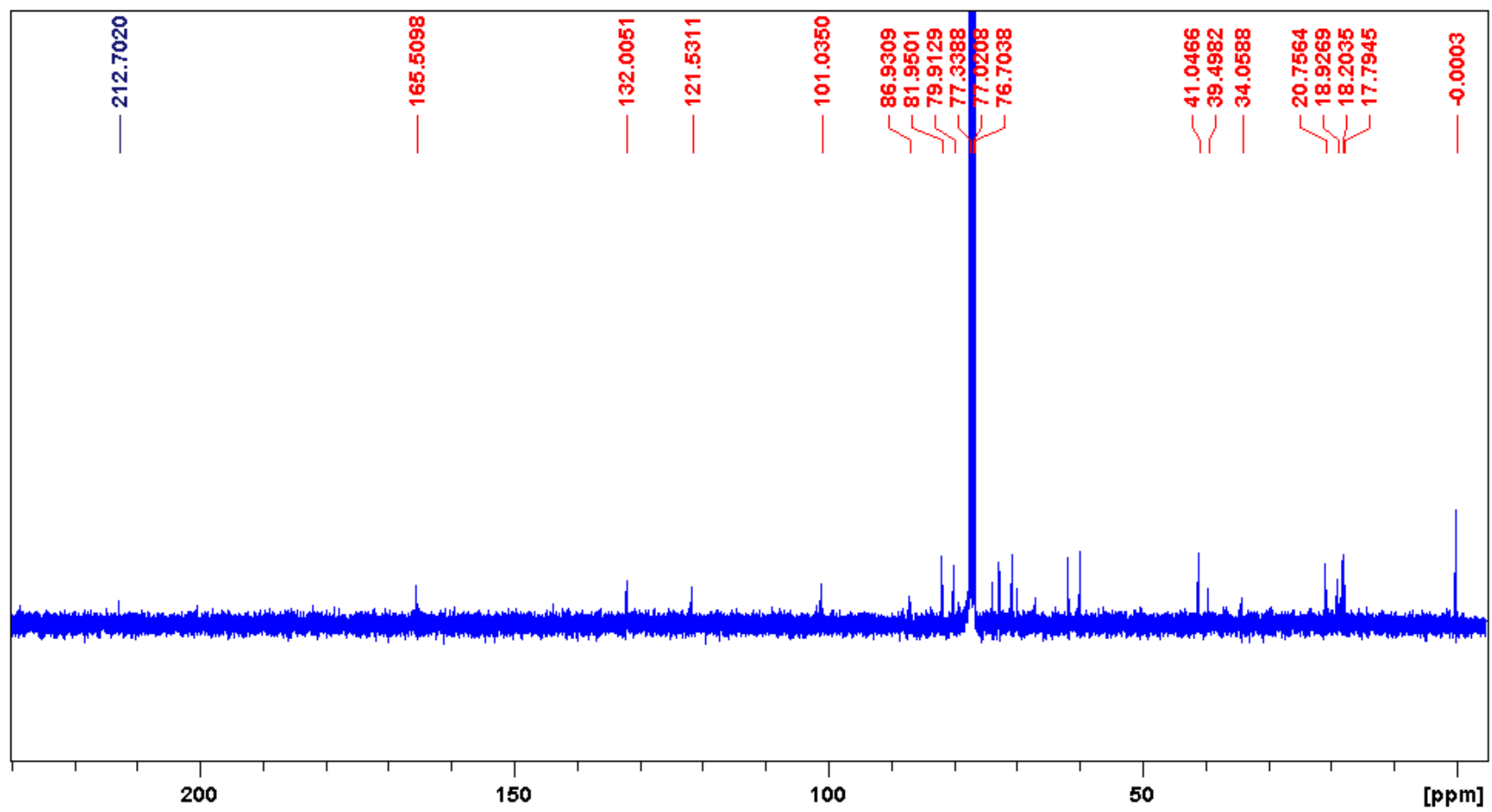

Figure S31. ${ }^{13} \mathrm{C} \mathrm{NMR}\left(100 \mathrm{MHz}, \mathrm{CDCl}_{3}\right)$ spectrum for aldgamycin $\mathrm{N}(\mathbf{5})$

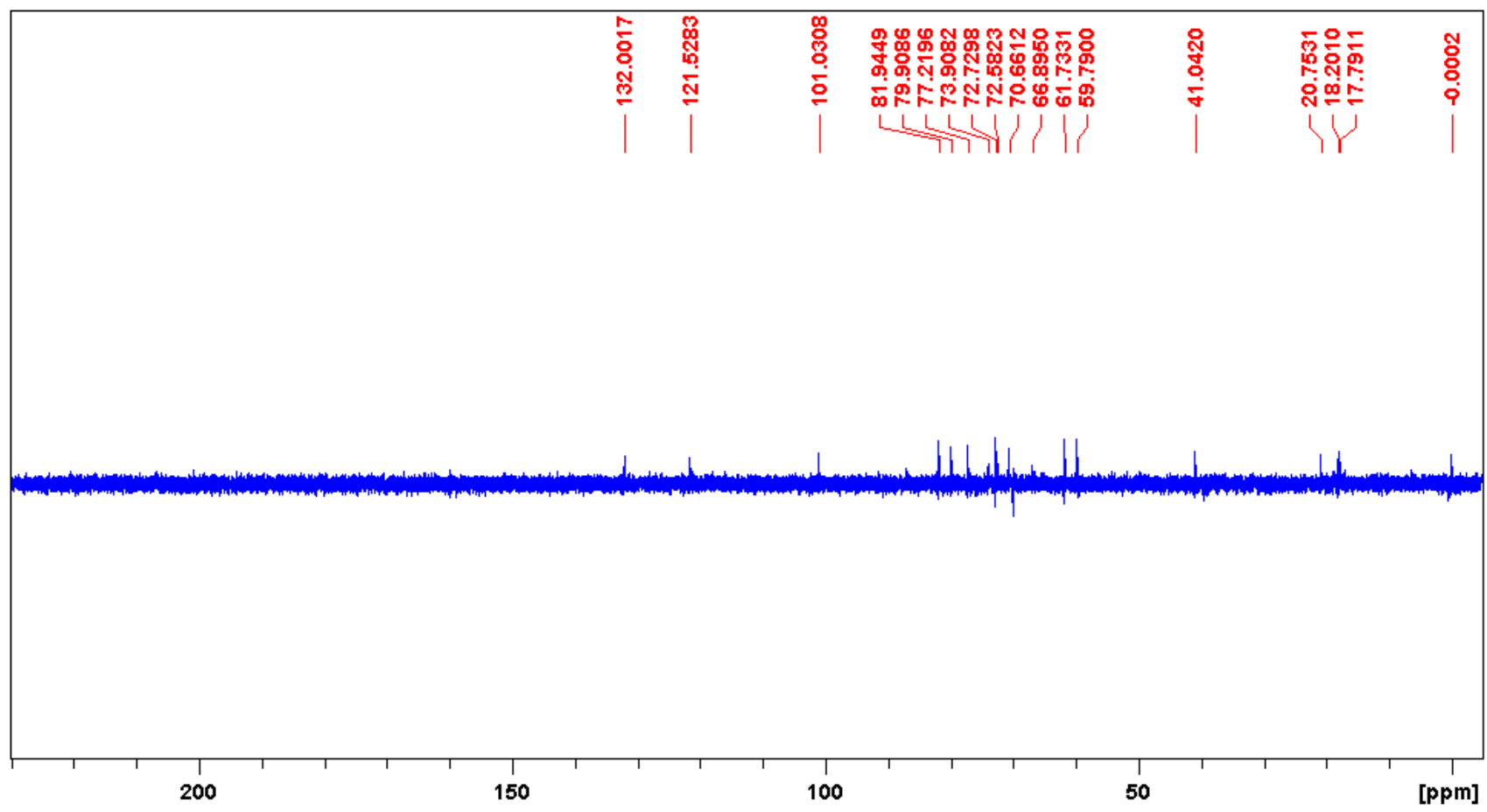

Figure S32. DEPT135 (100 MHz, $\left.\mathrm{CDCl}_{3}\right)$ spectrum for aldgamycin $\mathrm{N}(\mathbf{5})$ 


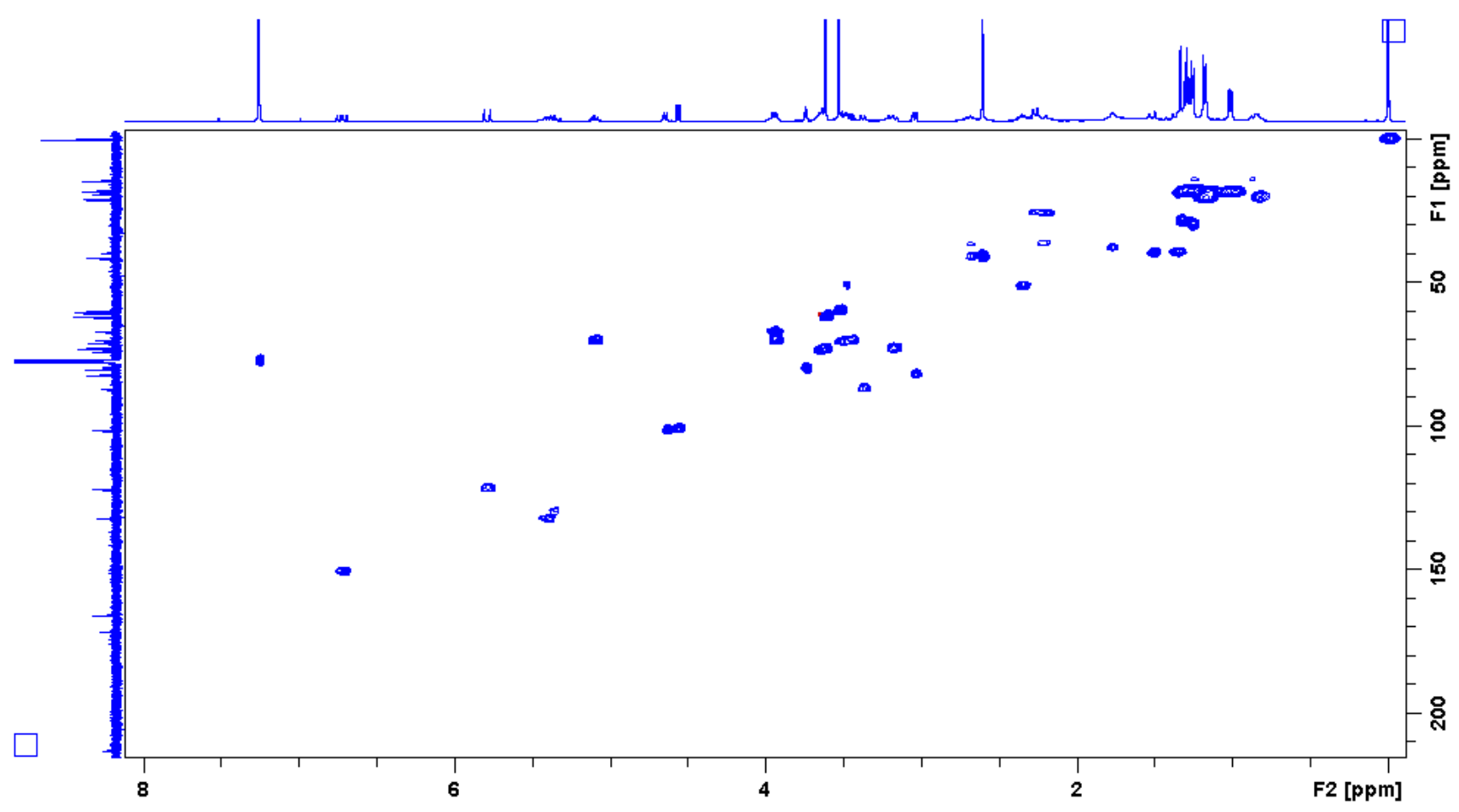

Figure S33. $\mathrm{HSQC}\left(\mathrm{CDCl}_{3}\right)$ spectrum for aldgamycin $\mathrm{N}(\mathbf{5})$

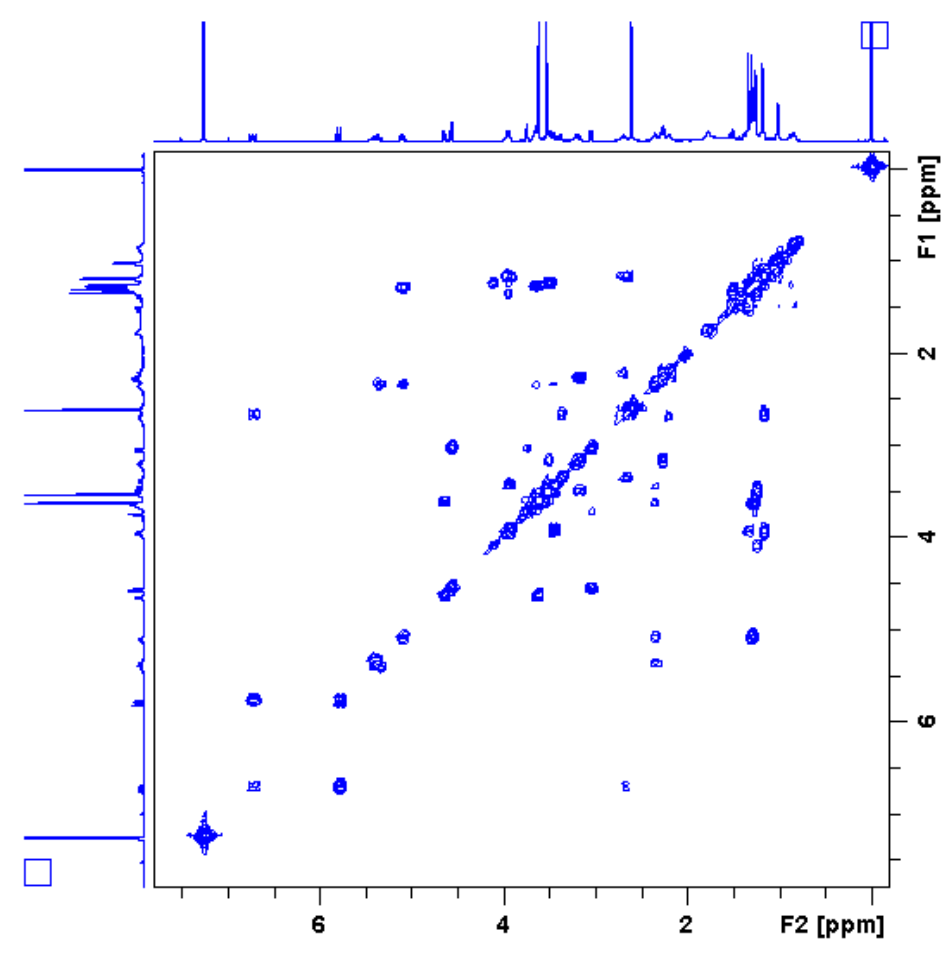

Figure S34. ${ }^{1} \mathrm{H}-{ }^{1} \mathrm{H} \operatorname{COSY}\left(\mathrm{CDCl}_{3}\right)$ spectrum for aldgamycin $\mathrm{N}(\mathbf{5})$ 


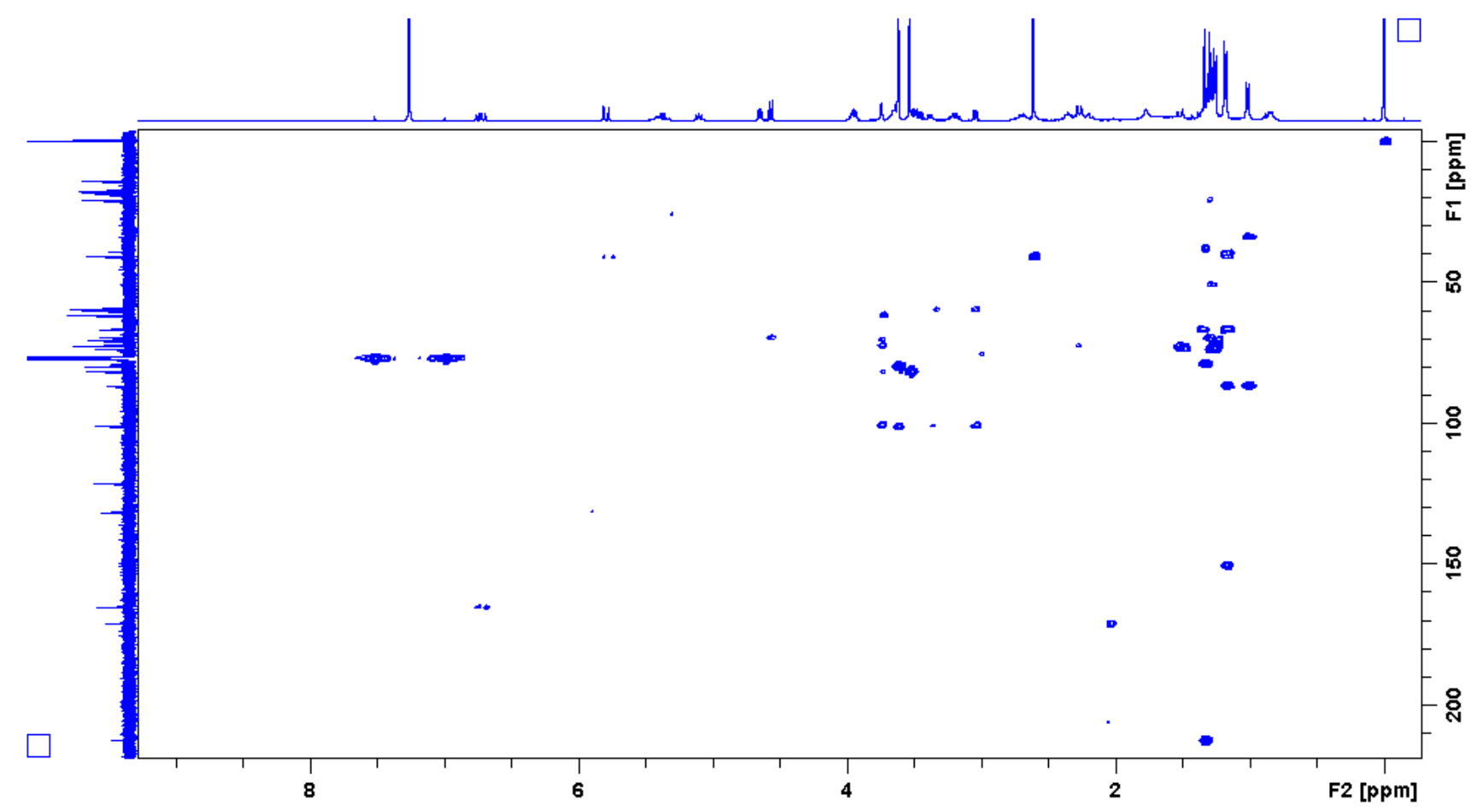

Figure S35. $\mathrm{HMBC}\left(\mathrm{CDCl}_{3}\right)$ spectrum for aldgamycin $\mathrm{N}(\mathbf{5})$

The 1D and 2D NMR spectra of aldgamycin O (6)

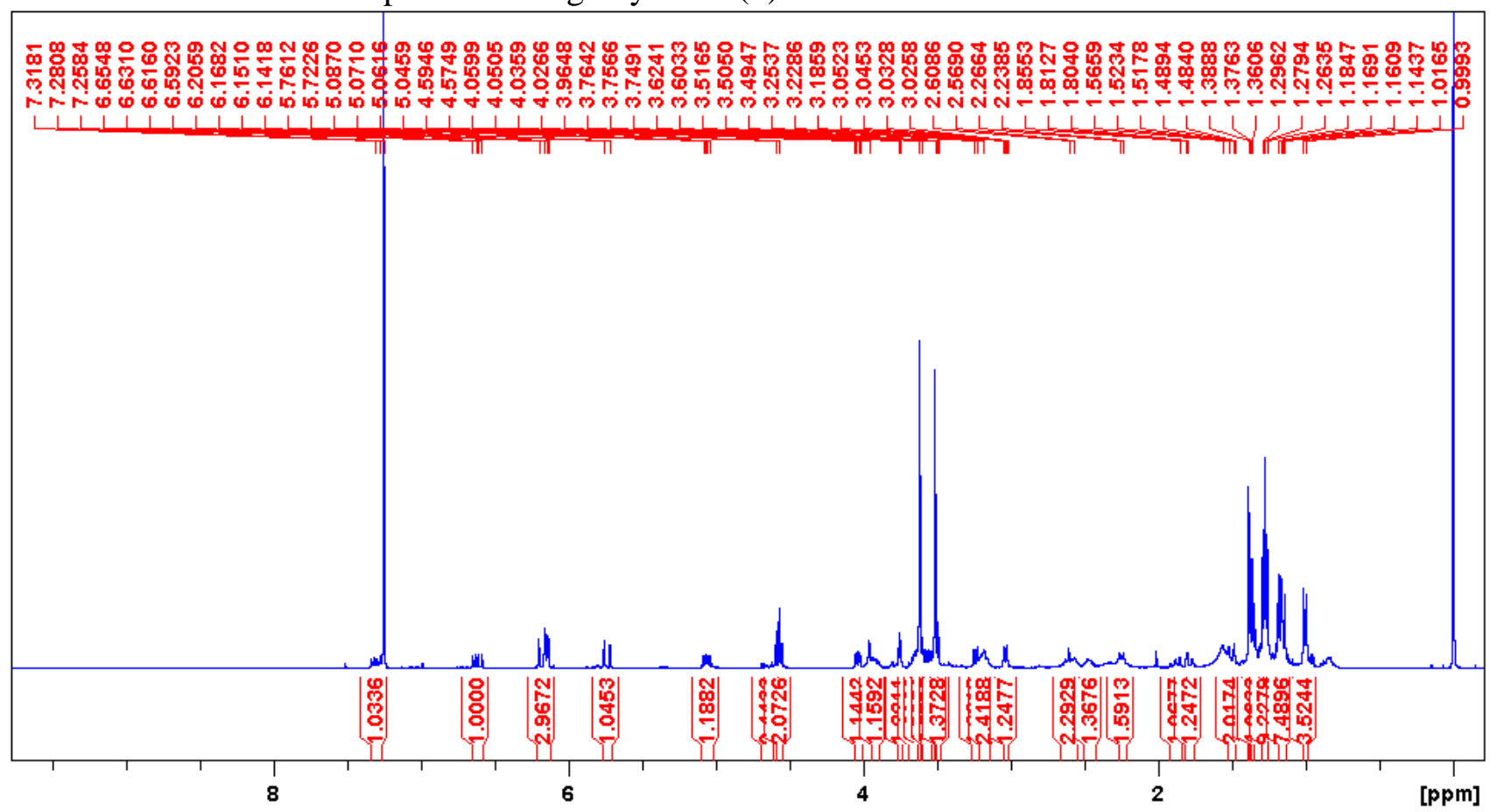

Figure S36. ${ }^{1} \mathrm{H}$ NMR $\left(400 \mathrm{MHz}, \mathrm{CDCl}_{3}\right)$ spectrum for aldgamycin $\mathrm{O}(\mathbf{6})$ 


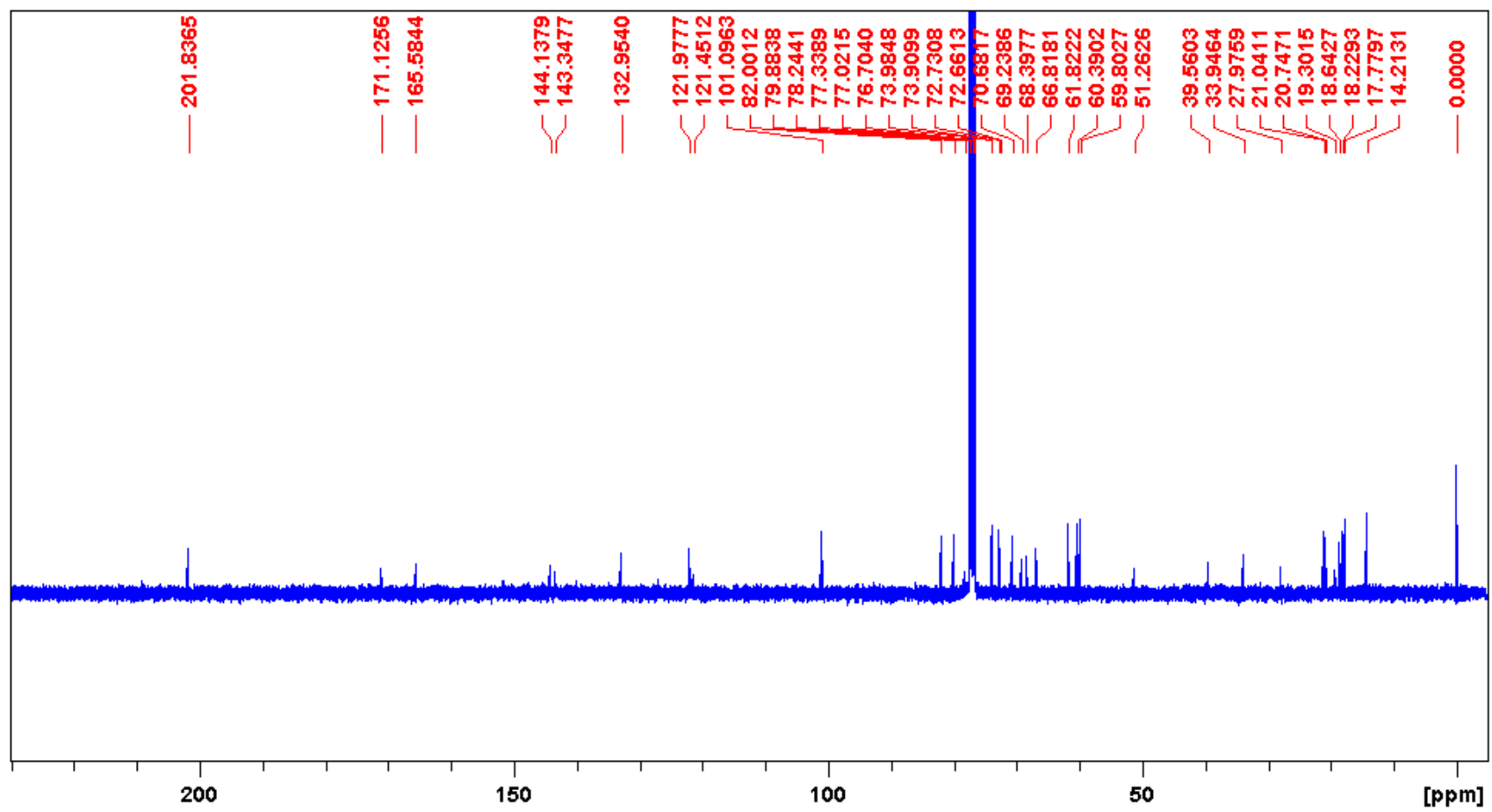

Figure S37. ${ }^{13} \mathrm{C} \mathrm{NMR}\left(100 \mathrm{MHz}, \mathrm{CDCl}_{3}\right)$ spectrum for aldgamycin $\mathrm{O}(\mathbf{6})$

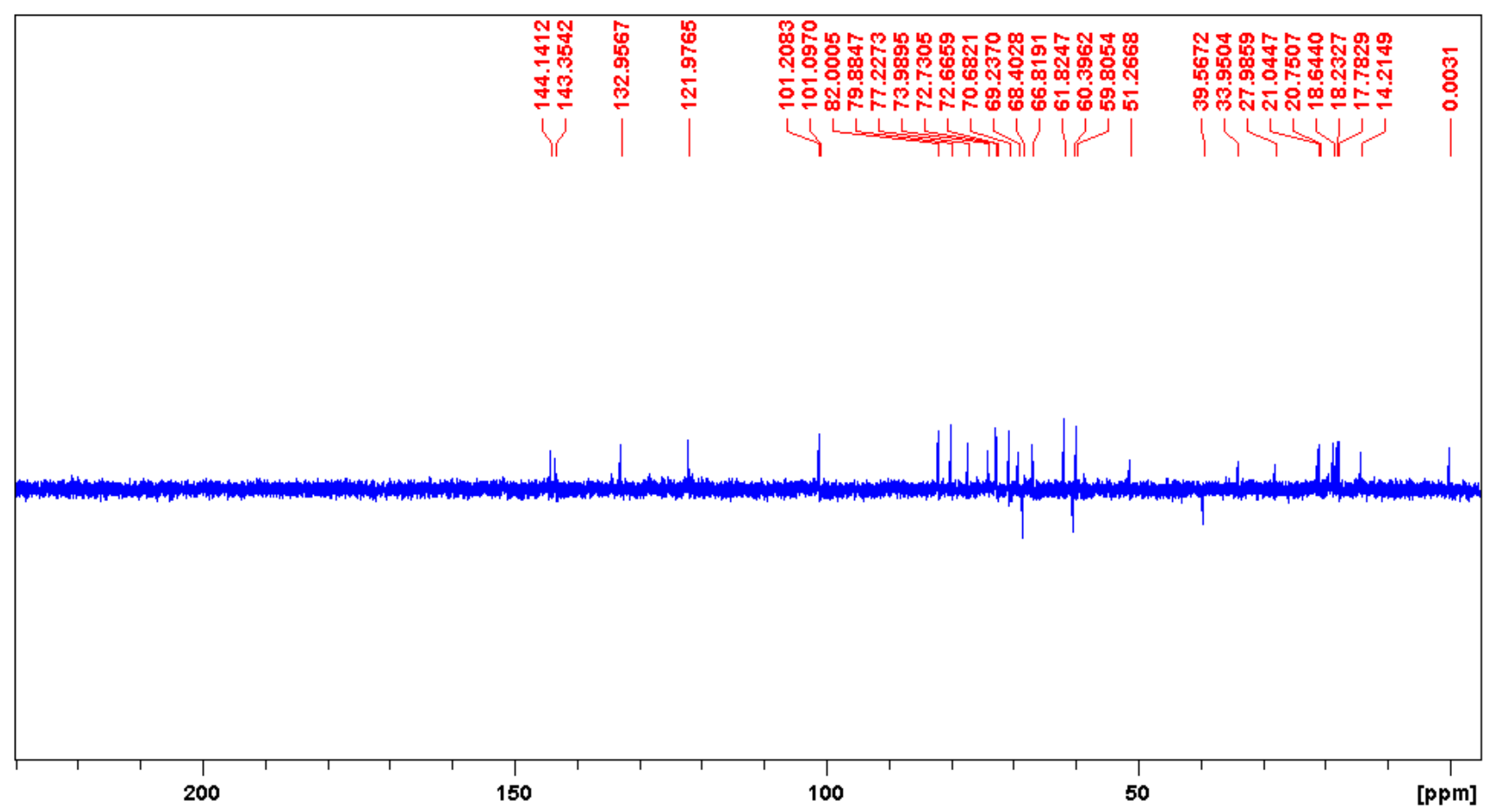

Figure S38. DEPT135 (100 MHz, $\left.\mathrm{CDCl}_{3}\right)$ spectrum for aldgamycin $\mathrm{O}(\mathbf{6})$ 


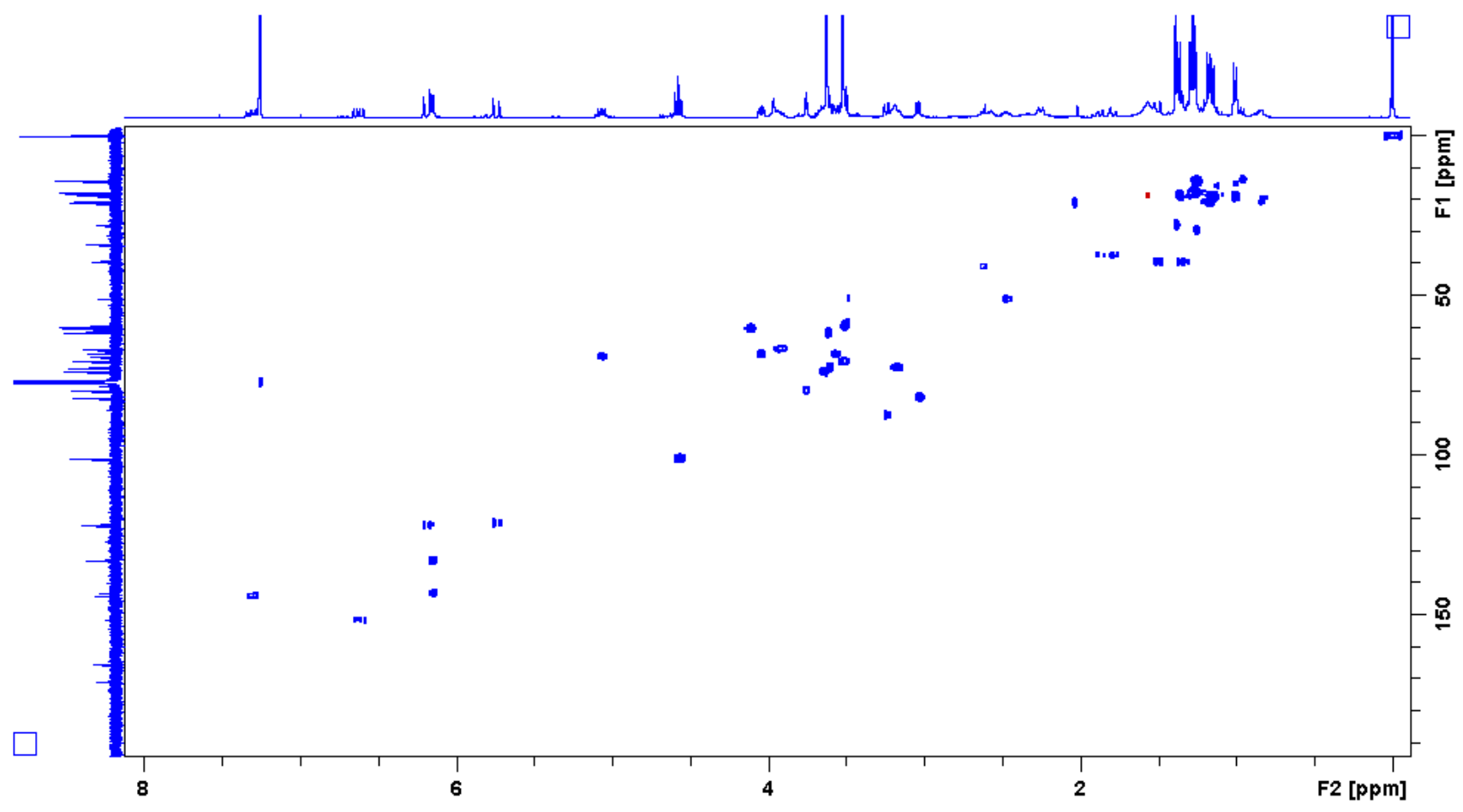

Figure S39. HSQC $\left(\mathrm{CDCl}_{3}\right)$ spectrum for aldgamycin $\mathrm{O}(\mathbf{6})$

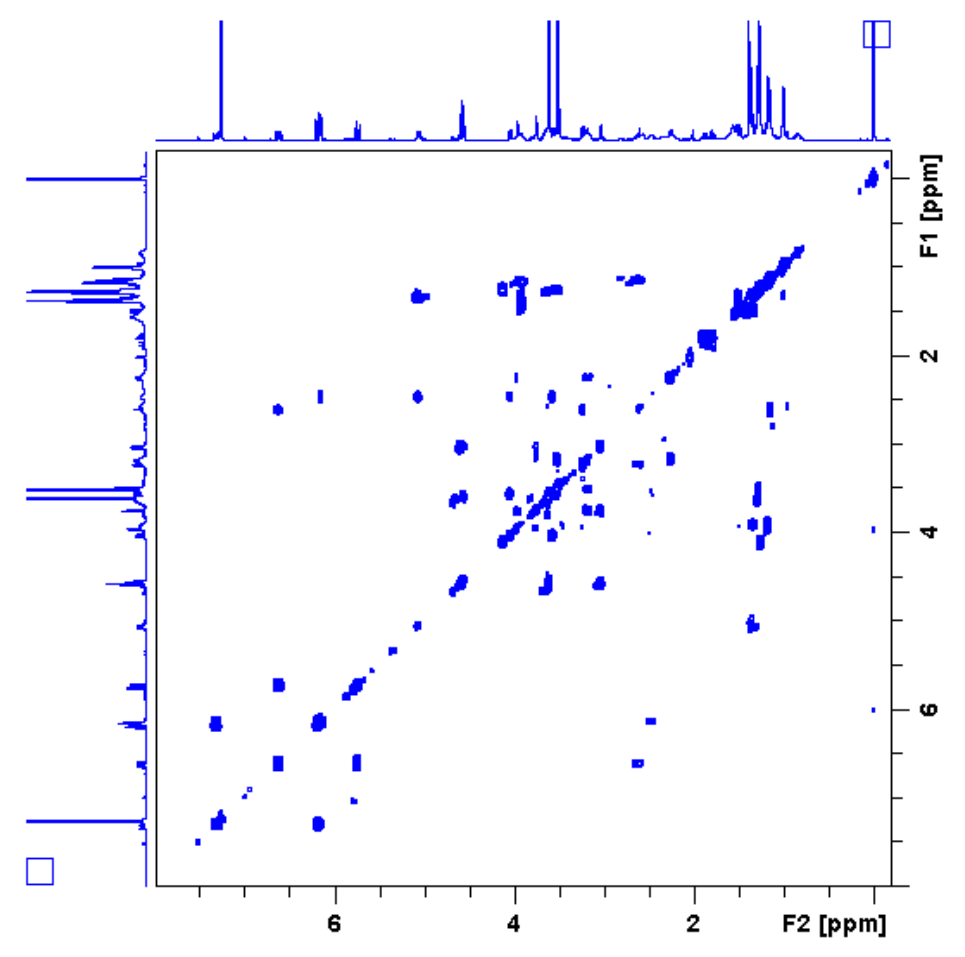

Figure S40. ${ }^{1} \mathrm{H}-{ }^{1} \mathrm{H} \operatorname{COSY}\left(\mathrm{CDCl}_{3}\right)$ spectrum for aldgamycin $\mathrm{O}(\mathbf{6})$ 


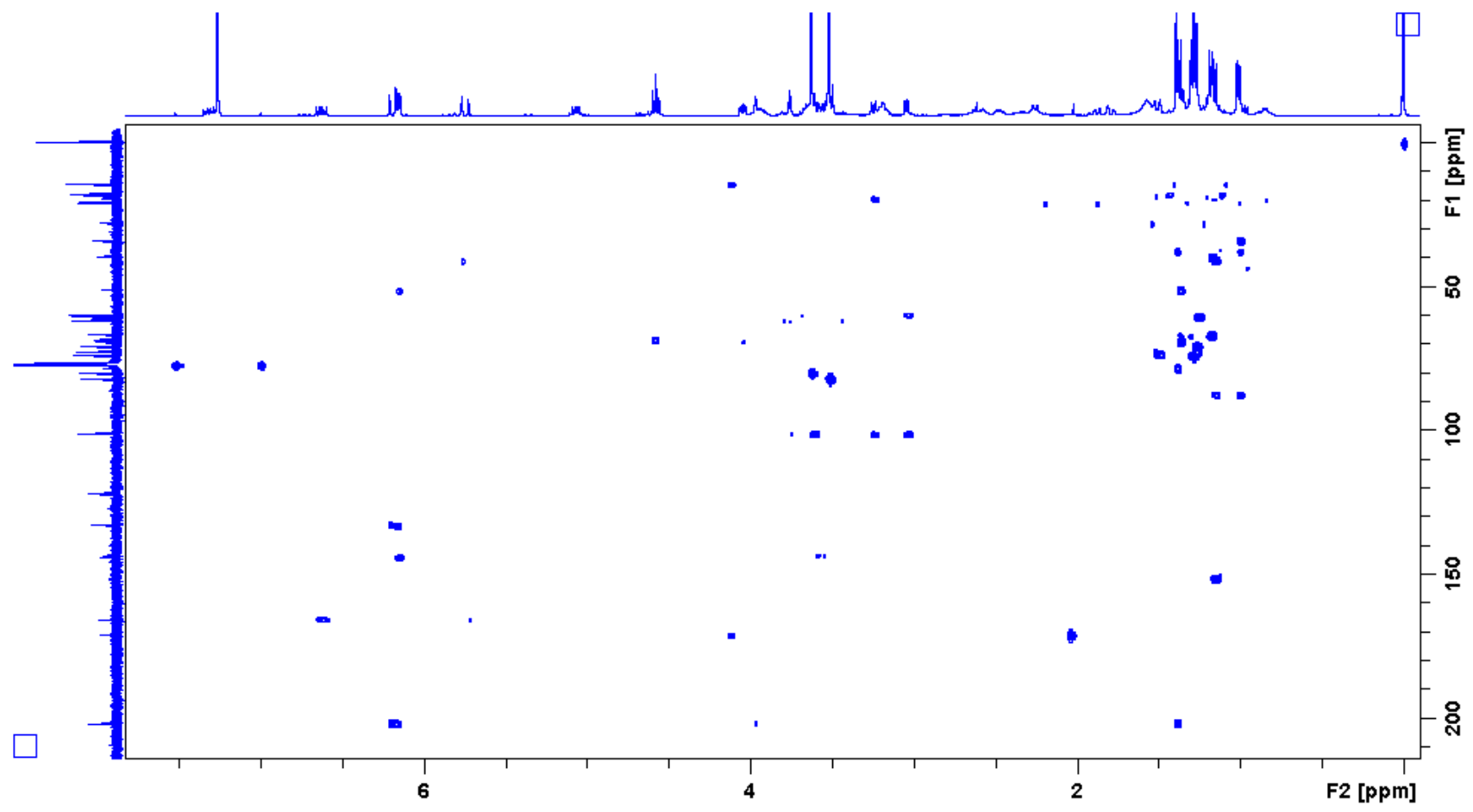

Figure S41. $\mathrm{HMBC}\left(\mathrm{CDCl}_{3}\right)$ spectrum for aldgamycin O (6)

The 1D and 2D NMR spectra of swalpamycin B (7)

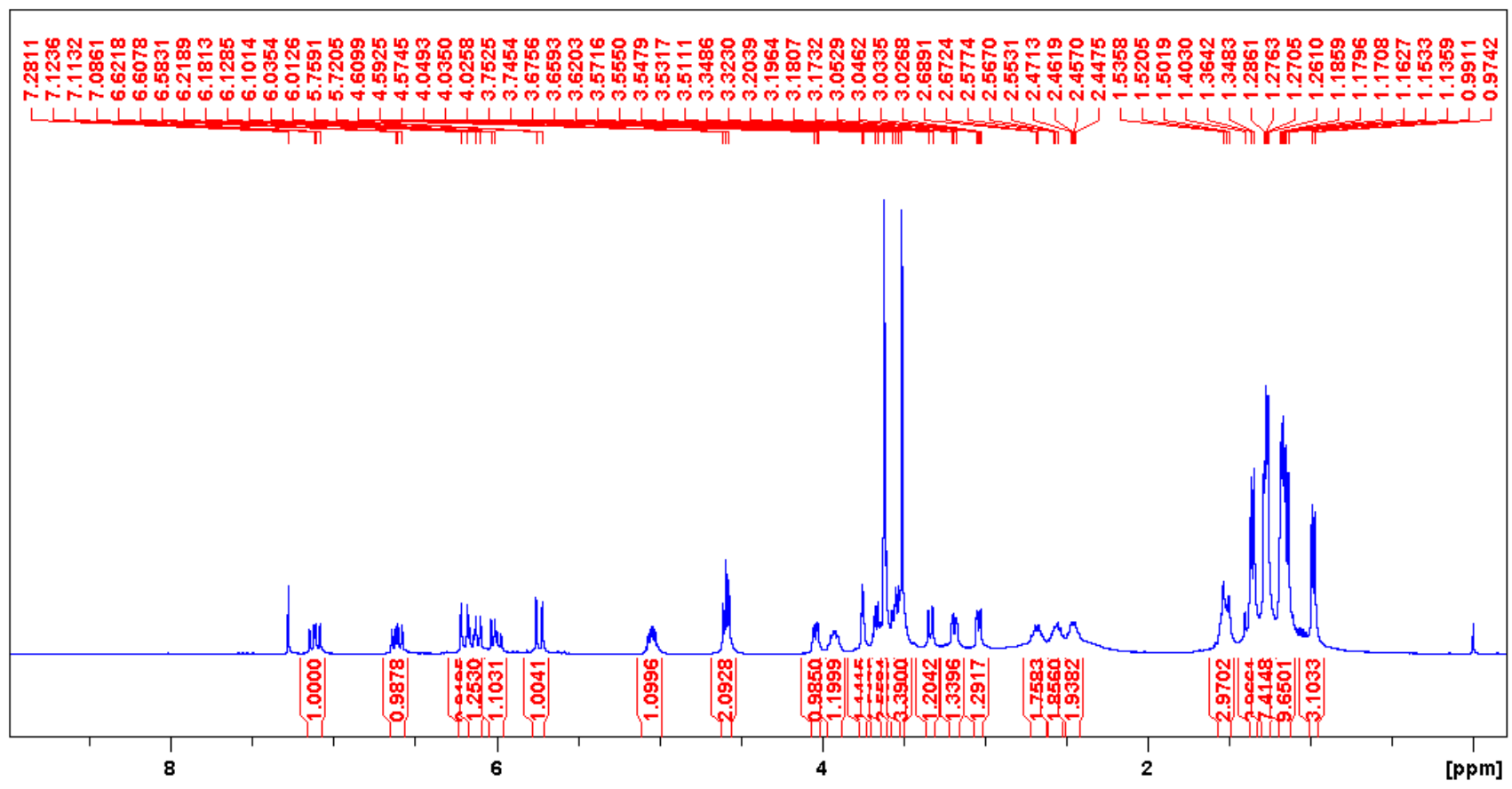

Figure S42. ${ }^{1} \mathrm{H}$ NMR $\left(400 \mathrm{MHz}, \mathrm{CDCl}_{3}\right)$ spectrum for swalpamycin B (7) 


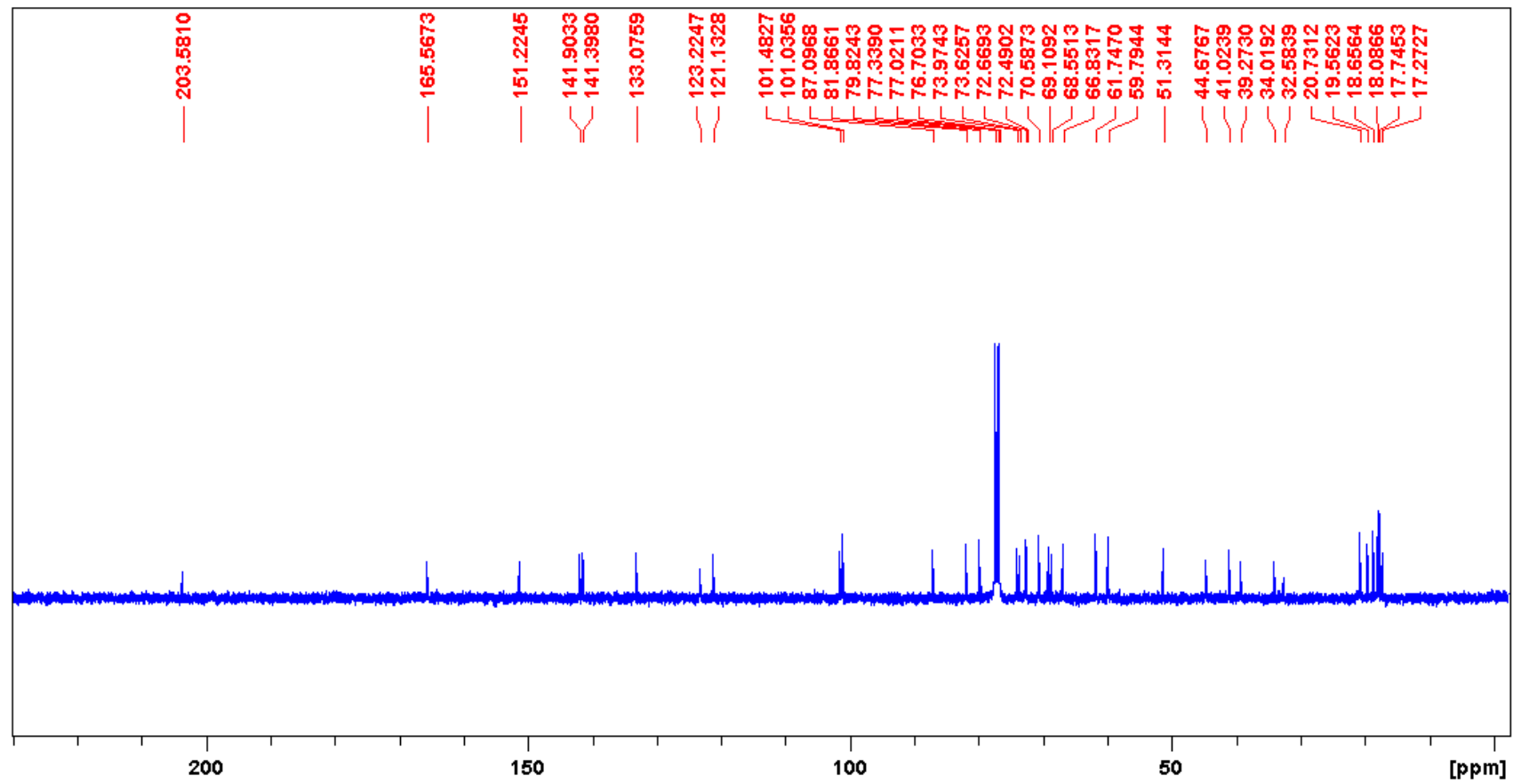

Figure S43. ${ }^{13} \mathrm{C}$ NMR $\left(100 \mathrm{MHz}, \mathrm{CDCl}_{3}\right)$ spectrum for swalpamycin B (7)

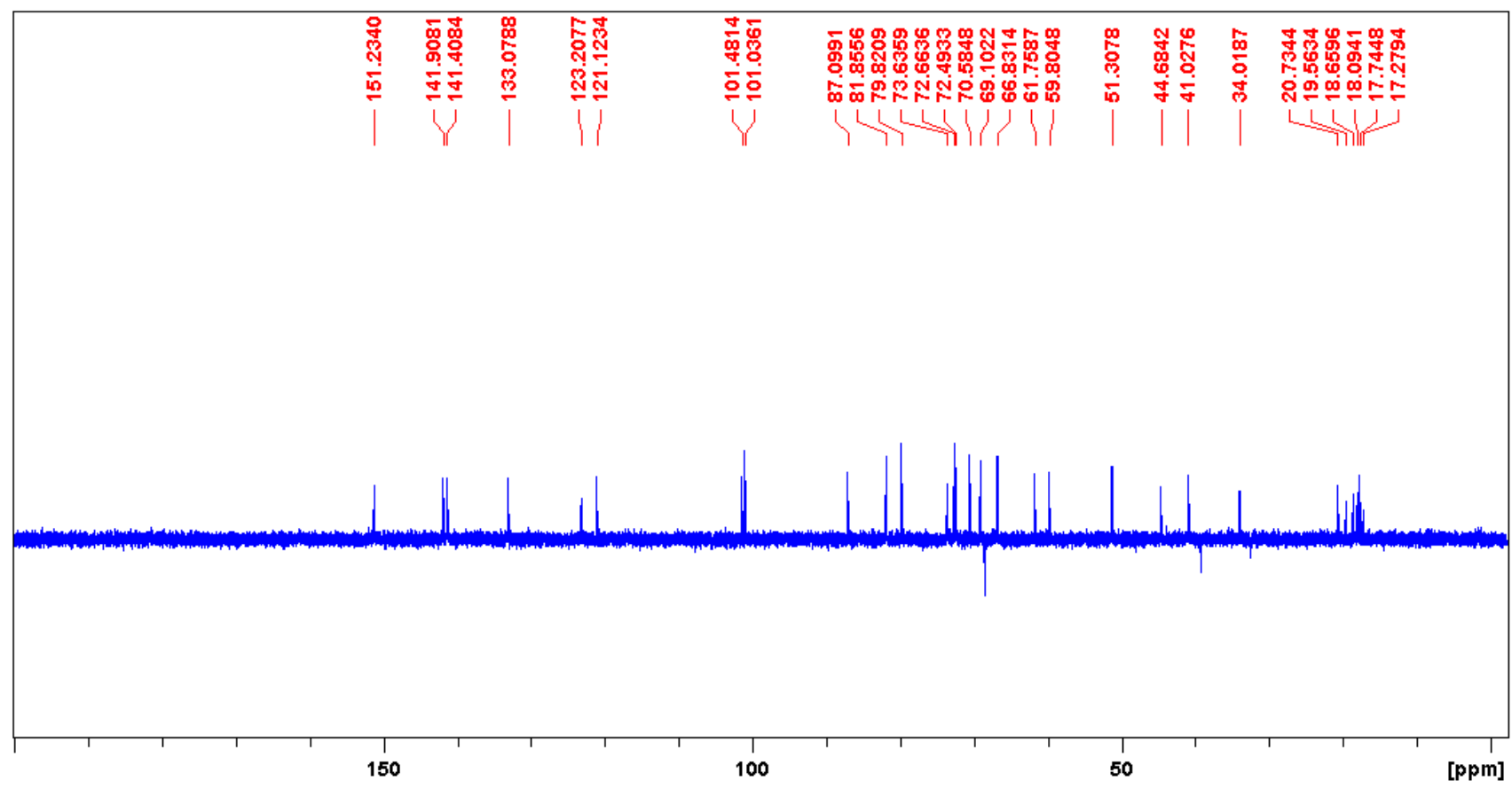

Figure S44. DEPT135 (100 MHz, $\left.\mathrm{CDCl}_{3}\right)$ spectrum for swalpamycin B (7) 


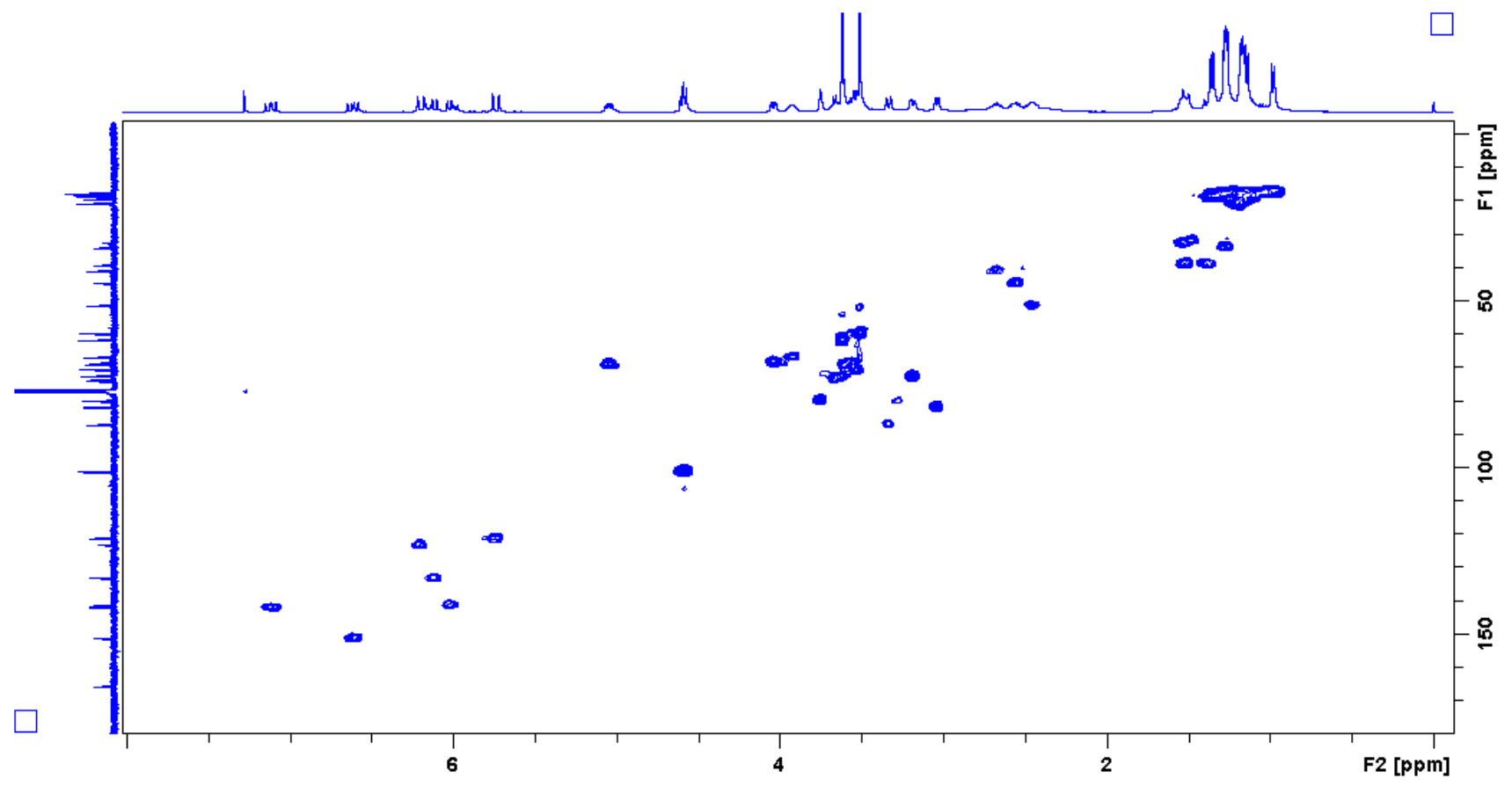

Figure S45. HSQC $\left(\mathrm{CDCl}_{3}\right)$ spectrum for swalpamycin B (7)

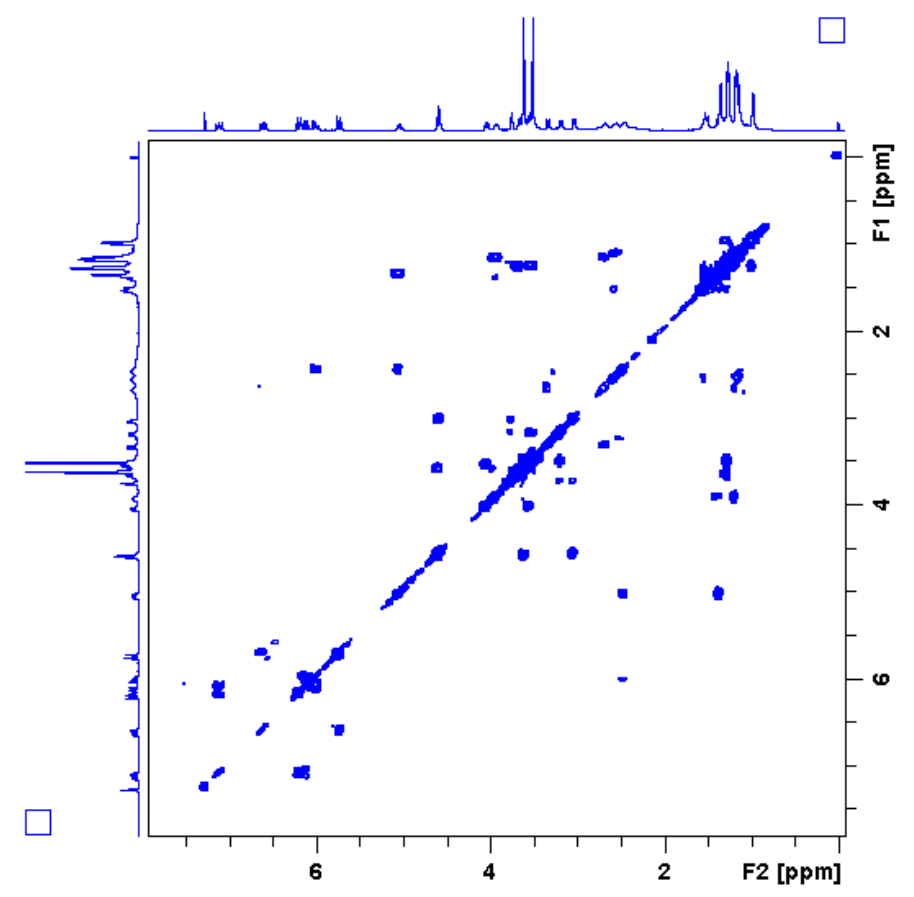

Figure S46. ${ }^{1} \mathrm{H}-{ }^{1} \mathrm{H} \operatorname{COSY}\left(\mathrm{CDCl}_{3}\right)$ spectrum for swalpamycin B (7) 


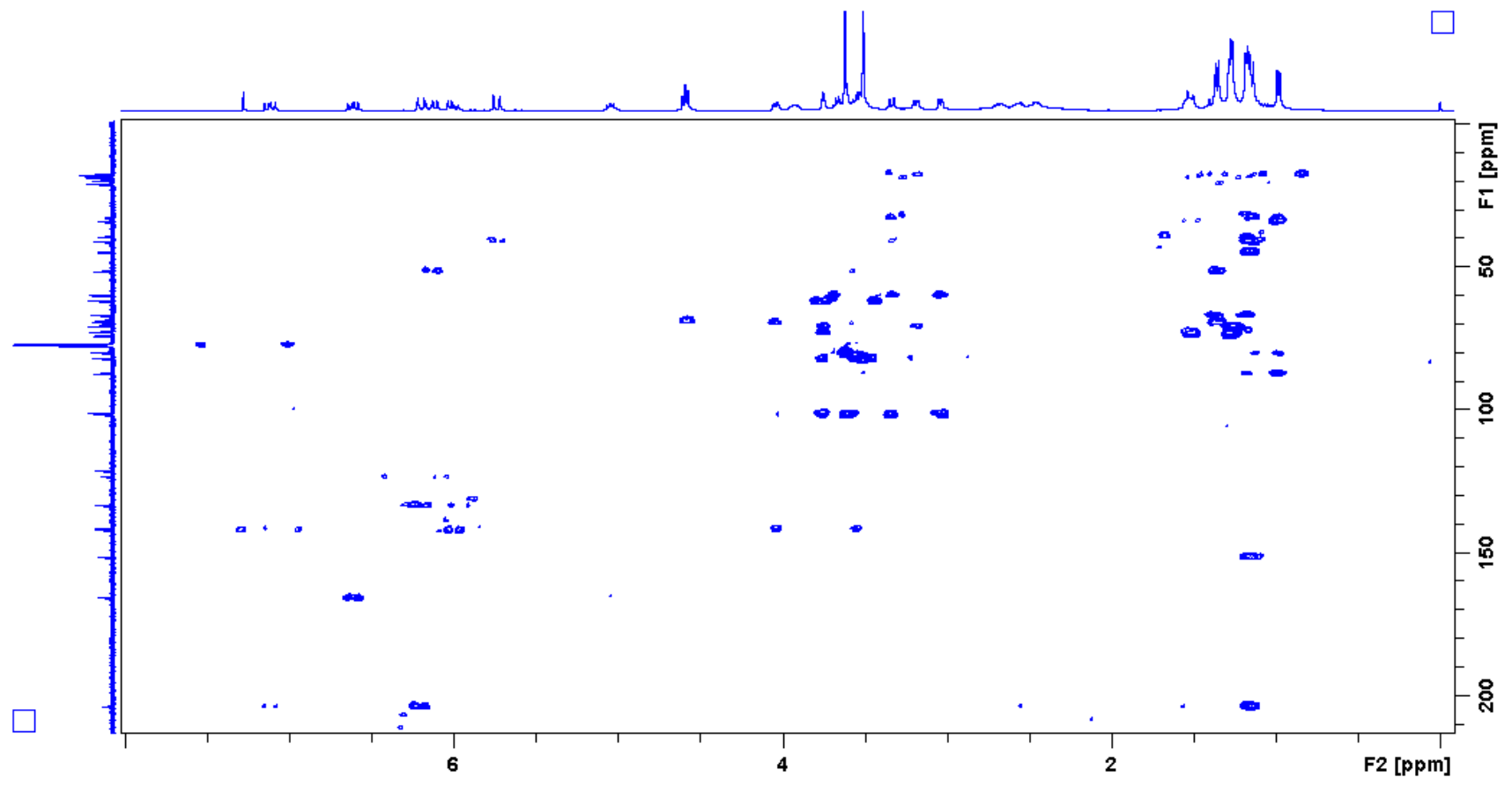

Figure S47. $\mathrm{HMBC}\left(\mathrm{CDCl}_{3}\right)$ spectrum for swalpamycin B (7)

The 1D and 2D NMR spectra of chalcomycin (8)

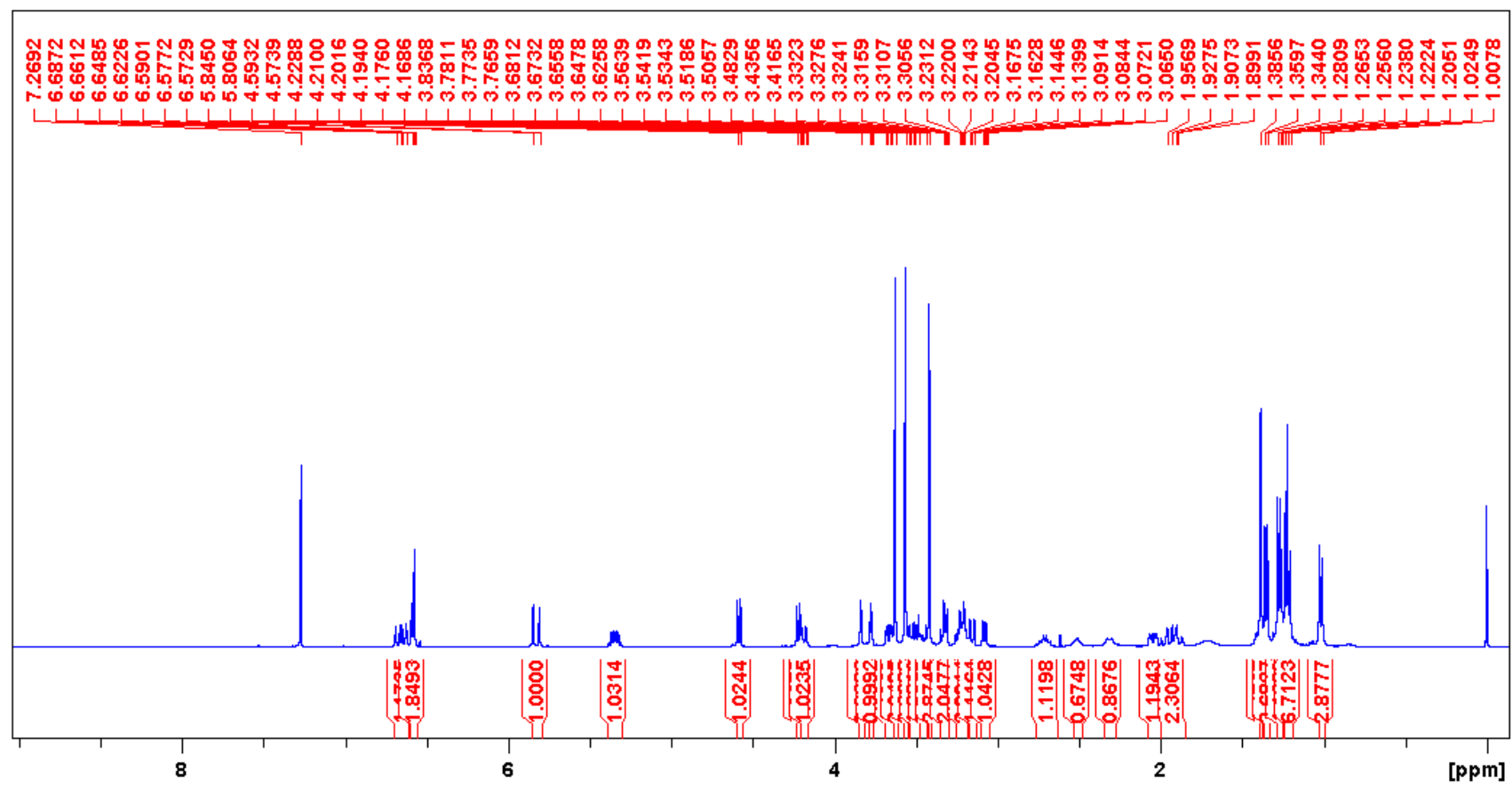

Figure S48. ${ }^{1} \mathrm{H}$ NMR $\left(400 \mathrm{MHz}, \mathrm{CDCl}_{3}\right)$ spectrum for chalcomycin (8) 


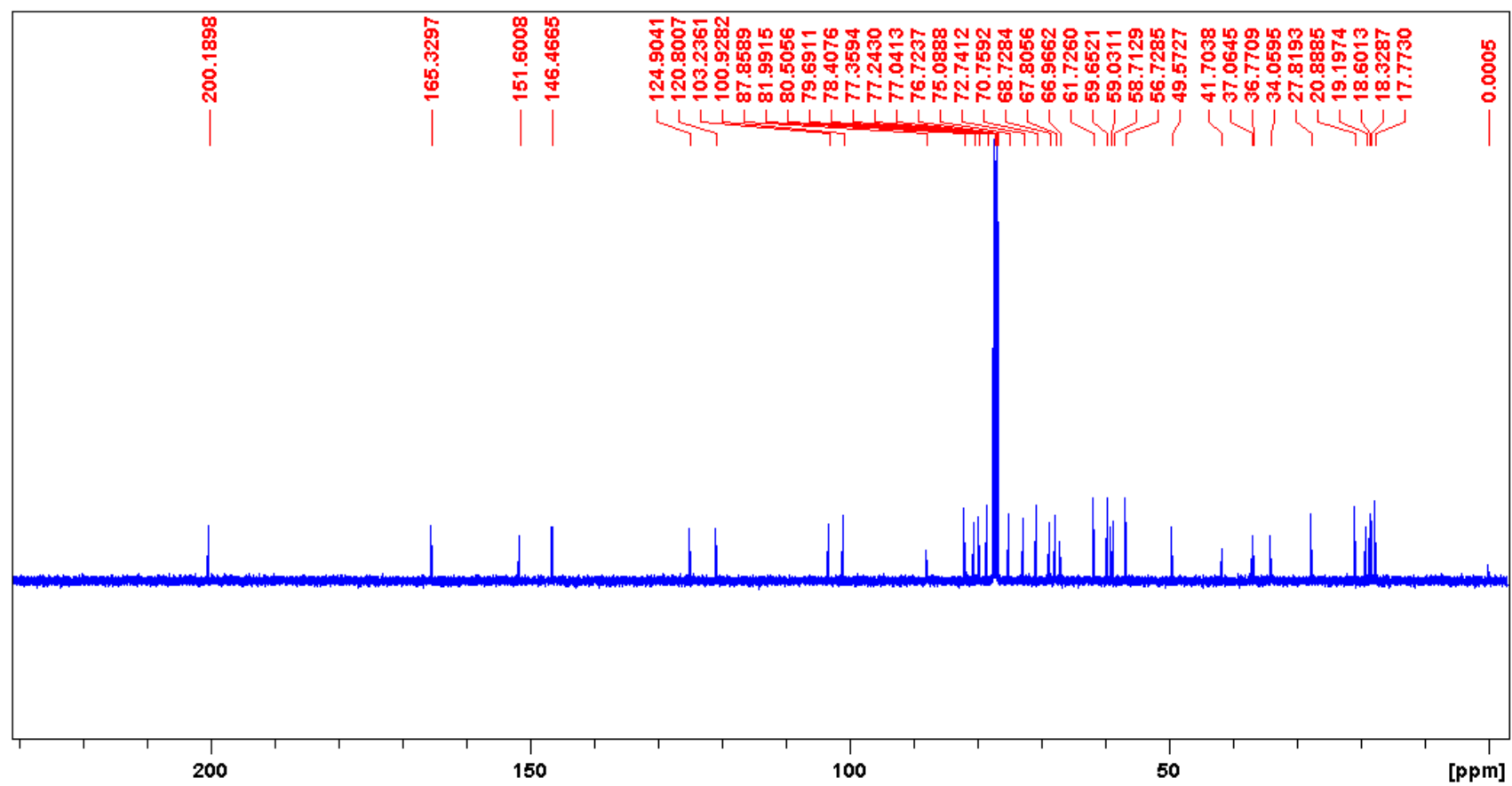

Figure S49. ${ }^{13} \mathrm{C}$ NMR $\left(100 \mathrm{MHz}, \mathrm{CDCl}_{3}\right)$ spectrum for chalcomycin $(\mathbf{8})$

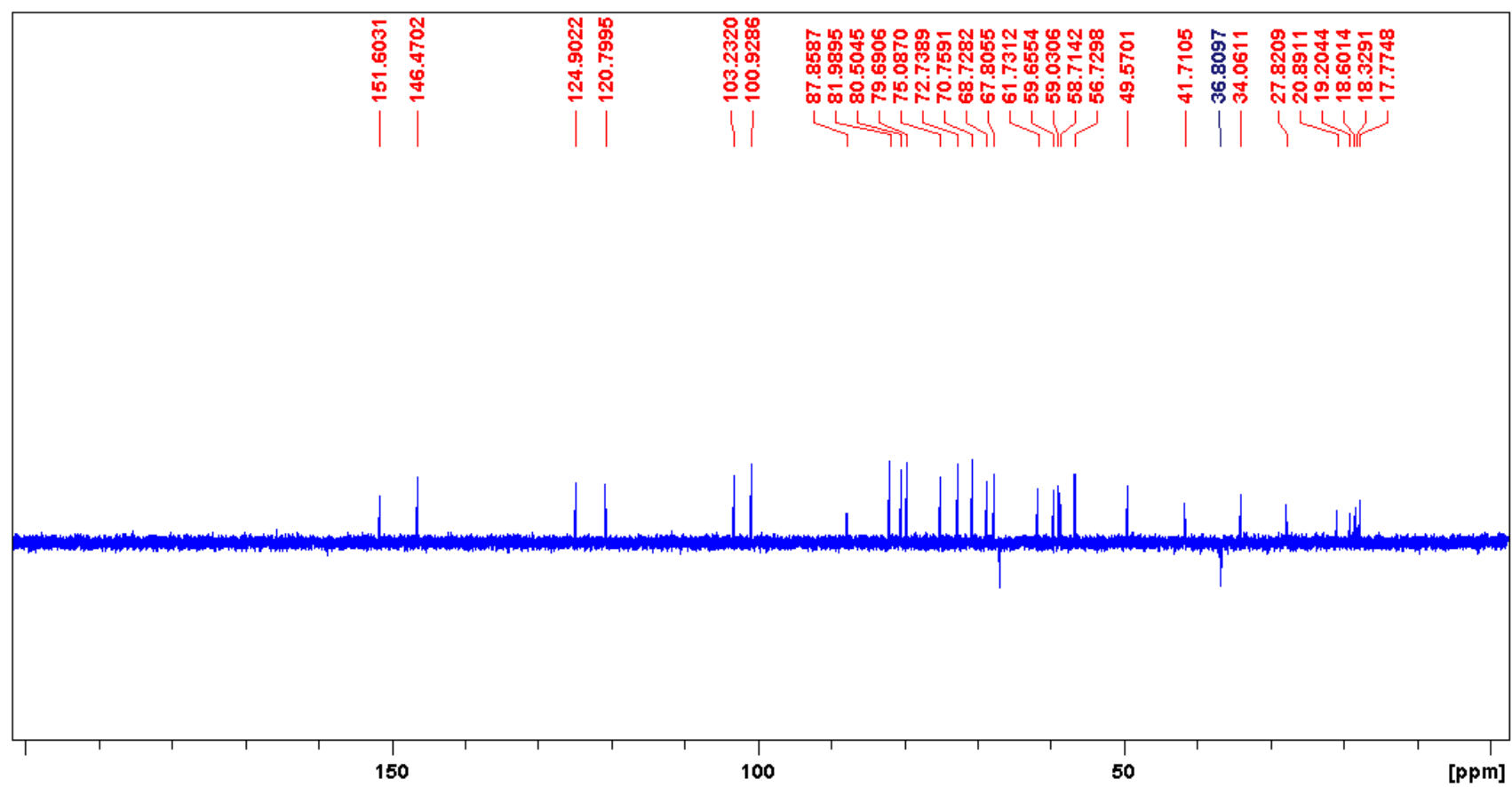

Figure S50. DEPT135 (100 MHz, $\left.\mathrm{CDCl}_{3}\right)$ spectrum for chalcomycin (8) 


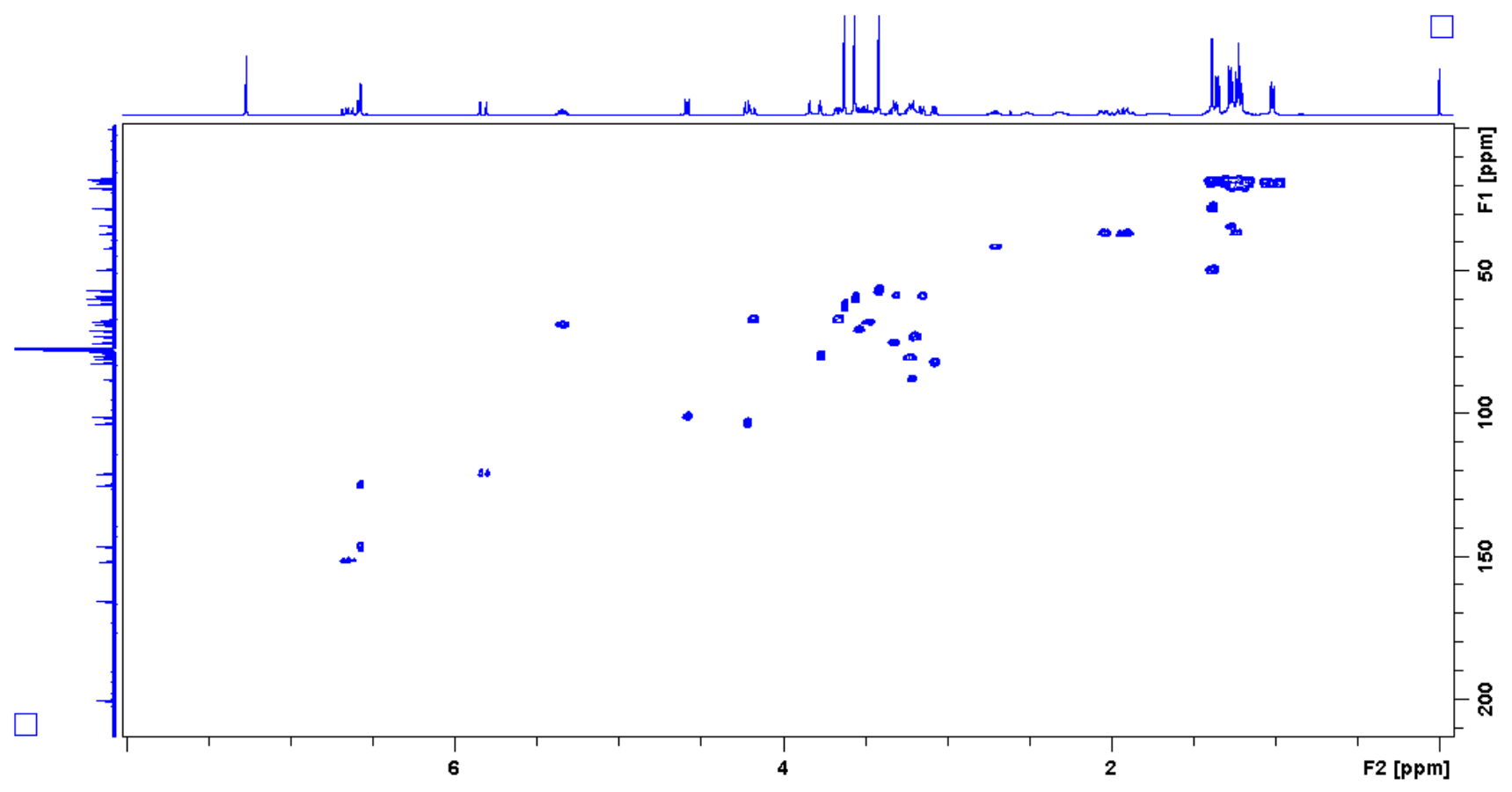

Figure S51. HSQC $\left(\mathrm{CDCl}_{3}\right)$ spectrum for chalcomycin (8)

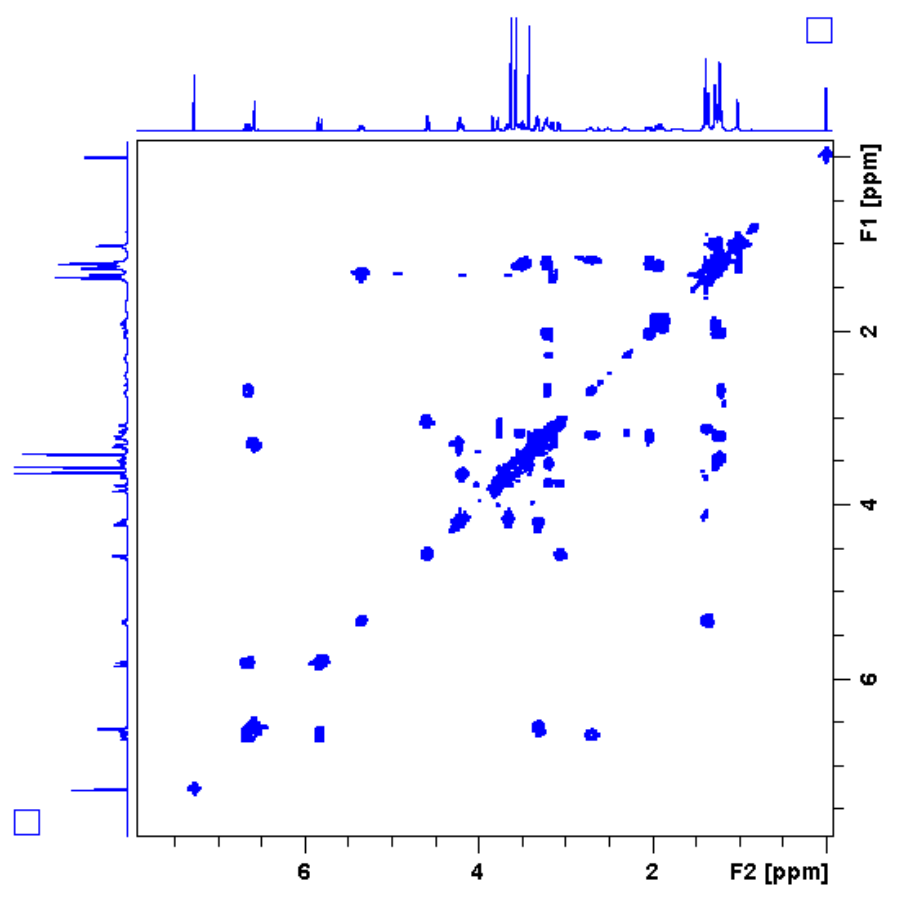

Figure S52. ${ }^{1} \mathrm{H}-{ }^{1} \mathrm{H} \operatorname{COSY}\left(\mathrm{CDCl}_{3}\right)$ spectrum for chalcomycin $(\mathbf{8})$ 


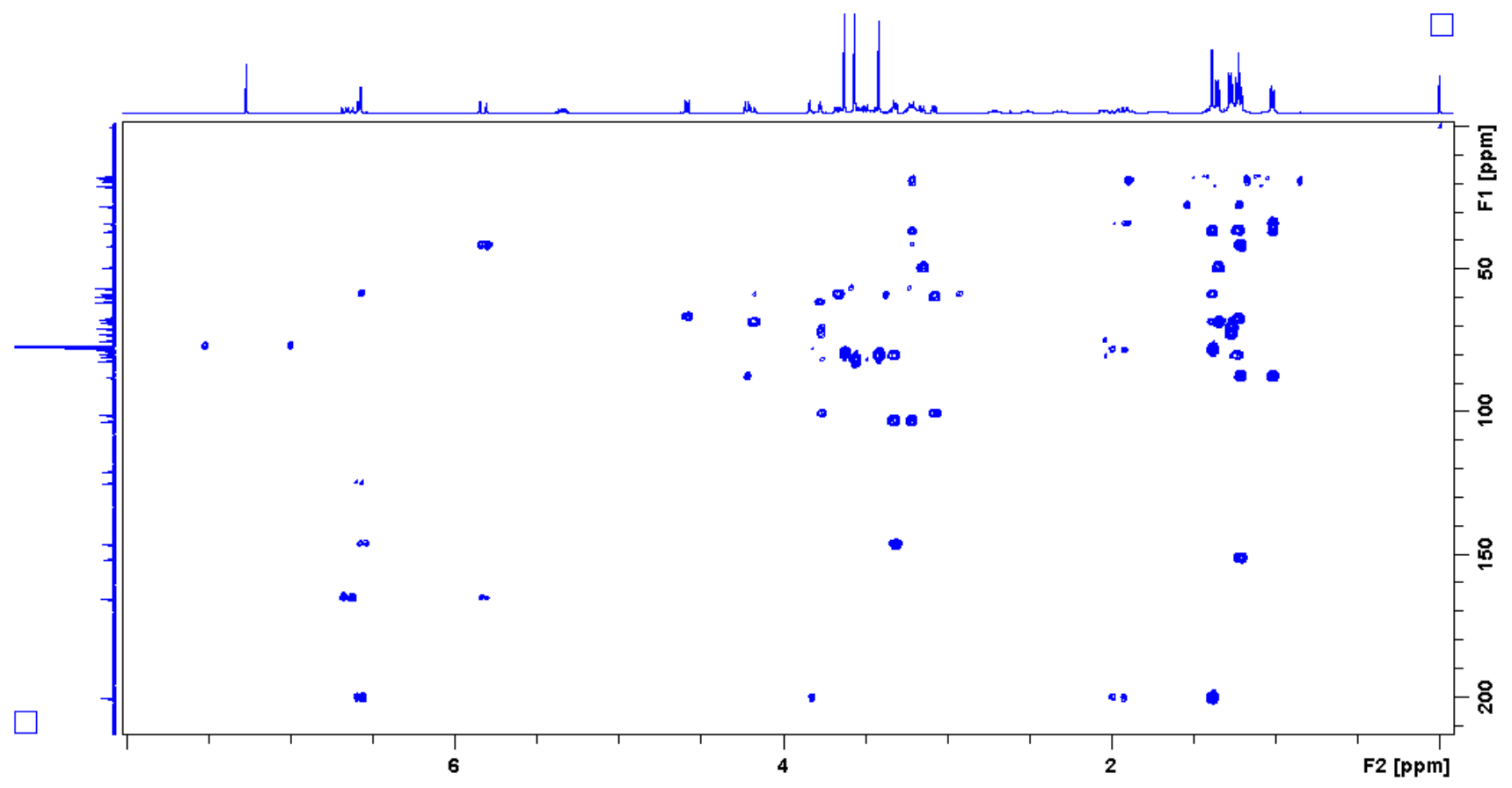

Figure S53. $\mathrm{HMBC}\left(\mathrm{CDCl}_{3}\right)$ spectrum for chalcomycin $(\mathbf{8})$ 